Article

\title{
Performance Analysis of Single-Step Localization Method Based on Matrix Eigen-Perturbation Theory with System Errors
}

\author{
Tianzhu Qin *, Bin Ba and Daming Wang \\ PLA Strategic Support Force Information Engineering University, Zhengzhou 450001, China; \\ xidianbabin@163.com (B.B.); wdm_wangdaming@163.com (D.W.) \\ * Correspondence: skypillar@outlook.com; Tel.: + 86-188-3896-7979
}

Received: 16 January 2019; Accepted: 11 February 2019; Published: 19 February 2019

check for updates

\begin{abstract}
Direct position determination (DPD) is a novel technique in passive localization field recently, receiving superior localization performance compared with the conventional two-step method. The DPD estimator using Doppler shifts is first proposed by Weiss, but it is not suitable for antenna arrays. Additionally, the performance analysis of this method with system errors is absent. This study discusses the single-step localization problem based on moving arrays and exhibits the performance analysis via matrix eigen-perturbation theory with system errors. First, the DPD method using angle of arrival and Doppler shifts is introduced. Then, by adding the eigenvalue perturbations to the estimated Hermitian matrix, the asymptotic linear formulation of localization errors is derived. Consequently, the mean square error of the DPD method is available. Finally, Cramér-Rao bound without system errors is presented, providing a benchmark for the best localization precision and revealing the influence of system errors on the localization precision. Simulation results demonstrate the theoretical analysis in this study.
\end{abstract}

Keywords: direct position determination; array signal processing; Doppler shifts; matrix eigen-perturbation theory; system errors; Cramér-Rao bound

\section{Introduction}

Transmitter localization has attracted significant attention in wireless communication systems. Generally, the conventional localization approach employs a two-step processing. In the first step, the measurement parameters (e.g., direction of arrival (DOA) [1], time of arrival (TOA) [2], time difference of arrival (TDOA) [3], Doppler shifts [4-6], and frequency difference of arrival (FDOA) [7]) are extracted from the received signal. In the second step, the transmitter position is determined by these estimated parameters via maximum likelihood criterion [8] of subspace data fusion criterion [9]. Although the conventional two-step localization method has been extensively investigated in social location system, it cannot achieve high localization accuracy. Indeed, it is suboptimal, because the intermediate parameters are extracted independently by each receiver station, with ignoring the constraint that all observations must relate to the same geolocation of the emitter. Recently, direct position determination (DPD), which exploits the intrinsic constraint and determines the source position from the received signals in a single step, is regarded as an emerging technology in the field of localization. Compared with the conventional two-step approach, the DPD technique [10-18] receives superior localization performance especially under low signal-to-noise (SNR) conditions.

The DPD algorithms have been intensively investigated in recent years. Weiss first proposes the DPD method for narrowband source based on Doppler shifts in Reference [10]. Then, Tirer and Weiss investigate a high-resolution method based on minimum variance distortonless response 
(MVDR) without the knowledge of the number of emitters in Reference [11]. To locate the wideband random emitter, the DPD approach based on time delay and Doppler shifts is developed by Weiss [12]. Additionally, a fast DPD method for known wideband signal waveforms is developed by [13]. It should be emphasized that each receiver in above methods is equipped with only one antenna. As a result, the DOA information is not fully utilized. In Reference [14], a DPD approach using antenna arrays is first investigated, demonstrating the effectiveness of the DOA information on improving performance. Based on the work of [14], the DPD methods applied in special conditions are developed. A DPD estimator for a novel localization architecture, called "Multiple Transponders and Multiple Receivers for Multiple Emitters Positioning System" is proposed [15]. DPD approaches are further designed for sources with special properties such as constant modulus [16], orthogonal frequency division multiplexing [17], and cyclostationary [18]. It is easily observed that the DOA information is conducive to the improvement of positioning accuracy. Moreover, note that all results in Reference [10-18] reveal that the direct positioning method has a superior localization performance than that of the conventional two-step method, especially under low SNRs.

In wireless localization scenarios, system errors (i.e., the uncertainty of the receiver position and velocity) often occur. Obviously, the localization performance is deteriorated by environment noise and system errors in this condition. It is well known that the intermediate parameter estimation is sensitive to system errors. When system errors exist, Cherchar [19] and Vincent [20] give statistical performance analyses of the DOA estimation based on SDF and ML criterions, respectively; Hu [21] deduces the localization performance analysis using TDOA and FDOA; and Hari [22] provides an effect of spatial smoothing on the performance analysis of subspace methods. However, the above analyses are only served for the conventional two-step location estimator. It can be predicted that system errors also affect the localization precision of the DPD estimator. The performance of the DPD approach is available in Reference [23-25], but it is only useful for known signal waveforms. Following the work of [14], Wang presents the performance analysis for unknown signal waveforms in presence of array model errors in Reference [26]. Furthermore, Tirer provides the performance analysis of a high-resolution DPD method based on MVDR in Reference [27]. However, the results in Reference $[27,28]$ could not be applied in moving arrays application. Consequently, in the presence of system errors, there is a strong demand for the performance analysis of single-step method for unknown signal waveforms with moving arrays.

Because the single-step approach in Reference [10] plays a fundamental role in this field, we make related improvement and analysis based on it. Following the requirements of current situations, this paper extends the DPD estimator in Reference [10] to moving arrays application, and exhibits the performance analysis via matrix eigen-perturbation theory with system errors. First of all, the signal model is reconstructed by using Doppler and DOA information. Then, since the solution in DPD method is expressed by finding the maximum eigenvalue of the Hermitian matrix in the cost function, system errors can be shown as eigenvalue perturbations on this Hermitian matrix. Based on matrix eigen-perturbation results, which express the perturbations as an additive noise on the Hertmitian matrix, a theoretical analysis is presented. Moreover, the expression of the mean square error (MSE) of direct localization with system errors is provided. Finally, the Cramér-Rao bound (CRB) formulation for the single-step method is also derived, which gives a benchmark for the best localization accuracy for any estimator. Note that the localization errors of the DPD estimator can asymptotically reach the associated CRB in Reference [10]. It is worth mentioning that the CRB is in absence of system errors, which plays a reference to measure the precision loss resulted from system errors.

The rest of this paper is organized as follows. Section 2 lists the notations used in this paper. Section 3 constructs the signal model and formulates the problem. Section 4 discusses the extension of Weiss's method. Section 5 gives the statistically performance analysis of the DPD estimator with system errors. Section 6 presents several numerical simulations to verify the theoretical analysis. Finally, Section 7 draws the conclusions. 


\section{Notations}

In this section, some mathematical notation explanations that will be used through this paper are listed in Table 1.

Table 1. Mathematics notation explanation

\begin{tabular}{cc}
\hline Notation & Explanation \\
\hline$[\cdot]^{\mathrm{T}}$ & transpose \\
\hline$[\cdot]^{\mathrm{H}}$ & conjugate transpose \\
\hline$(R)$ & real part \\
\hline.$(I)$ & imaginary part \\
\hline $\operatorname{diag}\{\cdot\}$ & diagonal matrix with diagonal entries \\
\hline $\operatorname{blkdiag}\{\cdot\}$ & diagonal matrix with diagonal matrices \\
\hline$\otimes$ & Kronecker product \\
\hline $\boldsymbol{I}_{n}$ & $n \times n$ identity matrix \\
\hline $0_{n}$ & $n \times n$ matrix with zero \\
\hline
\end{tabular}

\section{Signal Model}

We consider $L$ moving receivers and a stationary emitter in this scenario. These receivers intercept the received signal at $K$ short intervals along their trajectory. To introduce the DPD signal model, two assumptions are made.

Assumption 1. Let $\boldsymbol{o}_{l, k}$ and $\boldsymbol{v}_{l, k}$ denote the coordinate and velocity vector of the $l$ th receiver at the $k$ th interception interval. For easy expression, let $\boldsymbol{p}_{l, k}=\left[\boldsymbol{o}_{l, k}^{\mathrm{T}}, \boldsymbol{v}_{l, k}^{\mathrm{T}}\right]^{\mathrm{T}}$ denote the system parameter of the $l$ th receiver. The observation is quiye short, thereby these two vectors are unchanged at each interception interval. Furthermore, let $z$ denote the emitter position.

Assumption 2. The signal bandwidth is small compared to the inverse of the propagation time among receivers (i.e., $B<1 / \tau_{\max }$, where $\tau_{\max }$ denotes the maximal propagation time among the receivers). Consequently, the observer's spatial separation receives a limitation for a given signal bandwidth.

After being sampled at $t=n T_{s}$, the complex signals $\widetilde{\boldsymbol{y}}_{l, k}(n)$ observed by the $l$ th receiver at the $k$ th interception interval is expressed as

$$
\widetilde{\boldsymbol{y}}_{l, k}(n)=b_{l, k} \boldsymbol{a}\left(\boldsymbol{z}, \boldsymbol{o}_{l, k}\right) s_{k}(n) e^{j 2 \pi f_{l, k}}+\boldsymbol{n}_{l, k}(n) n=1, \ldots, N
$$

for $l=1, \ldots, L$ and $k=1, \ldots, K$, where $N$ denotes the number of sample points at each interval. During the $k$ th interception interval, $b_{l, k}$ and $\boldsymbol{a}\left(\boldsymbol{z}, \boldsymbol{o}_{l, k}\right)$ are the channel attenuation and the steering vector between the emitter and the $l$ th receiver, $s_{k}(n)$ is the unknown complex signal envelope of the emitter, $\boldsymbol{n}_{l, k}(n)$ denotes the Gaussian noise vector, and $f_{l, k}$ is the Doppler frequency observed by the $l$ th receiver is expressed by [10]

$$
f_{l, k}=\Delta f_{k}+f_{c} \mu_{l, k}\left(z, p_{l, k}\right)
$$

where $\Delta f_{k}$ is the unknown transmitted frequency, $f_{c}$ is the nominal frequency, and $\mu_{l, k}\left(z, p_{l, k}\right)$ is shown as

$$
\mu_{l, k}\left(z, p_{l, k}\right)=\frac{1}{c} \frac{\boldsymbol{v}_{l, k}^{\mathrm{T}}\left(z-\boldsymbol{o}_{l, k}\right)}{\left\|z-\boldsymbol{o}_{l, k}\right\|} .
$$

here $c$ is the signal speed. Then, (1) can be expressed by a vector form as

$$
\widetilde{\boldsymbol{y}}_{l, k}=b_{l, k} \boldsymbol{C}\left(\boldsymbol{z}, \boldsymbol{p}_{l, k}\right) \boldsymbol{B}_{k} \boldsymbol{s}_{k}+\boldsymbol{n}_{l, k}=\boldsymbol{y}_{l, k}+\boldsymbol{n}_{l, k}
$$


where

$$
\begin{aligned}
& \widetilde{\boldsymbol{y}}_{l, k}=\left[\widetilde{\boldsymbol{y}}_{l, k}^{\mathrm{T}}(1), \widetilde{\boldsymbol{y}}_{l, k}^{\mathrm{T}}(2), \ldots, \widetilde{\boldsymbol{y}}_{l, k}^{\mathrm{T}}(N)\right]^{\mathrm{T}} \\
& s_{k}=\left[s_{k}(1), s_{k}(2), \ldots, s_{k}(N)\right]^{\mathrm{T}} \\
& \boldsymbol{n}_{l, k}=\left[\boldsymbol{n}_{l, k}^{\mathrm{T}}(1), \boldsymbol{n}_{l, k}^{\mathrm{T}}(2), \ldots, \boldsymbol{n}_{l, k}^{\mathrm{T}}(N)\right]^{\mathrm{T}} \\
& C\left(z, p_{l, k}\right)=a\left(z, o_{l, k}\right) \otimes A\left(z, p_{l, k}\right) \\
& \boldsymbol{a}\left(\boldsymbol{z}, \boldsymbol{o}_{l, k}\right)=\left[1, e^{j 2 \pi \frac{d}{\lambda} \sin \theta_{l, k}}, \ldots, e^{j 2 \pi \frac{d}{\lambda}(M-1) \sin \theta_{l, k}}\right]^{\mathrm{T}} \\
& \boldsymbol{A}\left(\boldsymbol{z}, \boldsymbol{p}_{l, k}\right)=\operatorname{diag}\left\{\exp \left(j 2 \pi f_{c} \mu\left(\boldsymbol{z}, \boldsymbol{p}_{l, k}\right) \tilde{\boldsymbol{N}} T_{s}\right)\right\} \\
& \boldsymbol{B}_{k}=\operatorname{diag}\left\{\exp \left(j 2 \pi \Delta f_{k} \widetilde{N} T_{s}\right)\right\}
\end{aligned}
$$

with $\widetilde{N}=[1,2, \ldots, N]^{\mathrm{T}}$. Note that $\lambda$ denotes signal wavelength.

\section{Improvement on Previous Work}

This section discusses the DPD methods, which locate the emitter directly through the raw data. Weiss [10] first proposed a ML-based DPD method using Doppler shifts. However, when array sensors are adopted in receivers, angle information should be used to enhance localization performance. Hence, we extend Weiss's method through the combination of angle and Doppler. The likelihood function for $\widetilde{y}$ can be formulated by

$$
L(\zeta)=\frac{1}{\left(\pi \sigma^{2}\right)^{L K M N}} \exp \left(-\frac{1}{\sigma^{2}} \sum_{k=1}^{K} \sum_{l=1}^{L}\left\|\widetilde{\boldsymbol{y}}_{l, k}-b_{l, k} \boldsymbol{C}\left(\boldsymbol{z}, \boldsymbol{p}_{l, k}\right) \boldsymbol{B}_{k} \boldsymbol{s}_{k}\right\|_{2}^{2}\right)
$$

$\zeta$ denotes all unknown parameters, where

$$
\boldsymbol{\theta}=\left[\boldsymbol{b}^{(R) \mathrm{T}}, \boldsymbol{b}^{(I) \mathrm{T}}, \boldsymbol{s}^{(R) \mathrm{T}}, \boldsymbol{s}^{(I) \mathrm{T}}, \Delta \boldsymbol{f}^{\mathrm{T}}, \boldsymbol{z}^{\mathrm{T}}\right]^{\mathrm{T}}
$$

here, $\boldsymbol{b}=\left[\boldsymbol{b}_{1}^{\mathrm{T}}, \boldsymbol{b}_{2}^{\mathrm{T}}, \ldots, \boldsymbol{b}_{K}^{\mathrm{T}}\right]^{\mathrm{T}}$ with $\boldsymbol{b}_{k}=\left[b_{1, k}, b_{2, k}, \ldots, b_{L, k}\right]^{\mathrm{T}}, \boldsymbol{s}=\left[\boldsymbol{s}_{1}^{\mathrm{T}}, \boldsymbol{s}_{2}^{\mathrm{T}}, \ldots, \boldsymbol{s}_{K}^{\mathrm{T}}\right]^{\mathrm{T}}, \Delta \boldsymbol{f}=$ $\left[\Delta f_{1}, \Delta f_{2}, \ldots, \Delta f_{K}\right]^{\mathrm{T}}$. The associated logarithmic likelihood function can be written as

$$
L_{L n}(\boldsymbol{\zeta})=-L K M N \ln \pi \sigma^{2}-\frac{1}{\sigma^{2}} \sum_{k=1}^{K} \sum_{l=1}^{L}\left\|\widetilde{\boldsymbol{y}}_{l, k}-b_{l, k} \boldsymbol{C}\left(\boldsymbol{z}, \boldsymbol{p}_{l, k}\right) \boldsymbol{B}_{k} \boldsymbol{s}_{k}\right\|_{2}^{2}
$$

Therefore, the estimation of noise power $\sigma^{2}$ is

$$
\hat{\sigma}^{2}=\frac{1}{L K M N} \sum_{k=1}^{K} \sum_{l=1}^{L}\left\|\widetilde{y}_{l, k}-b_{l, k} C\left(z, p_{l, k}\right) B_{k} s_{k}\right\|_{2}^{2}
$$

By substituting (9) into (8), the estimation of parameter $\zeta$ can be determined by

$$
\left\{\hat{b}_{l, k}, \hat{\boldsymbol{s}}_{k}, \Delta \hat{f}_{k}, \hat{z}\right\}=\operatorname{argmin} \sum_{k=1}^{K} \sum_{l=1}^{L}\left\|\widetilde{y}_{l, k}-b_{l, k} \boldsymbol{C}\left(\boldsymbol{z}, \boldsymbol{p}_{l, k}\right) \boldsymbol{B}_{k} \boldsymbol{s}_{k}\right\|_{2}^{2}
$$

Next, the optimization solution of (10) is provided. First, by minimizing the above expression, $\hat{b}_{l, k}$ is estimated by [14]

$$
\hat{b}_{l, k}=\frac{\left(\boldsymbol{C}\left(\boldsymbol{z}, \boldsymbol{p}_{l, k}\right) \boldsymbol{B}_{k} \boldsymbol{s}_{k}\right)^{\mathrm{H}} \widetilde{\boldsymbol{y}}_{l, k}}{\left(\boldsymbol{C}\left(\boldsymbol{z}, \boldsymbol{p}_{l, k}\right) \boldsymbol{B}_{k} \boldsymbol{s}_{k}\right)^{\mathrm{H}}\left(\boldsymbol{C}\left(\boldsymbol{z}, \boldsymbol{p}_{l, k}\right) \boldsymbol{B}_{k} \boldsymbol{s}_{k}\right)}=\frac{1}{M}\left(\boldsymbol{C}\left(\boldsymbol{z}, \boldsymbol{p}_{l, k}\right) \boldsymbol{B}_{k} \boldsymbol{s}_{k}\right)^{\mathrm{H}} \widetilde{\boldsymbol{y}}_{l, k}
$$


Then, after applying (11) to (10) and eliminating the constant part, (10) can be written as

$$
\left\{\hat{\boldsymbol{s}}_{k}, \Delta \hat{f}_{k}, \hat{z}\right\}=\operatorname{argmax} \sum_{k=1}^{K} \omega_{k}^{\mathrm{H}} \boldsymbol{D}_{k}\left(\boldsymbol{z}, \boldsymbol{p}_{k}, \boldsymbol{n}_{k}\right) \boldsymbol{D}_{k}^{\mathrm{H}}\left(\boldsymbol{z}, \boldsymbol{p}_{k}, \boldsymbol{n}_{k}\right) \omega_{k}
$$

where

$$
\left\{\begin{aligned}
\omega_{k}=\boldsymbol{B}_{k} \boldsymbol{s}_{k} & \\
\boldsymbol{D}_{k}\left(\boldsymbol{z}, \boldsymbol{p}_{k}, \boldsymbol{n}_{k}\right) & =\left[\boldsymbol{C}^{\mathrm{H}}\left(\boldsymbol{z}, \boldsymbol{p}_{1, k}\right) \widetilde{\boldsymbol{y}}_{1, k}, \boldsymbol{C}^{\mathrm{H}}\left(\boldsymbol{z}, \boldsymbol{p}_{2, k}\right) \widetilde{\boldsymbol{y}}_{2, k}, \ldots, \boldsymbol{C}^{\mathrm{H}}\left(\boldsymbol{z}, \boldsymbol{p}_{L, k}\right) \widetilde{\boldsymbol{y}}_{L, k}\right] \\
& =\overline{\boldsymbol{C}}\left(\boldsymbol{z}, \boldsymbol{p}_{k}\right)\left(\boldsymbol{Y}_{k}+\boldsymbol{N}_{k}\right)
\end{aligned}\right.
$$

with

$$
\left\{\begin{array}{l}
\overline{\boldsymbol{C}}\left(\boldsymbol{z}, \boldsymbol{p}_{k}\right)=\left[\boldsymbol{C}^{\mathrm{H}}\left(\boldsymbol{z}, \boldsymbol{p}_{1, k}\right), \boldsymbol{C}^{\mathrm{H}}\left(\boldsymbol{z}, \boldsymbol{p}_{2, k}\right), \ldots, \boldsymbol{C}^{\mathrm{H}}\left(\boldsymbol{z}, \boldsymbol{p}_{L, k}\right)\right] \\
\boldsymbol{Y}_{k}=\operatorname{blkdiag}\left\{\boldsymbol{y}_{1, k}, \boldsymbol{y}_{2, k}, \ldots, \boldsymbol{y}_{L, k}\right\} \\
\boldsymbol{N}_{k}=\operatorname{blkdiag}\left\{\boldsymbol{n}_{1, k}, \boldsymbol{n}_{2, k}, \ldots, \boldsymbol{n}_{L, k}\right\}
\end{array}\right.
$$

Note that $\omega_{k}$ is unknown to receivers. The maximization of (12) is solved by choosing the vector $\omega_{k}$ as the eigenvector associated with the largest eigenvalue of matrix $\boldsymbol{D}_{k}\left(\boldsymbol{z}, \boldsymbol{p}_{k}, \boldsymbol{n}_{k}\right) \boldsymbol{D}_{k}^{\mathrm{H}}\left(\boldsymbol{z}, \boldsymbol{p}_{k}, \boldsymbol{n}_{k}\right)$. Therefore, the optimization problem in (12) respect to $z$ is expressed by

$$
\hat{z}=\operatorname{argmax} \sum_{k=1}^{K} \lambda_{\max }\left\{\boldsymbol{D}_{k}\left(\boldsymbol{z}, \boldsymbol{p}_{k}, \boldsymbol{n}_{k}\right) \boldsymbol{D}_{k}^{\mathrm{H}}\left(\boldsymbol{z}, \boldsymbol{p}_{k}, \boldsymbol{n}_{k}\right)\right\}
$$

where $\lambda_{\max }\{\cdot\}$ denotes the largest eigenvalues of the matrix.

It must be emphasized that the matrices $\boldsymbol{D}_{k}\left(\boldsymbol{z}, \boldsymbol{p}_{k}, \boldsymbol{n}_{k}\right) \boldsymbol{D}_{k}^{\mathrm{H}}\left(\boldsymbol{z}, \boldsymbol{p}_{k}, \boldsymbol{n}_{k}\right)$ and $\boldsymbol{D}_{k}^{\mathrm{H}}\left(\boldsymbol{z}, \boldsymbol{p}_{k}, \boldsymbol{n}_{k}\right) \boldsymbol{D}_{k}\left(\boldsymbol{z}, \boldsymbol{p}_{k}, \boldsymbol{n}_{k}\right)$ share the same nonzero eigenvalues. Generally, the dimension of matrix $\boldsymbol{D}_{k}^{\mathrm{H}}\left(\boldsymbol{z}, \boldsymbol{p}_{k}, \boldsymbol{n}_{k}\right) \boldsymbol{D}_{k}\left(\boldsymbol{z}, \boldsymbol{p}_{k}, \boldsymbol{n}_{k}\right) \in$ $\mathbb{C}^{L \times L}$ is significantly smaller than that of $\boldsymbol{D}_{k}\left(\boldsymbol{z}, \boldsymbol{p}_{k}, \boldsymbol{n}_{k}\right) \boldsymbol{D}_{k}^{\mathrm{H}}\left(\boldsymbol{z}, \boldsymbol{p}_{k}, \boldsymbol{n}_{k}\right) \in \mathbb{C}^{N \times N}$. Hence, to reach for lower computational cost, the estimation of $z$ can be replaced by

$$
\hat{z}=\operatorname{argmax} \sum_{k=1}^{K} \lambda_{\max }\left\{\boldsymbol{D}_{k}^{\mathrm{H}}\left(\boldsymbol{z}, \boldsymbol{p}_{k}, \boldsymbol{n}_{k}\right) \boldsymbol{D}_{k}\left(\boldsymbol{z}, \boldsymbol{p}_{k}, \boldsymbol{n}_{k}\right)\right\}
$$

To fully describe the proposed method, we make a computational complexity analysis. Based on the above derivation, the calculation of $\boldsymbol{D}_{k}^{\mathrm{H}}\left(\boldsymbol{z}, \boldsymbol{p}_{k}, \boldsymbol{n}_{k}\right) \boldsymbol{D}_{k}\left(\boldsymbol{z}, \boldsymbol{p}_{k}, \boldsymbol{n}_{k}\right)$ and grid search in the position set of interest make a major contribution to the computational load. The total number of calculation equals $O\left(L M^{2} N^{3}+(1+M) N^{2}+(1+N) L^{3}+M\right) K N_{p}$, where $N_{p}$ is the number of grid search points in terms of emitter position. Since Weiss's method uses only an antenna at each receiver, the value of $M$ should be 1 . Therefore, the computational load of Weiss's method is $O\left(L N^{3}+2 N^{2}+(1+N) L^{3}\right) K N_{p}$. It is readily observed that the computational complexity of out method is heavier than that of Weiss's method. Even with more computing resources, on the other hand, our method can offer superior performance (see Section 6.2).

\section{Statistical Performance Analysis}

It is well known that the above DPD method can reach asymptotic optimal with precise system parameters. However, system errors (i.e., the position and velocity uncertainties of airplanes or UAVs) often occur in real life, which deteriorate the localization precision of the above DPD method greatly. For this reason, in this section, the perturbation analysis and the MSE of the DPD method in presence of system errors will be discussed.

We assume that the real parameters are defined as $p_{k}(k=1, \ldots, K)$, the observed parameters are written as $\bar{p}_{k}(k=1, \ldots, K)$, and the system errors are expressed by $\widetilde{p}_{k}(k=1, \ldots, K)$. The relation between these parameters is

$$
\widetilde{\boldsymbol{p}}_{k}=\overline{\boldsymbol{p}}_{k}-\boldsymbol{p}_{k} k=1, \ldots, \mathrm{K}
$$


Therefore, the estimation of $z$ in presence of system errors should be determined by

$$
\hat{z}=\operatorname{argmax} \sum_{k=1}^{K} \lambda_{\max }\left\{\boldsymbol{D}_{k}^{\mathrm{H}}\left(\boldsymbol{z}, \overline{\boldsymbol{p}}_{k}, \boldsymbol{n}_{k}\right) \boldsymbol{D}_{k}\left(\boldsymbol{z}, \overline{\boldsymbol{p}}_{k}, \boldsymbol{n}_{k}\right)\right\}
$$

Obviously, the localization performance analysis related to noise $n_{k}(k=1, \ldots, K)$ and system errors $\widetilde{p}_{k}(k=1, \ldots, K)$ should be considered simultaneously. To complete this result analysis, matrix eigen-perturbation theory needs to be applied on (16).

\subsection{Basic Theoretical Analysis Tool}

Note that the key part of Weiss's method is finding the maximal eigenvalue of Hermitian matrix, which is disturbed by other error matrix. Relevant theory can be expressed by:

Proposition 1. Assume that $Q \in \mathbb{C}^{N \times N}$ is a positive semidefinite respect to eigenvalues $\left.\lambda_{n}\right|_{1 \leq n \leq N}$ and unit eigenvectors $\left.\boldsymbol{\alpha}_{n}\right|_{1 \leq n \leq N}$. Moreover, assume that $\boldsymbol{Q}$ is disturbed by a matrix $\widetilde{\boldsymbol{Q}} \in \mathbb{C}^{N \times N}$, hence the perturbed matrix can be written as $\bar{Q}=Q+\widetilde{Q}$. Finally, the relation of the eigenvalues $\left(\left.\bar{\lambda}_{n}\right|_{1 \leq n \leq N}\right)$ of $\bar{Q}$ and $\left.\lambda_{n}\right|_{1 \leq n \leq N}$ is shown as

$$
\bar{\lambda}_{n}=\lambda_{n}+\boldsymbol{\alpha}_{n}^{\mathrm{H}} \widetilde{\boldsymbol{Q}} \boldsymbol{\alpha}_{n}+\boldsymbol{\alpha}_{n}^{\mathrm{H}} \widetilde{\boldsymbol{Q}} \boldsymbol{E}_{n} \widetilde{\boldsymbol{Q}} \boldsymbol{\alpha}_{n}+o\left(\|\widetilde{\boldsymbol{Q}}\|_{2}^{2}\right) n=1, \ldots, N
$$

where $\boldsymbol{E}_{n}=\sum_{\substack{i=1 \\ i \neq n}}^{N}\left(\lambda_{n}-\lambda_{i}\right)^{-1} \boldsymbol{\alpha}_{i} \boldsymbol{\alpha}_{i}^{\mathrm{H}}$. The detailed proof of this proposition can be found in $[25,26]$.

\subsection{Perturbation Analysis on The Cost Function}

As mentioned earlier, our purpose is investigating the relationship between the MSE of the DPD estimator and noise as well as system errors. Herein, we adopt a second-order perturbation analysis to (18), which follows

$$
\boldsymbol{D}_{k}\left(\hat{\boldsymbol{z}}, \overline{\boldsymbol{p}}_{k}, \boldsymbol{n}_{k}\right)=\overline{\boldsymbol{C}}\left(\hat{\boldsymbol{z}}, \overline{\boldsymbol{p}}_{k}\right)\left(\boldsymbol{Y}_{k}+\boldsymbol{N}_{k}\right) \approx \boldsymbol{D}_{k}^{(0)}+\widetilde{\boldsymbol{D}}_{k}^{(1)}+\widetilde{\boldsymbol{D}}_{k}^{(2)}
$$

It is necessary to emphasize that $\boldsymbol{D}_{k}^{(0)}$ is the non-perturbation terms, and $\widetilde{\boldsymbol{D}}_{k}^{(1)}$ as well as $\widetilde{\boldsymbol{D}}_{k}^{(2)}$ denote the first and second-order perturbation terms, respectively. Their expression is specified by

$$
\left\{\begin{array}{l}
\boldsymbol{D}_{k}^{(0)}=\overline{\boldsymbol{C}}\left(\boldsymbol{z}, \boldsymbol{p}_{k}\right) \boldsymbol{Y}_{k} \\
\widetilde{\boldsymbol{D}}_{k}^{(1)}=\sum_{d=1}^{D}\langle\widetilde{\boldsymbol{z}}\rangle_{d} \dot{\overline{\boldsymbol{C}}}_{d}^{(a)}\left(\boldsymbol{z}, \boldsymbol{p}_{k}\right) \boldsymbol{Y}_{k}+\sum_{d=1}^{2 D L}\left\langle\widetilde{\boldsymbol{p}}_{k}\right\rangle_{d} \dot{\overline{\boldsymbol{C}}}_{d}^{(b)}\left(\boldsymbol{z}, \boldsymbol{p}_{k}\right) \boldsymbol{Y}_{k}+\overline{\boldsymbol{C}}\left(\boldsymbol{z}, \boldsymbol{p}_{k}\right) \boldsymbol{N}_{k} \\
\widetilde{\boldsymbol{D}}_{k}^{(2)}=\frac{1}{2} \sum_{d_{1}=1}^{D} \sum_{d_{2}=1}^{D}\langle\widetilde{\boldsymbol{z}}\rangle_{d_{1}}\langle\widetilde{\boldsymbol{z}}\rangle_{d_{2}} \ddot{\overline{\boldsymbol{C}}}_{d_{1} d_{2}}^{(a a)}\left(\boldsymbol{z}, \boldsymbol{p}_{k}\right) \boldsymbol{Y}_{k}+\frac{1}{2} \sum_{d_{1}=1}^{2 D L} \sum_{d_{2}=1}^{2 D L}\left\langle\widetilde{\boldsymbol{p}}_{k}\right\rangle_{d_{1}}\left\langle\widetilde{\boldsymbol{p}}_{k}\right\rangle_{d_{2}} \ddot{\overline{\boldsymbol{C}}}_{d_{1} d_{2}}^{(b b)}\left(\boldsymbol{z}, \boldsymbol{p}_{k}\right) \boldsymbol{Y}_{k}+ \\
\sum_{d_{1}=1}^{D} \sum_{d_{2}=1}^{2 D L}\langle\widetilde{\boldsymbol{z}}\rangle_{d_{1}}\left\langle\widetilde{\boldsymbol{p}}_{k}\right\rangle_{d_{2}} \ddot{\overline{\boldsymbol{C}}}_{d_{1} d_{2}}^{(a b)}\left(\boldsymbol{z}, \boldsymbol{p}_{k}\right) \boldsymbol{Y}_{k}+\sum_{d=1}^{D}\langle\widetilde{\boldsymbol{z}}\rangle_{d} \dot{\overline{\boldsymbol{C}}}_{d}^{(a)}\left(\boldsymbol{z}, \boldsymbol{p}_{k}\right) \boldsymbol{N}_{k}+\sum_{d=1}^{2 D L}\left\langle\widetilde{\boldsymbol{p}}_{k}\right\rangle_{d} \dot{\overline{\boldsymbol{C}}}_{d}^{(b)}\left(\boldsymbol{z}, \boldsymbol{p}_{k}\right) \boldsymbol{N}_{k}
\end{array}\right.
$$

where

$$
\left\{\begin{array}{l}
\dot{\bar{C}}_{d}^{(a)}\left(\boldsymbol{z}, \boldsymbol{p}_{k}\right)=\frac{\partial \overline{\mathcal{C}}\left(z, p_{k}\right)}{\partial\langle z\rangle_{d}}, \dot{\bar{C}}_{d}^{(b)}\left(\boldsymbol{z}, \boldsymbol{p}_{k}\right)=\frac{\partial \overline{\bar{C}}\left(z, p_{k}\right)}{\partial\left\langle\boldsymbol{p}_{k}\right\rangle_{d}}, \ddot{\bar{C}}_{d_{1} d_{2}}^{(a a)}\left(\boldsymbol{z}, \boldsymbol{p}_{k}\right)=\frac{\partial^{2} \overline{\mathcal{C}}\left(\boldsymbol{z}, p_{k}\right)}{\partial\langle z\rangle_{d_{1}} \partial\langle z\rangle_{d_{2}}} \\
\ddot{\bar{C}}_{d_{1} d_{2}}^{(b b)}\left(\boldsymbol{z}, \boldsymbol{p}_{k}\right)=\frac{\partial^{2} \overline{\bar{C}}\left(z, \boldsymbol{p}_{k}\right)}{\partial\left\langle\boldsymbol{p}_{k}\right\rangle_{d_{1}} \partial\left\langle\boldsymbol{p}_{k}\right\rangle_{d_{2}}}, \ddot{\bar{C}}_{d_{1} d_{2}}^{(a b)}\left(\boldsymbol{z}, \boldsymbol{p}_{k}\right)=\frac{\partial^{2} \overline{\bar{C}}\left(z, p_{k}\right)}{\partial\langle z\rangle_{d_{1}} \partial\left\langle\boldsymbol{p}_{k}\right\rangle_{d_{2}}}
\end{array}\right.
$$

The derivation of (21) is exhibited in Appendix A, and the matrices in (22) are listed in Appendix B. 
For easy derivation, we define $\boldsymbol{Q}_{k}\left(\hat{z}, \overline{\boldsymbol{p}}_{k}, \boldsymbol{n}_{k}\right)=\boldsymbol{D}_{k}^{\mathrm{H}}\left(\hat{z}, \overline{\boldsymbol{p}}_{k}, \boldsymbol{n}_{k}\right) \cdot \boldsymbol{D}_{k}\left(\hat{\boldsymbol{z}}, \overline{\boldsymbol{p}}_{k}, \boldsymbol{n}_{k}\right)$. Following the result in (20), the Hermitian matrix $\boldsymbol{Q}_{k}\left(\hat{z}, \bar{p}_{k}, \boldsymbol{n}_{k}\right)$ is approximated by

$$
\boldsymbol{Q}_{k}\left(\hat{z}, \overline{\boldsymbol{p}}_{k}, \boldsymbol{n}_{k}\right) \approx \boldsymbol{Q}_{k}^{(0)}+\widetilde{\boldsymbol{Q}}_{k}^{(1)}+\widetilde{\boldsymbol{Q}}_{k}^{(2)}
$$

where

$$
\left\{\begin{array}{l}
\boldsymbol{Q}_{k}^{(0)}=\boldsymbol{D}_{k}^{(0) \mathrm{H}} \boldsymbol{D}_{k}^{(0)} \\
\widetilde{\boldsymbol{Q}}_{k}^{(1)}=\boldsymbol{D}_{k}^{(0) \mathrm{H}} \widetilde{\boldsymbol{D}}_{k}^{(1)}+\widetilde{\boldsymbol{D}}_{k}^{(1) \mathrm{H}} \boldsymbol{D}_{k}^{(0)} \\
\widetilde{\boldsymbol{Q}}_{k}^{(2)}=\boldsymbol{D}_{k}^{(0) \mathrm{H}} \widetilde{\boldsymbol{D}}_{k}^{(2)}+\widetilde{\boldsymbol{D}}_{k}^{(1) \mathrm{H}} \widetilde{\boldsymbol{D}}_{k}^{(1)}+\widetilde{\boldsymbol{D}}_{k}^{(2) \mathrm{H}} \boldsymbol{D}_{k}^{(0)}
\end{array}\right.
$$

Note that error matrix is defined as

$$
\widetilde{Q}_{k}=Q_{k}\left(\hat{z}, \bar{p}_{k}, \boldsymbol{n}_{k}\right)-Q_{k}^{(0)} \approx \widetilde{\boldsymbol{Q}}_{k}^{(1)}+\widetilde{\boldsymbol{Q}}_{k}^{(2)}
$$

which is obtained by neglecting the high-order error issues.

Assume that $Q_{k}^{(0)}$ is related to eigenvalues $\left.\lambda_{k, l}^{(0)}\right|_{1 \leq l \leq L}$ as well as unit eigenvectors $\left.\boldsymbol{\alpha}_{k, l}^{(0)}\right|_{1 \leq l \leq L}$, and $\boldsymbol{Q}_{k}\left(\hat{\boldsymbol{z}}, \overline{\boldsymbol{p}}_{k}, \boldsymbol{n}_{k}\right)$ is associated with eigenvalues $\left.\bar{\lambda}_{k, l}\right|_{1 \leq l \leq L}$ as well as unit eigenvectors $\left.\overline{\boldsymbol{\alpha}}_{k, l}\right|_{1 \leq l \leq L}$. By following the result in Proposition 1, we obtain

$$
\bar{\lambda}_{k, L}=\lambda_{k, L}^{(0)}+\boldsymbol{\alpha}_{k, L}^{(0) \mathrm{H}} \widetilde{\boldsymbol{Q}}_{k} \boldsymbol{\alpha}_{k, L}^{(0)}+\boldsymbol{\alpha}_{k, L}^{(0) \mathrm{H}} \widetilde{\boldsymbol{Q}}_{k} \boldsymbol{E}_{k, L} \widetilde{\boldsymbol{Q}}_{k} \boldsymbol{\alpha}_{k, L}^{(0)}+o\left(\left\|\widetilde{\boldsymbol{Q}}_{k}\right\|_{2}^{2}\right) k=1, \ldots, K
$$

where $\boldsymbol{E}_{k, L}=\sum_{i=1}^{L}\left(\lambda_{k, L}^{(0)}-\lambda_{k, i}^{(0)}\right)^{-1} \boldsymbol{\alpha}_{k, i}^{(0)} \boldsymbol{\alpha}_{k, i}^{(0) \mathrm{H}}$.

Inserting (25) into (26) leads to

$$
\bar{\lambda}_{k, L} \approx \lambda_{k, L}^{(0)}+\widetilde{\lambda}_{k, L}^{(1)}+\widetilde{\lambda}_{k, L}^{(2)}
$$

where $\tilde{\lambda}_{k, L}^{(1)}$ and $\tilde{\lambda}_{k, L}^{(2)}$ denote the first- and second-order distributed issues, respectively

$$
\left\{\begin{aligned}
\widetilde{\lambda}_{k, L}^{(1)}= & \boldsymbol{\alpha}_{k, L}^{(0) \mathrm{H}} \boldsymbol{D}_{k}^{(0) \mathrm{H}} \widetilde{\boldsymbol{D}}_{k}^{(1)} \boldsymbol{\alpha}_{k, L}^{(0)}+\boldsymbol{\alpha}_{k, L}^{(0) \mathrm{H}} \widetilde{\boldsymbol{D}}_{k}^{(1) \mathrm{H}} \boldsymbol{D}_{k}^{(0)} \boldsymbol{\alpha}_{k, L}^{(0)} \\
\widetilde{\lambda}_{k, L}^{(2)}= & \boldsymbol{\alpha}_{k, L}^{(0) \mathrm{H}} \boldsymbol{D}_{k}^{(0) \mathrm{H}} \widetilde{\boldsymbol{D}}_{k}^{(2)} \boldsymbol{\alpha}_{k, L}^{(0)}+\boldsymbol{\alpha}_{k, L}^{(0) \mathrm{H}} \widetilde{\boldsymbol{D}}_{k}^{(1) \mathrm{H}} \widetilde{\boldsymbol{D}}_{k}^{(1)} \boldsymbol{\alpha}_{k, L}^{(0)}+\boldsymbol{\alpha}_{k, L}^{(0) \mathrm{H}} \widetilde{\boldsymbol{D}}_{k}^{(2) \mathrm{H}} \boldsymbol{D}_{k}^{(0)} \boldsymbol{\alpha}_{k, L}^{(0)}+ \\
& \boldsymbol{\alpha}_{k, L}^{(0) \mathrm{H}} \boldsymbol{D}_{k}^{(0) \mathrm{H}} \widetilde{\boldsymbol{D}}_{k}^{(1)} \boldsymbol{E}_{k, L} \boldsymbol{D}_{k}^{(0) \mathrm{H}} \widetilde{\boldsymbol{D}}_{k}^{(1)} \boldsymbol{\alpha}_{k, L}^{(0)}+\boldsymbol{\alpha}_{k, L}^{(0) \mathrm{H}} \widetilde{\boldsymbol{D}}_{k}^{(1) \mathrm{H}} \boldsymbol{D}_{k}^{(0)} \boldsymbol{E}_{k, L} \boldsymbol{D}_{k}^{(0) \mathrm{H}} \widetilde{\boldsymbol{D}}_{k}^{(1)} \boldsymbol{\alpha}_{k, L}^{(0)}+ \\
& \boldsymbol{\alpha}_{k, L}^{(0) \mathrm{H}} \boldsymbol{D}_{k}^{(0) \mathrm{H}} \widetilde{\boldsymbol{D}}_{k}^{(1)} \boldsymbol{E}_{k, L} \widetilde{\boldsymbol{D}}_{k}^{(1) \mathrm{H}} \boldsymbol{D}_{k}^{(0)} \boldsymbol{\alpha}_{k, L}^{(0)}+\boldsymbol{\alpha}_{k, L}^{(0) \mathrm{H}} \widetilde{\boldsymbol{D}}_{k}^{(1) \mathrm{H}} \boldsymbol{D}_{k}^{(0)} \boldsymbol{E}_{k, L} \widetilde{\boldsymbol{D}}_{k}^{(1) \mathrm{H}} \boldsymbol{D}_{k}^{(0)} \boldsymbol{\alpha}_{k, L}^{(0)}
\end{aligned}\right.
$$

Define $J_{\text {cost }}(\hat{z}, \overline{\boldsymbol{p}}, \boldsymbol{n})=\sum_{k=1}^{K} \lambda_{\max }\left\{\boldsymbol{D}_{k}^{\mathrm{H}}\left(\boldsymbol{z}, \overline{\boldsymbol{p}}_{k}, \boldsymbol{n}_{k}\right) \boldsymbol{D}_{k}\left(\boldsymbol{z}, \overline{\boldsymbol{p}}_{k}, \boldsymbol{n}_{k}\right)\right\}$ and apply (27) in (18). Then, $J_{\operatorname{cost}}(\hat{z}, \bar{p}, \boldsymbol{n})$ can be approximated by

$$
J_{\text {cost }}(\hat{z}, \bar{p}, \boldsymbol{n}) \approx J_{\text {cost }}^{(0)}+\widetilde{J}_{\text {cost }}^{(1)}+\widetilde{J}_{\text {cost }}^{(2)}
$$

where

$$
J_{\cos t}^{(0)}=\sum_{k=1}^{K} \lambda_{k, L^{\prime}}^{(0)} \widetilde{J}_{\cos t}^{(1)}=\sum_{k=1}^{K} \widetilde{\lambda}_{k, L^{\prime}}^{(1)} \widetilde{J}_{\cos t}^{(2)}=\sum_{k=1}^{K} \widetilde{\lambda}_{k, L}^{(2)}
$$

more specially, $\widetilde{J}_{\text {cost }}^{(1)}$ is written as

$$
\begin{aligned}
\widetilde{J}_{\text {cost }}^{(1)} & =\sum_{k=1}^{K} \boldsymbol{\alpha}_{k, L}^{(0) \mathrm{H}} \boldsymbol{D}_{k}^{(0) \mathrm{H}} \widetilde{D}_{k}^{(1)} \boldsymbol{\alpha}_{k, L}^{(0)}+\sum_{k=1}^{K} \boldsymbol{\alpha}_{k, L}^{(0) \mathrm{H}} \widetilde{D}_{k}^{(1) \mathrm{H}} \boldsymbol{D}_{k}^{(0)} \boldsymbol{\alpha}_{k, L}^{(0)} \\
& =\sum_{k=1}^{K} f_{1 k}^{\mathrm{H}} \widetilde{z}+\sum_{k=1}^{K} f_{2 k}^{\mathrm{H}} \widetilde{p}_{k}+\sum_{k=1}^{K} f_{3 k}^{\mathrm{H}} \widetilde{n}_{k}
\end{aligned}
$$


where

$$
\left\{\begin{array}{l}
\widetilde{\boldsymbol{n}}_{k}=\left[\boldsymbol{n}_{k}^{\mathrm{T}} \boldsymbol{n}_{k}^{\mathrm{H}}\right]^{\mathrm{T}} \\
f_{n k}=\boldsymbol{F}_{n k}^{(a) \mathrm{H}}\left(\boldsymbol{\alpha}_{k, L}^{(0)}\right) \boldsymbol{D}_{k}^{(0)} \boldsymbol{\alpha}_{k, L}^{(0)}+\boldsymbol{F}_{n k}^{(b) \mathrm{H}}\left(\boldsymbol{D}_{k}^{(0)} \boldsymbol{\alpha}_{k, L}^{(0)}\right) \boldsymbol{\alpha}_{k, L}^{(0)} n=1,2,3
\end{array}\right.
$$

with

$$
\begin{aligned}
& \left\{\begin{array}{l}
\boldsymbol{F}_{1 k}^{(a)}(\boldsymbol{q})=\frac{\partial\left(\overline{\boldsymbol{C}}\left(z, \boldsymbol{p}_{k}\right) \boldsymbol{Y}_{k} \boldsymbol{q}\right)}{\partial z^{\mathrm{T}}}, \boldsymbol{F}_{2 k}^{(a)}(\boldsymbol{q})=\frac{\partial\left(\overline{\boldsymbol{C}}\left(z, \boldsymbol{p}_{k}\right) \boldsymbol{Y}_{k} \boldsymbol{q}\right)}{\partial \boldsymbol{p}_{k}^{\mathrm{T}}}, \\
\boldsymbol{F}_{3 k}^{(a)}(\boldsymbol{q})=\overline{\boldsymbol{C}}\left(\boldsymbol{z}, \boldsymbol{p}_{k}\right)\left(\operatorname{diag}\{\boldsymbol{q}\} \otimes \boldsymbol{I}_{M N}\right) \boldsymbol{\Pi}_{1}
\end{array}\right. \\
& \left\{\begin{array}{l}
\boldsymbol{F}_{1 k}^{(b)}(\boldsymbol{q})=\frac{\partial\left(\chi_{k}^{\mathrm{H}} \overline{\boldsymbol{C}}^{\mathrm{H}}\left(\boldsymbol{z}, \boldsymbol{p}_{k}\right) \boldsymbol{q}\right)}{\partial \boldsymbol{z}^{\mathrm{T}}}, \boldsymbol{F}_{2 k}^{(b)}(\boldsymbol{q})=\frac{\partial\left(\boldsymbol{\gamma}_{k}^{\mathrm{H}} \overline{\boldsymbol{C}}^{\mathrm{H}}\left(\boldsymbol{z}, \boldsymbol{p}_{k}\right) \boldsymbol{q}\right)}{\partial \boldsymbol{p}_{k}^{\mathrm{T}}}, \\
\boldsymbol{F}_{3 k}^{(b)}(\boldsymbol{q})=\left(\boldsymbol{I}_{L} \otimes \boldsymbol{q}^{\mathrm{T}}\right) \overline{\overline{\boldsymbol{C}}}\left(\boldsymbol{z}, \boldsymbol{p}_{k}\right) \boldsymbol{\Pi}_{2}
\end{array}\right.
\end{aligned}
$$

where

$$
\left\{\begin{array}{l}
\boldsymbol{\Pi}_{1}=\left[\boldsymbol{I}_{M N L} \boldsymbol{0}_{M N L}\right] \\
\boldsymbol{\Pi}_{2}=\left[0_{M N L} \boldsymbol{I}_{M N L}\right] \\
\overline{\bar{C}}\left(\boldsymbol{z}, \boldsymbol{p}_{k}\right)=\operatorname{blkdiag}\left\{\boldsymbol{C}^{\mathrm{T}}\left(\boldsymbol{z}, \boldsymbol{p}_{1, k}\right), \boldsymbol{C}^{\mathrm{T}}\left(\boldsymbol{z}, \boldsymbol{p}_{2, k}\right), \ldots, \boldsymbol{C}^{\mathrm{T}}\left(\boldsymbol{z}, \boldsymbol{p}_{L, k}\right)\right\}
\end{array}\right.
$$

The detailed derivation of (31) to (34) can be seen in Appendix C.

Furthermore, $\widetilde{J}_{\text {cost }}^{(2)}$ can be formulated as

$$
\begin{aligned}
\widetilde{J}_{\text {cost }}^{(2)}= & \sum_{k=1}^{K} \boldsymbol{\alpha}_{k, L}^{(0) \mathrm{H}} \boldsymbol{D}_{k}^{(0) \mathrm{H}} \widetilde{D}_{k}^{(2)} \boldsymbol{\alpha}_{k, L}^{(0)}+\sum_{k=1}^{K} \boldsymbol{\alpha}_{k, L}^{(0) \mathrm{H}} \widetilde{D}_{k}^{(2) \mathrm{H}} \boldsymbol{D}_{k}^{(0)} \boldsymbol{\alpha}_{k, L}^{(0)}+\sum_{k=1}^{K} \boldsymbol{\alpha}_{k, L}^{(0) \mathrm{H}} \widetilde{D}_{k}^{(1) \mathrm{H}} \widetilde{D}_{k}^{(1)} \boldsymbol{\alpha}_{k, L}^{(0)}+ \\
& \sum_{k=1}^{K} \boldsymbol{\alpha}_{k, L}^{(0) \mathrm{H}} \boldsymbol{D}_{k}^{(0) \mathrm{H}} \widetilde{D}_{k}^{(1)} \boldsymbol{E}_{k, L} \boldsymbol{D}_{k}^{(0) \mathrm{H}} \widetilde{D}_{k}^{(1)} \boldsymbol{\alpha}_{k, L}^{(0)}+\sum_{k=1}^{K} \boldsymbol{\alpha}_{k, L}^{(0) \mathrm{H}} \widetilde{D}_{k}^{(1) \mathrm{H}} \boldsymbol{D}_{k}^{(0)} \boldsymbol{E}_{k, L} \boldsymbol{D}_{k}^{(0) \mathrm{H}} \widetilde{D}_{k}^{(1)} \boldsymbol{\alpha}_{k, L}^{(0)}+ \\
& \sum_{k=1}^{K} \boldsymbol{\alpha}_{k, L}^{(0) \mathrm{H}} \boldsymbol{D}_{k}^{(0) \mathrm{H}} \widetilde{D}_{k}^{(1)} \boldsymbol{E}_{k, L} \widetilde{D}_{k}^{(1) \mathrm{H}} \boldsymbol{D}_{k}^{(0)} \boldsymbol{\alpha}_{k, L}^{(0)}+\sum_{k=1}^{K} \boldsymbol{\alpha}_{k, L}^{(0) \mathrm{H}} \widetilde{D}_{k}^{(1) \mathrm{H}} \boldsymbol{D}_{k}^{(0)} \boldsymbol{E}_{k, L} \widetilde{D}_{k}^{(1) \mathrm{H}} \boldsymbol{D}_{k}^{(0)} \boldsymbol{\alpha}_{k, L}^{(0)} \\
= & \sum_{k=1}^{K} \widetilde{z}^{\mathrm{T}} \boldsymbol{\xi}_{1 k} \widetilde{z}+\sum_{k=1}^{K} \widetilde{p}_{k}^{\mathrm{T}} \boldsymbol{\xi}_{2 k} \widetilde{p}_{k}+\sum_{k=1}^{K} \widetilde{z}^{\mathrm{T}} \boldsymbol{\xi}_{3 k} \widetilde{p}_{k}+\sum_{k=1}^{K} \widetilde{z}^{\mathrm{T}} \boldsymbol{\xi}_{4 k} \widetilde{n}_{k}+\sum_{k=1}^{K} \widetilde{p}_{k}^{\mathrm{T}} \boldsymbol{\xi}_{5 k} \widetilde{n}_{k}+\sum_{k=1}^{K} \widetilde{n}_{k}^{\mathrm{H}} \boldsymbol{\xi}_{6 k} \widetilde{n}_{k}
\end{aligned}
$$

where

$$
\begin{aligned}
& \boldsymbol{\xi}_{i k}=\Sigma_{i k}^{(a)}\left(\boldsymbol{\alpha}_{k, L^{\prime}}^{(0)} \boldsymbol{I}_{N}, \boldsymbol{\alpha}_{k, L}^{(0)}\right)+\boldsymbol{\Sigma}_{i k}^{(a)}\left(\boldsymbol{\alpha}_{k, L^{\prime}}^{(0)} \boldsymbol{D}_{k}^{(0)} \boldsymbol{E}_{k, L} \boldsymbol{D}_{k}^{(0) \mathrm{H}}, \boldsymbol{\alpha}_{k, L}^{(0)}\right)+ \\
& \boldsymbol{\Sigma}_{i k}^{(b)}\left(\boldsymbol{D}_{k}^{(0)} \boldsymbol{\alpha}_{k, L}^{(0)}, \boldsymbol{E}_{k, L}, \boldsymbol{D}_{k}^{(0)} \boldsymbol{\alpha}_{k, L}^{(0)}\right)+\boldsymbol{\Sigma}_{i k}^{(c)}\left(\boldsymbol{D}_{k}^{(0)} \boldsymbol{\alpha}_{k, L}^{(0)}, \boldsymbol{E}_{k, L} \boldsymbol{D}_{k}^{(0) \mathrm{H}}, \boldsymbol{\alpha}_{k, L}^{(0)}\right)+ \\
& \boldsymbol{\Sigma}_{i k}^{(c) *}\left(\boldsymbol{D}_{k}^{(0)} \boldsymbol{\alpha}_{k, L}^{(0)}, \boldsymbol{E}_{k, L} \boldsymbol{D}_{k}^{(0) \mathrm{H}}, \boldsymbol{\alpha}_{k, L}^{(0)}\right)+\boldsymbol{\Sigma}_{i k}^{(d)}\left(\boldsymbol{D}_{k}^{(0)} \boldsymbol{\alpha}_{k, L}^{(0)}, \boldsymbol{\alpha}_{k, L}^{(0)}\right)+ \\
& \boldsymbol{\Sigma}_{i k}^{(d) *}\left(\boldsymbol{D}_{k}^{(0)} \boldsymbol{\alpha}_{k, L}^{(0)}, \boldsymbol{\alpha}_{k, L}^{(0)}\right)(1 \leq i \leq 3) \\
& \boldsymbol{\xi}_{j k}=\boldsymbol{\Sigma}_{j k}^{(a)}\left(\boldsymbol{\alpha}_{k, L}^{(0)}, \boldsymbol{I}_{N}, \boldsymbol{\alpha}_{k, L}^{(0)}\right)+\boldsymbol{\Sigma}_{j k}^{(a)}\left(\boldsymbol{\alpha}_{k, L}^{(0)}, \boldsymbol{D}_{k}^{(0)} \boldsymbol{E}_{k, L} \boldsymbol{D}_{k}^{(0) \mathrm{H}}, \boldsymbol{\alpha}_{k, L}^{(0)}\right)+ \\
& \boldsymbol{\Sigma}_{j k}^{(b)}\left(\boldsymbol{D}_{k}^{(0)} \boldsymbol{\alpha}_{k, L}^{(0)}, \boldsymbol{E}_{k, L}, \boldsymbol{D}_{k}^{(0)} \boldsymbol{\alpha}_{k, L}^{(0)}\right)+\boldsymbol{\Sigma}_{j k}^{(c)}\left(\boldsymbol{D}_{k}^{(0)} \boldsymbol{\alpha}_{k, L}^{(0)}, \boldsymbol{E}_{k, L} \boldsymbol{D}_{k}^{(0) \mathrm{H}}, \boldsymbol{\alpha}_{k, L}^{(0)}\right)+ \\
& \Sigma_{j k}^{(c) *}\left(\boldsymbol{D}_{k}^{(0)} \boldsymbol{\alpha}_{k, L}^{(0)}, \boldsymbol{E}_{k, L}^{\mathrm{H}} \boldsymbol{D}_{k}^{(0) \mathrm{H}}, \boldsymbol{\alpha}_{k, L}^{(0)}\right) \boldsymbol{\Pi}_{3}+\boldsymbol{\Sigma}_{j k}^{(d)}\left(\boldsymbol{D}_{k}^{(0)} \boldsymbol{\alpha}_{k, L}^{(0)}, \boldsymbol{\alpha}_{k, L}^{(0)}\right)+ \\
& \boldsymbol{\Sigma}_{j k}^{(d) *}\left(\boldsymbol{D}_{k}^{(0)} \boldsymbol{\alpha}_{k, L^{\prime}}^{(0)} \boldsymbol{\alpha}_{k, L}^{(0)}\right) \boldsymbol{\Pi}_{3}(j=4,5) \\
& \xi_{6 k}=\Sigma_{6 k}^{(a)}\left(\boldsymbol{\alpha}_{k, L}^{(0)}, \boldsymbol{I}_{N}, \boldsymbol{\alpha}_{k, L}^{(0)}\right)+\boldsymbol{\Sigma}_{6 k}^{(a)}\left(\boldsymbol{\alpha}_{k, L}^{(0)}, \boldsymbol{D}_{k}^{(0)} \boldsymbol{E}_{k, L} \boldsymbol{D}_{k}^{(0) \mathrm{H}}, \boldsymbol{\alpha}_{k, L}^{(0)}\right)+ \\
& \boldsymbol{\Sigma}_{6 k}^{(b)}\left(\boldsymbol{D}_{k}^{(0)} \boldsymbol{\alpha}_{k, L}^{(0)}, \boldsymbol{E}_{k, L}, \boldsymbol{D}_{k}^{(0)} \boldsymbol{\alpha}_{k, L}^{(0)}\right)+\boldsymbol{\Sigma}_{6 k}^{(c)}\left(\boldsymbol{D}_{k}^{(0)} \boldsymbol{\alpha}_{k, L^{\prime}}^{(0)} \boldsymbol{E}_{k, L} \boldsymbol{D}_{k}^{(0) \mathrm{H}}, \boldsymbol{\alpha}_{k, L}^{(0)}\right)+ \\
& \Sigma_{6 k}^{(c) \mathrm{H}}\left(\boldsymbol{D}_{k}^{(0)} \boldsymbol{\alpha}_{k, L}^{(0)}, \boldsymbol{E}_{k, L}^{\mathrm{H}} \boldsymbol{D}_{k}^{(0) \mathrm{H}}, \boldsymbol{\alpha}_{k, L}^{(0)}\right)
\end{aligned}
$$

with

$$
\left\{\begin{array}{l}
\boldsymbol{\Sigma}_{1 k}^{(a)}\left(\boldsymbol{q}_{1}, \boldsymbol{\Phi}, \boldsymbol{q}_{2}\right)=\boldsymbol{F}_{1 k}^{(a) \mathrm{H}}\left(\boldsymbol{q}_{1}\right) \boldsymbol{\Phi} \boldsymbol{F}_{1 k}^{(a)}\left(\boldsymbol{q}_{2}\right), \boldsymbol{\Sigma}_{2 k}^{(a)}\left(\boldsymbol{q}_{1}, \boldsymbol{\Phi}, \boldsymbol{q}_{2}\right)=\boldsymbol{F}_{2 k}^{(a) \mathrm{H}}\left(\boldsymbol{q}_{1}\right) \boldsymbol{\Phi} \boldsymbol{F}_{2 k}^{(a)}\left(\boldsymbol{q}_{2}\right) \\
\boldsymbol{\Sigma}_{3 k}^{(a)}\left(\boldsymbol{q}_{1}, \boldsymbol{\Phi}, \boldsymbol{q}_{2}\right)=\boldsymbol{F}_{1 k}^{(a) \mathrm{H}}\left(\boldsymbol{q}_{1}\right) \boldsymbol{\Phi} \boldsymbol{F}_{2 k}^{(a)}\left(\boldsymbol{q}_{2}\right)+\boldsymbol{F}_{1 k}^{(a) \mathrm{T}}\left(\boldsymbol{q}_{2}\right) \boldsymbol{\Phi}^{\mathrm{T}} \boldsymbol{F}_{2 k}^{(a) *}\left(\boldsymbol{q}_{1}\right) \\
\boldsymbol{\Sigma}_{4 k}^{(a)}\left(\boldsymbol{q}_{1}, \boldsymbol{\Phi}, \boldsymbol{q}_{2}\right)=\boldsymbol{F}_{1 k}^{(a) \mathrm{H}}\left(\boldsymbol{q}_{1}\right) \boldsymbol{\Phi} \boldsymbol{F}_{3 k}^{(a)}\left(\boldsymbol{q}_{2}\right)+\boldsymbol{F}_{1 k}^{(a) \mathrm{T}}\left(\boldsymbol{q}_{2}\right) \boldsymbol{\Phi}^{\mathrm{T}} \boldsymbol{F}_{3 k}^{(a) *}\left(\boldsymbol{q}_{1}\right) \boldsymbol{\Pi}_{3} \\
\boldsymbol{\Sigma}_{5 k}^{(a)}\left(\boldsymbol{q}_{1}, \boldsymbol{\Phi}, \boldsymbol{q}_{2}\right)=\boldsymbol{F}_{2 k}^{(a) \mathrm{H}}\left(\boldsymbol{q}_{1}\right) \boldsymbol{\Phi} \boldsymbol{F}_{3 k}^{(a)}\left(\boldsymbol{q}_{2}\right)+\boldsymbol{F}_{2 k}^{(a) \mathrm{T}}\left(\boldsymbol{q}_{2}\right) \boldsymbol{\Phi}^{\mathrm{T}} \boldsymbol{F}_{3 k}^{(a) *}\left(\boldsymbol{q}_{1}\right) \boldsymbol{\Pi}_{3} \\
\boldsymbol{\Sigma}_{6 k}^{(a)}\left(\boldsymbol{q}_{1}, \boldsymbol{\Phi}, \boldsymbol{q}_{2}\right)=\boldsymbol{F}_{3 k}^{(a) \mathrm{H}}\left(\boldsymbol{q}_{1}\right) \boldsymbol{\Phi} \boldsymbol{F}_{3 k}^{(a)}\left(\boldsymbol{q}_{2}\right)
\end{array}\right.
$$




$$
\begin{aligned}
& \left\{\begin{array}{l}
\boldsymbol{\Sigma}_{1 k}^{(b)}\left(\boldsymbol{q}_{1}, \boldsymbol{\Phi}, \boldsymbol{q}_{2}\right)=\boldsymbol{F}_{1 k}^{(b) \mathrm{H}}\left(\boldsymbol{q}_{1}\right) \boldsymbol{\Phi} \boldsymbol{F}_{1 k}^{(b)}\left(\boldsymbol{q}_{2}\right), \boldsymbol{\Sigma}_{2 k}^{(b)}\left(\boldsymbol{q}_{1}, \boldsymbol{\Phi}, \boldsymbol{q}_{2}\right)=\boldsymbol{F}_{2 k}^{(b) \mathrm{H}}\left(\boldsymbol{q}_{1}\right) \boldsymbol{\Phi} \boldsymbol{F}_{2 k}^{(b)}\left(\boldsymbol{q}_{2}\right) \\
\boldsymbol{\Sigma}_{3 k}^{(b)}\left(\boldsymbol{q}_{1}, \boldsymbol{\Phi}, \boldsymbol{q}_{2}\right)=\boldsymbol{F}_{1 k}^{(b) \mathrm{H}}\left(\boldsymbol{q}_{1}\right) \boldsymbol{\Phi} \boldsymbol{F}_{2 k}^{(b)}\left(\boldsymbol{q}_{2}\right)+\boldsymbol{F}_{1 k}^{(b) \mathrm{T}}\left(\boldsymbol{q}_{2}\right) \boldsymbol{\Phi}^{\mathrm{T}} \boldsymbol{F}_{2 k}^{(b) *}\left(\boldsymbol{q}_{1}\right) \\
\boldsymbol{\Sigma}_{4 k}^{(b)}\left(\boldsymbol{q}_{1}, \boldsymbol{\Phi}, \boldsymbol{q}_{2}\right)=\boldsymbol{F}_{1 k}^{(b) \mathrm{H}}\left(\boldsymbol{q}_{1}\right) \boldsymbol{\Phi} \boldsymbol{F}_{3 k}^{(b)}\left(\boldsymbol{q}_{2}\right)+\boldsymbol{F}_{1 k}^{(b) \mathrm{T}}\left(\boldsymbol{q}_{2}\right) \boldsymbol{\Phi}^{\mathrm{T}} \boldsymbol{F}_{3 k}^{(b) *}\left(\boldsymbol{q}_{1}\right) \boldsymbol{\Pi}_{3} \\
\boldsymbol{\Sigma}_{5 k}^{(b)}\left(\boldsymbol{q}_{1}, \boldsymbol{\Phi}, \boldsymbol{q}_{2}\right)=\boldsymbol{F}_{2 k}^{(b) \mathrm{H}}\left(\boldsymbol{q}_{1}\right) \boldsymbol{\Phi} \boldsymbol{F}_{3 k}^{(b)}\left(\boldsymbol{q}_{2}\right)+\boldsymbol{F}_{2 k}^{(b) \mathrm{T}}\left(\boldsymbol{q}_{2}\right) \boldsymbol{\Phi}^{\mathrm{T}} \boldsymbol{F}_{3 k}^{(b) *}\left(\boldsymbol{q}_{1}\right) \boldsymbol{\Pi}_{3} \\
\boldsymbol{\Sigma}_{6 k}^{(b)}\left(\boldsymbol{q}_{1}, \boldsymbol{\Phi}, \boldsymbol{q}_{2}\right)=\boldsymbol{F}_{3 k}^{(b) \mathrm{H}}\left(\boldsymbol{q}_{1}\right) \boldsymbol{\Phi} \boldsymbol{F}_{3 k}^{(b)}\left(\boldsymbol{q}_{2}\right)
\end{array}\right. \\
& \int \Sigma_{1 k}^{(c)}\left(\boldsymbol{q}_{1}, \boldsymbol{\Phi}, \boldsymbol{q}_{2}\right)=\boldsymbol{F}_{1 k}^{(b) \mathrm{H}}\left(\boldsymbol{q}_{1}\right) \boldsymbol{\Phi} \boldsymbol{F}_{1 k}^{(a)}\left(\boldsymbol{q}_{2}\right), \boldsymbol{\Sigma}_{2 k}^{(c)}\left(\boldsymbol{q}_{1}, \boldsymbol{\Phi}, \boldsymbol{q}_{2}\right)=\boldsymbol{F}_{2 k}^{(b) \mathrm{H}}\left(\boldsymbol{q}_{1}\right) \boldsymbol{\Phi} \boldsymbol{F}_{2 k}^{(a)}\left(\boldsymbol{q}_{2}\right) \\
& \Sigma_{3 k}^{(c)}\left(\boldsymbol{q}_{1}, \boldsymbol{\Phi}, \boldsymbol{q}_{2}\right)=\boldsymbol{F}_{1 k}^{(b) \mathrm{H}}\left(\boldsymbol{q}_{1}\right) \boldsymbol{\Phi} \boldsymbol{F}_{2 k}^{(a)}\left(\boldsymbol{q}_{2}\right)+\boldsymbol{F}_{1 k}^{(a) \mathrm{T}}\left(\boldsymbol{q}_{2}\right) \boldsymbol{\Phi}^{\mathrm{T}} \boldsymbol{F}_{2 k}^{(b) *}\left(\boldsymbol{q}_{1}\right) \\
& \left\{\boldsymbol{\Sigma}_{4 k}^{(c)}\left(\boldsymbol{q}_{1}, \boldsymbol{\Phi}, \boldsymbol{q}_{2}\right)=\boldsymbol{F}_{1 k}^{(b) \mathrm{H}}\left(\boldsymbol{q}_{1}\right) \boldsymbol{\Phi} \boldsymbol{F}_{3 k}^{(a)}\left(\boldsymbol{q}_{2}\right)+\boldsymbol{F}_{1 k}^{(a) \mathrm{T}}\left(\boldsymbol{q}_{2}\right) \boldsymbol{\Phi}^{\mathrm{T}} \boldsymbol{F}_{3 k}^{(b) *}\left(\boldsymbol{q}_{1}\right) \boldsymbol{\Pi}_{3}\right. \\
& \boldsymbol{\Sigma}_{5 k}^{(c)}\left(\boldsymbol{q}_{1}, \boldsymbol{\Phi}, \boldsymbol{q}_{2}\right)=\boldsymbol{F}_{2 k}^{(b) \mathrm{H}}\left(\boldsymbol{q}_{1}\right) \boldsymbol{\Phi} \boldsymbol{F}_{3 k}^{(a)}\left(\boldsymbol{q}_{2}\right)+\boldsymbol{F}_{2 k}^{(a) \mathrm{T}}\left(\boldsymbol{q}_{2}\right) \boldsymbol{\Phi}^{\mathrm{T}} \boldsymbol{F}_{3 k}^{(b) *}\left(\boldsymbol{q}_{1}\right) \boldsymbol{\Pi}_{3} \\
& \Sigma_{6 k}^{(c)}\left(\boldsymbol{q}_{1}, \boldsymbol{\Phi}, \boldsymbol{q}_{2}\right)=\boldsymbol{F}_{3 k}^{(b) \mathrm{H}}\left(\boldsymbol{q}_{1}\right) \boldsymbol{\Phi} \boldsymbol{F}_{3 k}^{(a)}\left(\boldsymbol{q}_{2}\right) \\
& \left\{\begin{array}{l}
\boldsymbol{\Sigma}_{1 k}^{(d)}\left(\boldsymbol{q}_{1}, \boldsymbol{q}_{2}\right)=\frac{1}{2} \frac{\partial^{2}\left(\boldsymbol{q}_{1}^{\mathrm{H}} \overline{\boldsymbol{C}}\left(z, \boldsymbol{p}_{k}\right) \boldsymbol{y}_{k} \boldsymbol{q}_{2}\right)}{\partial z \partial z^{\mathrm{T}}}, \boldsymbol{\Sigma}_{2 k}^{(d)}\left(\boldsymbol{q}_{1}, \boldsymbol{q}_{2}\right)=\frac{1}{2} \frac{\partial^{2}\left(\boldsymbol{q}_{1}^{\mathrm{H}} \overline{\boldsymbol{C}}\left(z, \boldsymbol{p}_{k}\right) \boldsymbol{x}_{k} \boldsymbol{q}_{2}\right)}{\partial \boldsymbol{p}_{k} \partial \boldsymbol{p}_{k}^{\mathrm{T}}} \\
\boldsymbol{\Sigma}_{3 k}^{(d)}\left(\boldsymbol{q}_{1}, \boldsymbol{q}_{2}\right)=\frac{\partial^{2}\left(\boldsymbol{q}_{1}^{\mathrm{H}} \overline{\boldsymbol{C}}\left(z, \boldsymbol{p}_{k}\right) \boldsymbol{\gamma}_{k} \boldsymbol{q}_{2}\right)}{\partial z \partial \boldsymbol{p}_{k}^{\mathrm{T}}}, \boldsymbol{\Sigma}_{4 k}^{(d)}\left(\boldsymbol{q}_{1}, \boldsymbol{q}_{2}\right)=\left(\frac{\partial\left(\overline{\boldsymbol{c}}^{\mathrm{H}}\left(\boldsymbol{z}, \boldsymbol{p}_{k}\right) \boldsymbol{q}_{1}\right)}{\partial z^{\mathrm{T}}}\right)^{\mathrm{H}} \cdot\left(\operatorname{diag}\left\{\boldsymbol{q}_{2}\right\} \otimes \boldsymbol{I}_{M N}\right) \boldsymbol{\Pi}_{1} \\
\boldsymbol{\Sigma}_{5 k}^{(d)}\left(\boldsymbol{q}_{1}, \boldsymbol{q}_{2}\right)=\left(\frac{\partial\left(\overline{\boldsymbol{c}}^{\mathrm{H}}\left(z, \boldsymbol{p}_{k}\right) \boldsymbol{q}_{1}\right)}{\partial \boldsymbol{p}_{k}^{\mathrm{T}}}\right)^{\mathrm{H}} \cdot\left(\operatorname{diag}\left\{\boldsymbol{q}_{2}\right\} \otimes \boldsymbol{I}_{M N}\right) \boldsymbol{\Pi}_{1}
\end{array}\right.
\end{aligned}
$$

The detailed derivation is exhibited in Appendix D.

In sight of the above analysis, as a result, the second-order approximation of $J_{\operatorname{cost}}(\hat{z}, \bar{p}, n)$ can be drawn as

$$
\begin{aligned}
J_{\text {cost }}(\hat{z}, \bar{p}, \boldsymbol{n}) \approx & J_{\text {cost }}^{(0)}+\sum_{k=1}^{K} f_{1 k}^{\mathrm{H}} \widetilde{z}+\sum_{k=1}^{K} f_{2 k}^{\mathrm{H}} \widetilde{p}_{k}+\sum_{k=1}^{K} f_{3 k}^{\mathrm{H}} \widetilde{n}_{k} \\
& +\sum_{k=1}^{K} \widetilde{z}^{\mathrm{T}} \boldsymbol{\xi}_{1 k} \widetilde{z}+\sum_{k=1}^{K} \widetilde{p}_{k}^{\mathrm{T}} \boldsymbol{\xi}_{2 k} \widetilde{p}_{k}+\sum_{k=1}^{K} \widetilde{z}^{\mathrm{T}} \boldsymbol{\xi}_{3 k} \widetilde{p}_{k} \\
& +\sum_{k=1}^{K} \widetilde{z}^{\mathrm{T}} \boldsymbol{\xi}_{4 k} \widetilde{n}_{k}+\sum_{k=1}^{K} \boldsymbol{p}_{k}^{\mathrm{T}} \boldsymbol{\xi}_{5 k} \widetilde{n}_{k}+\sum_{k=1}^{K} \widetilde{n}_{k}^{\mathrm{H}} \boldsymbol{\xi}_{6 k} \widetilde{n}_{k}
\end{aligned}
$$

Note that $\left.f_{n k}\right|_{1 \leq n \leq 3}$ can act as the gradient vector, and $\left.\xi_{j k}\right|_{1 \leq j \leq 6}$ can form the Hessian matrix, respectively. It is easily found that the single-step localization errors is linearly associated with the environment noise and system errors. Furthermore, the MSE of DPD estimator is presented in the next subsection.

\subsection{MSE of The Single-Step Method with System Errors}

Following the analysis presented above, it can be easily obtained that

$$
\left\{\begin{array}{l}
\frac{\partial J_{\text {cost }}^{(0)}}{\partial z}=0 \\
\frac{\partial \operatorname{cost}_{\text {cost }}(\hat{z}, \bar{p}, n)}{\partial \hat{z}}=0
\end{array}\right.
$$

Then, via combining the first expression in (44) with (43), we have

$$
\frac{\partial J_{\text {cost }}^{(0)}}{\partial z}=\sum_{k=1}^{K} f_{1 k}^{*}=0
$$

Through the second equality in (44), we imply

$$
\widetilde{\boldsymbol{z}}=\underset{\boldsymbol{q}}{\operatorname{argmax}}\left(\sum_{k=1}^{K} f_{1 k}^{\mathrm{H}} \boldsymbol{q}+\sum_{k=1}^{K} \boldsymbol{q}^{\mathrm{T}} \boldsymbol{\xi}_{1 k} \boldsymbol{q}+\sum_{k=1}^{K} \boldsymbol{q}^{\mathrm{T}} \boldsymbol{\xi}_{3 k} \widetilde{p}_{k}+\sum_{k=1}^{K} \boldsymbol{q}^{\mathrm{T}} \boldsymbol{\xi}_{4 k} \widetilde{n}_{k}\right)
$$


moreover, (46) can be specified by

$$
\begin{aligned}
\widetilde{z} & =-\frac{1}{2}\left(\sum_{k=1}^{K} \xi_{1 k}\right)^{-1}\left(\sum_{k=1}^{K} \xi_{3 k} \widetilde{p}_{k}+\sum_{k=1}^{K} \xi_{4 k} \widetilde{n}_{k}+\sum_{k=1}^{K} f_{1 k}^{*}\right) \\
& =-\frac{1}{2}\left(\sum_{k=1}^{K} \xi_{1 k}\right)^{-1}\left(\sum_{k=1}^{K} \xi_{4 k} \widetilde{n}_{k}\right)-\frac{1}{2}\left(\sum_{k=1}^{K} \xi_{1 k}\right)^{-1}\left(\sum_{k=1}^{K} \xi_{3 k} \widetilde{p}_{k}\right)
\end{aligned}
$$

It is readily observed that the localization error parameter $\widetilde{z}$ is composed of two terms. The first formulation in (47) is associated with the environment noise, which is shown as

$$
\widetilde{z}_{1}=-\frac{1}{2}\left(\sum_{k=1}^{K} \xi_{1 k}\right)^{-1}\left(\sum_{k=1}^{K} \xi_{4 k} \widetilde{n}_{k}\right)
$$

The second equality in (47) is corresponding to the system errors, which is exhibited as

$$
\widetilde{z}_{2}=-\frac{1}{2}\left(\sum_{k=1}^{K} \xi_{1 k}\right)^{-1}\left(\sum_{k=1}^{K} \xi_{3 k} \widetilde{p}_{k}\right)
$$

To perfect the analysis, we make a statistical assumption that the system error vectors $\left.\widetilde{p}_{k}\right|_{1 \leq k \leq K}$ obey zero-mean with covariance matrix $\left.\Omega_{k}\right|_{1 \leq k \leq K}$. As a result, we have the location error covariance matrices

$$
\begin{aligned}
\boldsymbol{R}=\mathrm{E}\left[\widetilde{\boldsymbol{z}} \widetilde{\boldsymbol{z}}^{\mathrm{T}}\right]= & \frac{\sigma^{2}}{4}\left(\sum_{k=1}^{K} \xi_{1 k}\right)^{-1}\left(\sum_{k=1}^{K} \xi_{4 k} \xi_{4 k}^{\mathrm{H}}\right)\left(\sum_{k=1}^{K} \xi_{1 k}^{\mathrm{H}}\right)^{-1}+ \\
& \frac{1}{4}\left(\sum_{k=1}^{K} \xi_{1 k}\right)^{-1}\left(\sum_{k=1}^{K} \xi_{3 k} \Omega_{k} \xi_{3 k}^{\mathrm{H}}\right)\left(\sum_{k=1}^{K} \xi_{1 k}^{\mathrm{H}}\right)^{-1}
\end{aligned}
$$

Note that the first part in (50) is related to environment noise and the second part in (50) is attached by system errors. It should be emphasized that trace $\{\boldsymbol{R}\}$ can represent the MSE of the single-step approach in presence of two kinds of disturbance issues.

To better exhibit the analysis process, we summarize it as Algorithm 1 as follows.

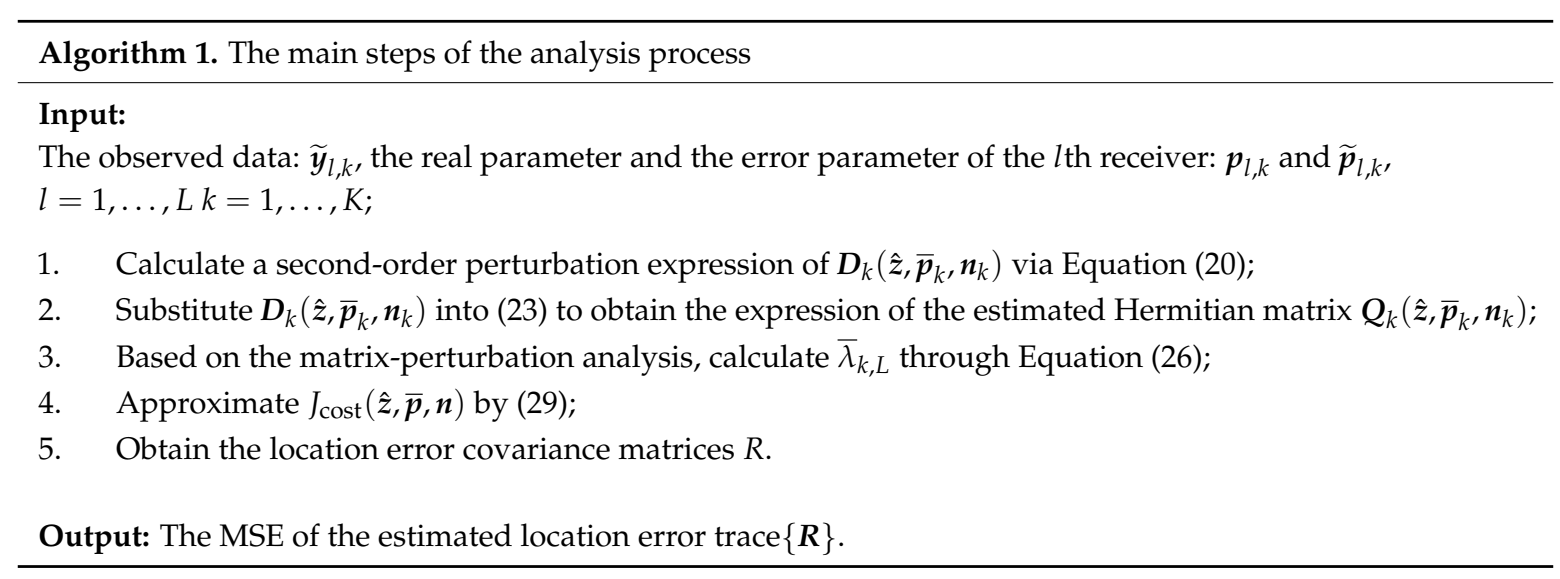

\subsection{CRB under Precise Known Receiver Conditions}

For any unbiased estimator, the CRB provides a lower bound on emitter localization variance. This section presents the derivation of the CRB under the precise known positions and velocities of the receivers. It is not difficult to find that although the MSE in Section 5.3 is given with system errors, the CRB is provided without system errors. Therefore, the comparison between this CRB and the MSE can reveal the performance difference caused by system errors. 
The unknown parameter vector $\eta$ can be defined by

$$
\eta=\left[z^{\mathrm{T}}, \omega^{\mathrm{T}}\right]^{\mathrm{T}}
$$

where $\omega$ denotes all real parameters except the target position. The expression of $\omega$ is written as

$$
\omega=\left[s^{\mathrm{T}}, \boldsymbol{b}^{\mathrm{T}}, \Delta \boldsymbol{f}^{\mathrm{T}}\right]^{\mathrm{T}}
$$

here $s=\left[s_{1}^{(R) \mathrm{T}}, \boldsymbol{s}_{2}^{(R) \mathrm{T}}, \ldots, \boldsymbol{s}_{K}^{(R) \mathrm{T}}, \boldsymbol{s}_{1}^{(I) \mathrm{T}}, \boldsymbol{s}_{2}^{(I) \mathrm{T}}, \ldots, \boldsymbol{s}_{K}^{(I) \mathrm{T}}\right]^{\mathrm{T}}$ with $\boldsymbol{s}_{k}=\left[\boldsymbol{s}_{k}(1), s_{k}(2), \ldots, s_{k}(N)\right]^{\mathrm{T}}$, $\boldsymbol{b}=\left[\boldsymbol{b}_{1}^{(R) \mathrm{T}}, \boldsymbol{b}_{2}^{(R) \mathrm{T}}, \ldots, \boldsymbol{b}_{L}^{(R) \mathrm{T}}, \boldsymbol{b}_{1}^{(I) \mathrm{T}}, \boldsymbol{b}_{2}^{(I) \mathrm{T}}, \ldots, \boldsymbol{b}_{L}^{(I) \mathrm{T}}\right]^{\mathrm{T}}$ with $\boldsymbol{b}_{l}=\left[b_{l, 1}, b_{l, 2}, \ldots, b_{l, K}\right]^{\mathrm{T}}$, and $\Delta \boldsymbol{f}=$ $\left[\Delta f_{1}, \Delta f_{2}, \ldots, \Delta f_{K}\right]^{\mathrm{T}}$.

Let $\boldsymbol{d}_{l, k}(\boldsymbol{\eta})=\boldsymbol{b}_{l, k} \boldsymbol{C}\left(\boldsymbol{z}, \boldsymbol{p}_{l, k}\right) \boldsymbol{B}_{k} \boldsymbol{s}_{k}$. According to [28], the fisher information matrix of unknown parameter vector $\eta$ is shown as

$$
\boldsymbol{J}_{\eta \eta}=\frac{2}{\sigma^{2}} \sum_{k=1}^{K} \sum_{l=1}^{L} \operatorname{Re}\left(\frac{\partial \boldsymbol{d}_{l, k}(\boldsymbol{\eta})}{\partial \boldsymbol{\eta}^{\mathrm{T}}}\right)^{\mathrm{H}}\left(\frac{\partial \boldsymbol{d}_{l, k}(\boldsymbol{\eta})}{\partial \boldsymbol{\eta}^{\mathrm{T}}}\right)
$$

Define

$$
\left\{\begin{array}{c}
\boldsymbol{Y}_{z z}=\sum_{k=1}^{K} \sum_{l=1}^{L} \operatorname{Re}\left\{\left(\frac{d_{l, k}(\eta)}{z^{\mathrm{T}}}\right)^{\mathrm{H}} \frac{d_{l, k}(\eta)}{z^{\mathrm{T}}}\right\} \\
\boldsymbol{Y}_{z \omega}=\sum_{k=1}^{K} \sum_{l=1}^{L} \operatorname{Re}\left\{\left(\frac{d_{l, k}(\eta)}{z^{\mathrm{T}}}\right)^{\mathrm{H}} \frac{d_{l, k}(\eta)}{\omega^{\mathrm{T}}}\right\} \\
\boldsymbol{Y}_{\boldsymbol{\omega} \omega}=\sum_{k=1}^{K} \sum_{l=1}^{L} \operatorname{Re}\left\{\left(\frac{d_{l, k}(\eta)}{\omega^{\mathrm{T}}}\right)^{\mathrm{H}} \frac{d_{l, k}(\eta)}{\omega^{\mathrm{T}}}\right\}
\end{array}\right.
$$

The expression of $J_{\eta \eta}$ can be rewritten as

$$
J_{\eta \eta}=\frac{2}{\sigma_{n}^{2}}\left[\begin{array}{c}
Y_{z z} Y_{z \omega} \\
Y_{z \omega}^{T} Y_{\omega \omega}
\end{array}\right]
$$

Following the matrix inversion formula in Reference [29], the block matrix form of $J_{\eta \eta}$ is formulated as

$$
C R B=\frac{\sigma_{n}^{2}}{2}\left(Y_{z z}-Y_{z w} Y_{w w}^{-1} Y_{z w}^{T}\right)^{-1}
$$

Therefore, substituting the sub-blocks into (56), which are shown in Appendix E, will get the CRB value.

\section{Simulation Results}

This section provides 200 Monte Carlo trials to corroborate the above theoretical analysis based on MATLAB 2015b (MathWorks, Natick, MA, USA), and source data is generated as a Gaussian random signal. Firstly, the localization performance of the proposed method and Weiss's method [10] are performed. Secondly, when system errors exist, the related theoretical values developed in Section 5 are exhibited. Unless otherwise specified, we collect $N=32$ sample points in each interval at a sampling rate of $f_{s}=15 \mathrm{kHz}$, use $L=3$ receivers, perform a total of $K=8$ observations, set the velocity of receiver as $v=300 \mathrm{~m} / \mathrm{s}$ and select the unknown transmitted frequency from [ -100100$] \mathrm{Hz}$ randomly. Additionally, the propagation channel is an additive white Gaussian noise channel, and the channel attenuation is drawn from a normal distribution with mean of 1 and standard deviation of 0.1 , as well as the channel phase is selected from a uniform distribution over $[-\pi, \pi]$. The target locates at [1.5 1.5] $\mathrm{km}$, and the receivers move along the trajectories (three scenarios are included) shown in Figure 1. Note that the simulations in Sections 6.2 and 6.3 are based on the scenario (a) in Figure 1. Finally, 
root mean square error (RMSE) is adopted to evaluate localization accuracy in this paper, which is defined by

$$
R M S E=\sqrt{\frac{1}{200} \sum_{j=1}^{200}\left\|z-\hat{z}^{(j)}\right\|^{2}}
$$

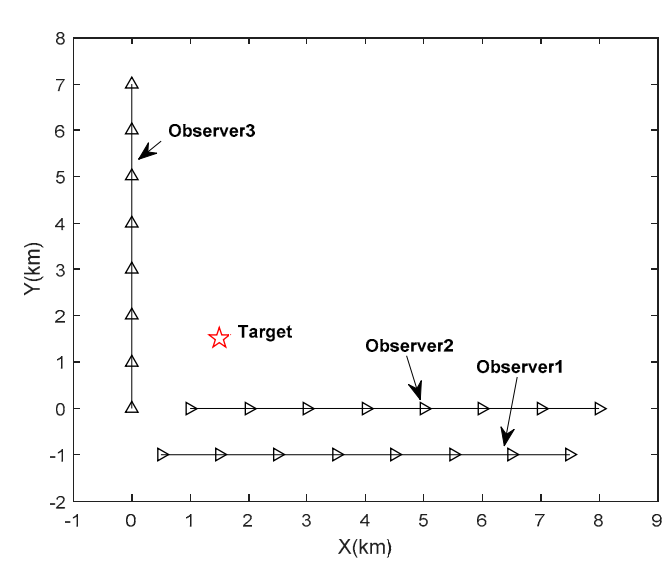

(a)

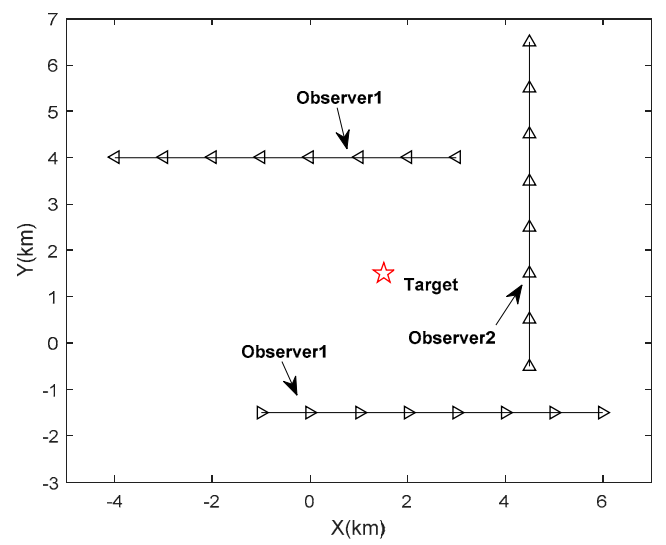

(b)

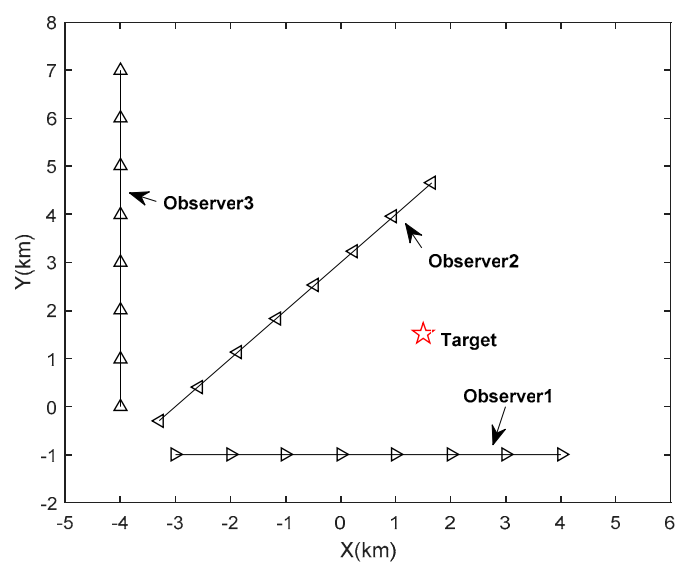

(c)

Figure 1. Position of target and the trajectories of receivers. (a) Scenario a; (b) scenario b; (c) scenario c.

\subsection{Effect of Reveicer Trajectories}

In order to test the test whether our algorithm is sensitive to motion trajectories, we exhibit the localization performance in the different scenarios in Figure 1. Figure 2 indicts that CRB for scenario (a) can generate best localization accuracy, and CRBs for scenario (b) as well as (c) have similar positioning precision. It is easily found that our method has the same trend as with CRB curves. Consequently, the performance of our method is satisfied with theoretical analysis and our method is robust to the receiver trajectories. 


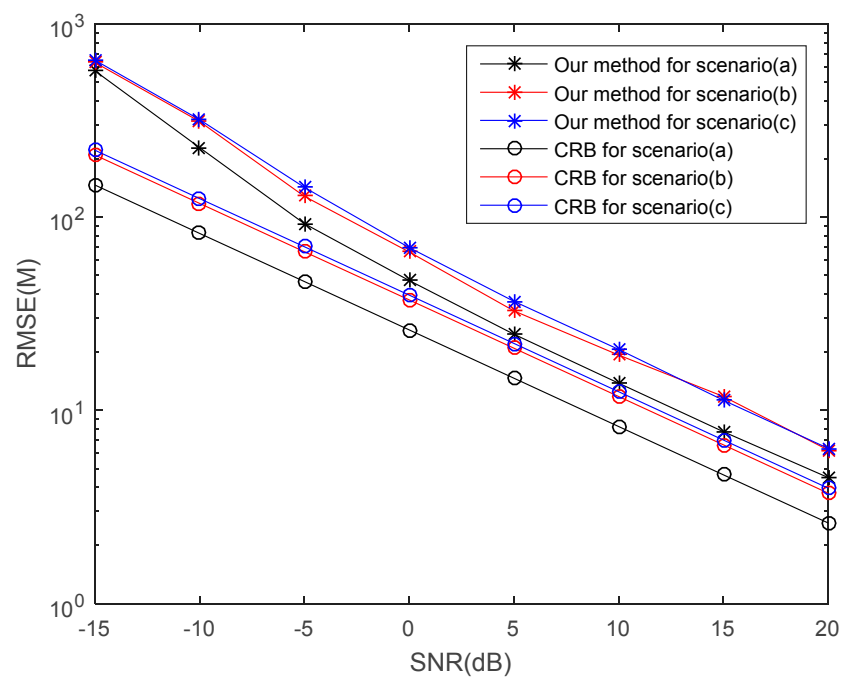

Figure 2. RMSEs versus SNR under different trajectories of receivers.

\subsection{Effect of DOA Information}

To verify the influence of investigating DOA information in signal model on localization performance, we take the following simulations. Firstly, the pseudo spatial spectra of the DPD estimator with different parameter information at $\mathrm{SNR}=-10 \mathrm{~dB}$ are presented in Figure 3. It is easily observed in Figure $3 \mathrm{a}, \mathrm{b}$ that by using additional DOA information, the true peak of the spectrum is more prominent and the pseudo peaks are significantly reduced. Additionally, the 2D plots in Figure $3 c$,d indicate that with the utilization of DOA information, the estimated target position is closer to the true target position.

(a) Our method(3D)

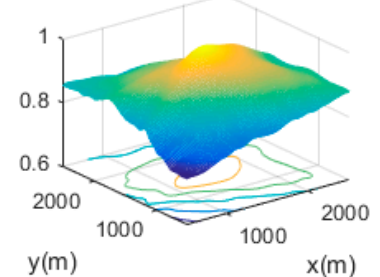

(c) Our method(2D) (b) Weiss's method(3D)

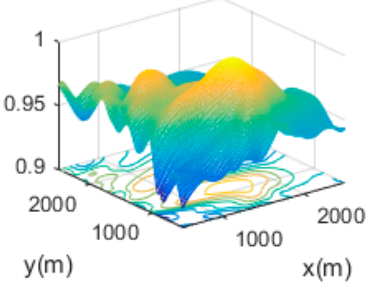

(d) Weiss's method(2D)

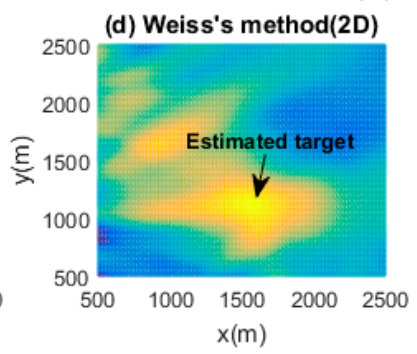

Figure 3. Pseudo spatial spectra of the DPD method using different parameter information. (a) Our method (3D); (b) Welss's method (3D); (c) Our method (2D); (d) Welss's method (2D).

Secondly, the performance comparison between the two methods is available in Figure 4. It is straightforward to see that compared with Weiss's method, our method performs superior at each SNR level. More specifically, our method receives higher localization performance at low SNRs, which shows strong robustness to harsh environments. Additionally, our method is closer to the corresponding CRB. Consequently, DOA information gives a significant improvement on positioning accuracy of this single-step approach. 


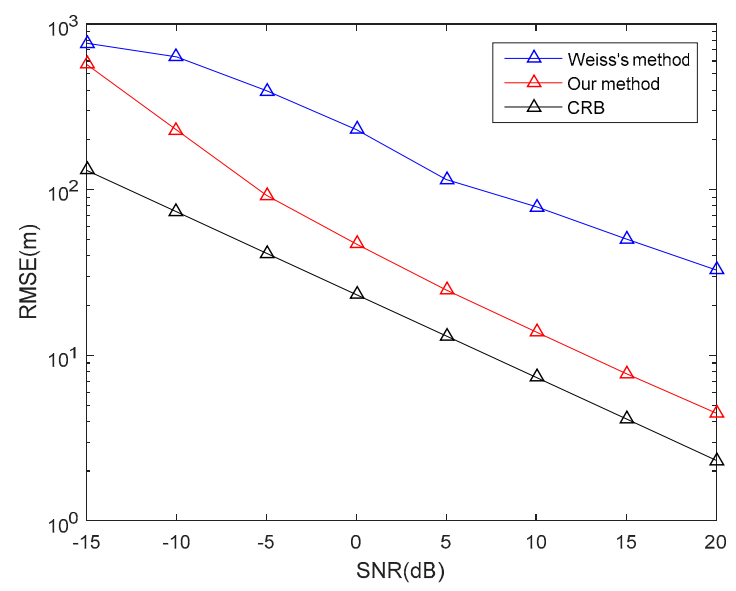

Figure 4. RMSEs versus SNR.

\subsection{Effect of System Errors}

This subsection mainly reveals the performance loss caused by system errors. The disturbances from the receiver position and velocity are assumed to be a Gaussian distribution with zero-mean and variances of $\sigma_{p}^{2}$ as well as $\sigma_{v}^{2}$, respectively. Note that the disturbances from different receivers at different observed interval have the same value in this paper. Additionally, the single-step method is exhibited at two conditions: (1) both system errors and environment noise present; (2) only environment noise attends. Furthermore, the MSE with system errors provided by (50) and the CRB without system errors provided by (56) are also included in the simulations.

Firstly, the localization performance versus SNR are presented and both $\sigma_{p}^{2}$ and $\sigma_{v}^{2}$ are set at 15 . As shown in Figure 5, whether system errors exist or not, there is no difference of the DPD localization performance at SNR ranging from -5 to $0 \mathrm{~dB}$. This phenomenon indicates that positioning accuracy has not received too much influence on system error and is mainly caused by environment noise at low SNRs. However, as SNR increases, the localization performance in presence of system errors deteriorates. It tells us that the localization errors are affected by environment noise and system errors together at high SNRs. Additionally, when SNR reaches $20 \mathrm{~dB}$, the RMSE of our algorithm is almost constant. The reason is that when SNR is relatively large, the localization precision mainly comes from system errors and cannot be reduced by the increase of SNR. Meanwhile, the localization errors of Weiss's method continue to decline, which implies our method can achieve the best performance faster in presence of system errors at the same SNR condition. Furthermore, when two errors exist, the curve of our method approximates the MSE curve, demonstrating the effectiveness of the theoretical analysis in Section 5.

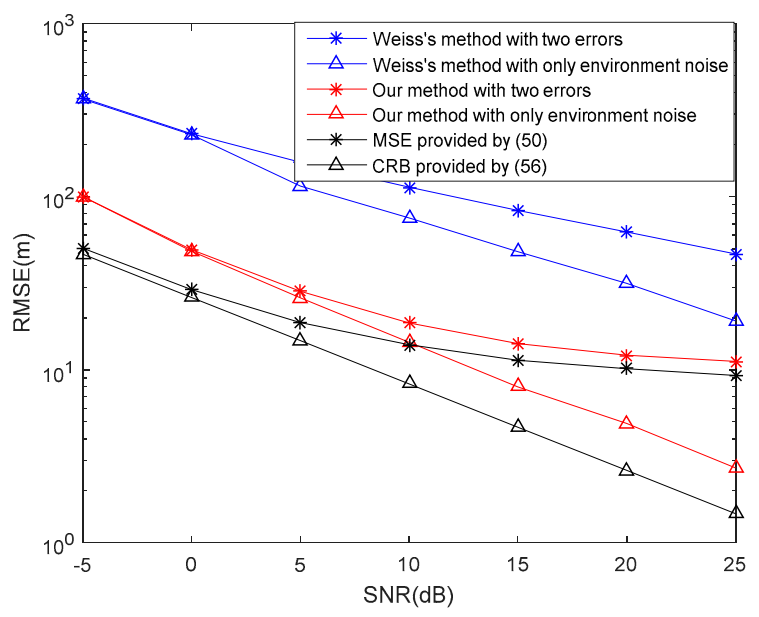

Figure 5. RMSEs versus SNR under different scenarios. 
Then, the localization errors versus the perturbation variance of system errors at $\mathrm{SNR}=10 \mathrm{~dB}$ are plotted in Figure 6. Unsurprisingly, the localization errors of the CRB and our method in absence of system errors have hardly changed. On the other hand, it is evidently seen that the curves of the MSE and our method with two errors are on the rise. The reason is that the DPD estimator could not solve the influence of system errors.

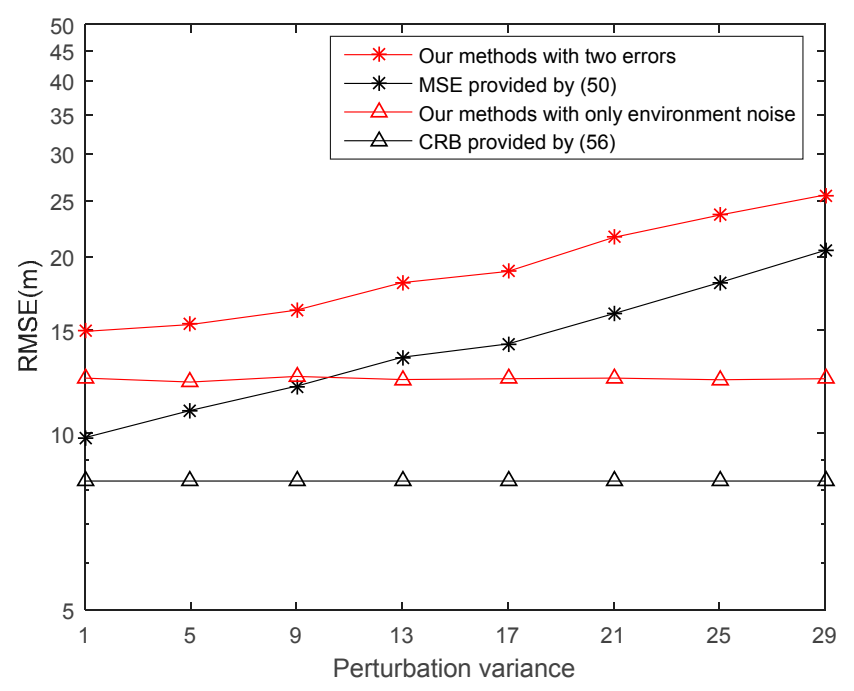

Figure 6. RMSEs versus perturbation variance of system errors.

Finally, in Figure 7, the localization RMSEs versus the number of snapshots is provided, under the scenario that SNR is 10, and both $\sigma_{p}^{2}$ as well as $\sigma_{v}^{2}$ are set at 20. As snapshots increase, the localization performance of the CRB and our method without system errors can improve continuously. Unfortunately, the DPD method remains approximately changeless no matter how much snapshots increase. As we mentioned above, this phenomenon can be explained as system errors being the main contributor to positioning precision under this condition, whose affects cannot be erased by the DPD approach.

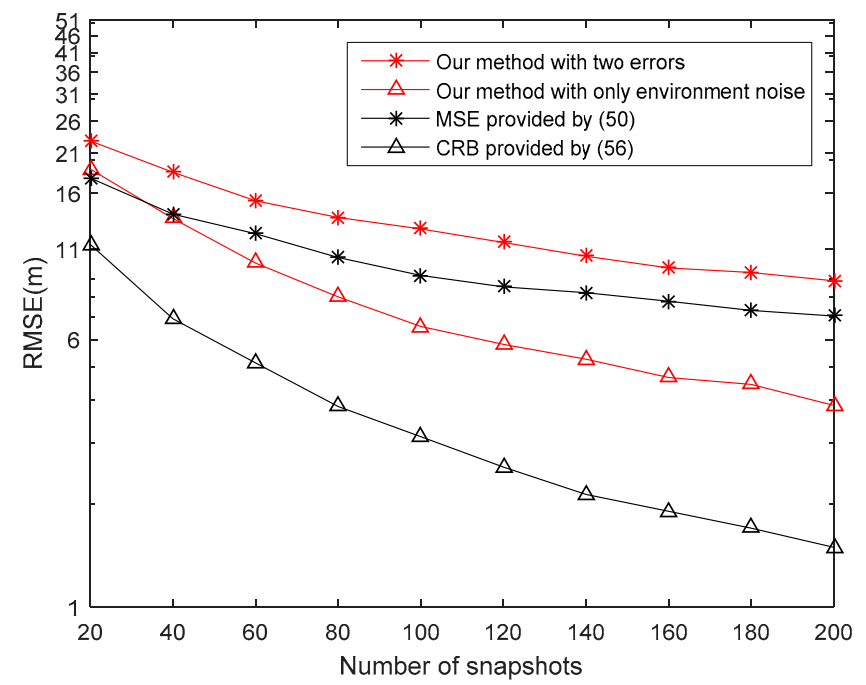

Figure 7. RMSEs versus number of snapshots.

\section{Conclusions}

In this paper, an improved work to the DPD method proposed by Weiss [10] is studied, and the performance analysis of this method with system errors is provided. We start to reconstruct the 
signal model by using Doppler and DOA information, which is more suitable for the moving arrays application. Then, the theoretical analysis is presented based on matrix eigen-perturbation results, which express the perturbations as an additive noise on the Hertmitian matrix. Besides, the MSE formulation of direct localization with system errors is provided. Finally, the CRB formulation for the single-step method is also derived, which indicates the localization performance loss caused by system errors. Several simulations demonstrate the analysis that system errors can deteriorate the localization performance of the DPD estimator especially in high SNRs. Consequently, an improved DPD approach considering system errors should be developed in the future work.

Author Contributions: T.Q. derived the proposed method. T.Q. conceived and designed the experiments. B.B. performed the simulations. D.W. analyzed the results. T.Q. wrote the paper. B.B. reviewed the paper.

Funding: This research was funded by the National Natural Science Foundation of China, grant number 61401513 and The APC was funded by 61401513.

Conflicts of Interest: The authors declare no conflict of interest.

\section{Appendix A. Derivation of The Expressions in (21)}

A second-order Taylor series expansion of $\overline{\boldsymbol{C}}\left(\hat{z}, \overline{\boldsymbol{p}}_{k}\right)$ around $\left(\boldsymbol{z}, \boldsymbol{p}_{k}\right)$ is shown as

$$
\begin{aligned}
\overline{\boldsymbol{C}}\left(\hat{\boldsymbol{z}}, \overline{\boldsymbol{p}}_{k}\right)= & \overline{\boldsymbol{C}}\left(\boldsymbol{z}, \boldsymbol{p}_{k}\right)+\sum_{d=1}^{D}\langle\widetilde{\boldsymbol{z}}\rangle_{d} \dot{\overline{\boldsymbol{C}}}_{d}^{(a)}\left(\boldsymbol{z}, \boldsymbol{p}_{k}\right)+\sum_{d=1}^{2 D L}\left\langle\widetilde{\boldsymbol{p}}_{k}\right\rangle_{d} \dot{\overline{\boldsymbol{C}}}_{d}^{(b)}\left(\boldsymbol{z}, \boldsymbol{p}_{k}\right)+ \\
& \frac{1}{2} \sum_{d_{1}=1}^{D} \sum_{d_{2}=1}^{D}\langle\widetilde{\boldsymbol{z}}\rangle_{d_{1}}\langle\widetilde{\boldsymbol{z}}\rangle_{d_{2}} \ddot{\overline{\boldsymbol{C}}}_{d_{1} d_{2}}^{(a a)}\left(\boldsymbol{z}, \boldsymbol{p}_{k}\right)+\frac{1}{2} \sum_{d_{1}=1}^{2 D L} \sum_{d_{2}=1}^{2 D L}\left\langle\widetilde{\boldsymbol{p}}_{k}\right\rangle_{d_{1}}\left\langle\widetilde{\boldsymbol{p}}_{k}\right\rangle_{d_{2}} \ddot{\overline{\boldsymbol{C}}}_{d_{1} d_{2}}^{(b b)}\left(\boldsymbol{z}, \boldsymbol{p}_{k}\right)+ \\
& \sum_{d_{1}=1}^{D} \sum_{d_{2}=1}^{2 D L}\langle\widetilde{\boldsymbol{z}}\rangle_{d_{1}}\left\langle\widetilde{\boldsymbol{p}}_{k}\right\rangle_{d_{2}} \ddot{\overline{\boldsymbol{C}}}_{d_{1} d_{2}}^{(a b)}\left(\boldsymbol{z}, \boldsymbol{p}_{k}\right)+o\left(\varepsilon^{2}\right)
\end{aligned}
$$

where $\dot{\overline{\boldsymbol{C}}}_{d}^{(a)}\left(\boldsymbol{z}, \boldsymbol{p}_{k}\right), \dot{\overline{\bar{C}}}_{d}^{(b)}\left(\boldsymbol{z}, \boldsymbol{p}_{k}\right), \ddot{\bar{C}}_{d_{1} d_{2}}^{(a a)}\left(\boldsymbol{z}, \boldsymbol{p}_{k}\right), \ddot{\bar{C}}_{d_{1} d_{2}}^{(b b)}\left(\boldsymbol{z}, \boldsymbol{p}_{k}\right)$ and $\ddot{\bar{C}}_{d_{1} d_{2}}^{(a b)}\left(\boldsymbol{z}, \boldsymbol{p}_{k}\right)$ are exhibited in (22).

Substituting (A1) into (13) leads to

$$
\begin{aligned}
& \boldsymbol{D}_{k}\left(\hat{z}, \overline{\boldsymbol{p}}_{k}, \boldsymbol{n}_{k}\right)=\overline{\boldsymbol{C}}\left(\hat{z}, \overline{\boldsymbol{p}}_{k}\right)\left(\boldsymbol{Y}_{k}+\boldsymbol{N}_{k}\right) \approx \overline{\boldsymbol{C}}\left(\boldsymbol{z}, \boldsymbol{p}_{k}\right) \boldsymbol{Y}_{k}+ \\
& \sum_{d=1}^{D}\langle\widetilde{\boldsymbol{z}}\rangle_{d} \dot{\bar{C}}_{d}^{(a)}\left(\boldsymbol{z}, \boldsymbol{p}_{k}\right) \boldsymbol{Y}_{k}+\sum_{d=1}^{2 D L}\left\langle\widetilde{\boldsymbol{p}}_{k}\right\rangle_{d} \dot{\bar{C}}_{d}^{(b)}\left(\boldsymbol{z}, \boldsymbol{p}_{k}\right) \boldsymbol{Y}_{k}+\overline{\boldsymbol{C}}\left(\boldsymbol{z}, \boldsymbol{p}_{k}\right) \boldsymbol{N}_{k}+ \\
& \frac{1}{2} \sum_{d_{1}=1}^{D} \sum_{d_{2}=1}^{D}\langle\widetilde{\boldsymbol{z}}\rangle_{d_{1}}\langle\widetilde{z}\rangle_{d_{2}} \ddot{\bar{C}}_{d_{1} d_{2}}\left(\boldsymbol{z}, \boldsymbol{z}, \boldsymbol{p}_{k}\right) \boldsymbol{Y}_{k}+\frac{1}{2} \sum_{d_{1}=1}^{2 D L} \sum_{d_{2}=1}^{2 D L}\left\langle\widetilde{\boldsymbol{p}}_{k}\right\rangle_{d_{1}}\left\langle\widetilde{\boldsymbol{p}}_{k}\right\rangle_{d_{2}} \ddot{\boldsymbol{\bullet}}_{d_{1} d_{2}}^{(b b)}\left(\boldsymbol{z}, \boldsymbol{p}_{k}\right) \boldsymbol{Y}_{k}+ \\
& \sum_{d_{1}=1}^{D} \sum_{d_{2}=1}^{2 D L}\langle\widetilde{\boldsymbol{z}}\rangle_{d_{1}}\left\langle\widetilde{\boldsymbol{p}}_{k}\right\rangle_{d_{2}} \stackrel{\bullet}{\bar{C}}_{d_{1} d_{2}}^{(a b)}\left(\boldsymbol{z}, \boldsymbol{p}_{k}\right) \boldsymbol{Y}_{k}+\sum_{d=1}^{D}\langle\widetilde{\boldsymbol{z}}\rangle_{d} \dot{\bar{C}}_{d}^{(a)}\left(\mathrm{z}, \boldsymbol{p}_{k}\right) \boldsymbol{N}_{k}+\sum_{d=1}^{2 D L}\left\langle\widetilde{\boldsymbol{p}}_{k}\right\rangle_{d} \dot{\bar{C}}_{d}^{(b)}\left(\boldsymbol{z}, \boldsymbol{p}_{k}\right) \boldsymbol{N}_{k} \\
& =\boldsymbol{D}_{k}^{(0)}+\widetilde{\boldsymbol{D}}_{k}^{(1)}+\widetilde{\boldsymbol{D}}_{k}^{(2)}
\end{aligned}
$$

This ends the derivation.

\section{Appendix B. Derivation of The Expressions in (22)}

Firstly, we start with developing the required derivatives of $\dot{\delta}^{(a)}\left(z, \boldsymbol{o}_{l, k}\right)$ and $\dot{\boldsymbol{\mu}}^{(a)}\left(\boldsymbol{z}, \boldsymbol{p}_{l, k}\right)$ as

$$
\begin{aligned}
& \dot{\delta}^{(a)}\left(\boldsymbol{z}, \boldsymbol{o}_{l, k}\right)=\frac{\partial \delta\left(\boldsymbol{z}, \boldsymbol{o}_{l, k}\right)}{\partial z}=\frac{\left[\begin{array}{cc}
1 & 0
\end{array}\right]^{\mathrm{T}}}{\left\|\boldsymbol{z}-\boldsymbol{o}_{l, k}\right\|_{2}}-\frac{\left(\boldsymbol{z}^{(x)}-\boldsymbol{o}_{l, k}^{(x)}\right)\left(\boldsymbol{z}-\boldsymbol{o}_{l, k}\right)}{\left\|\boldsymbol{z}-\boldsymbol{o}_{l, k}\right\|_{2}^{3}}
\end{aligned}
$$

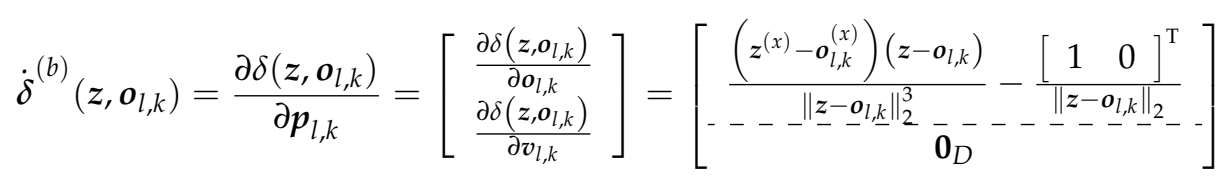




$$
\begin{aligned}
\dot{\delta}^{.(a a)}\left(\boldsymbol{z}, \boldsymbol{o}_{l, k}\right) & =\frac{\partial \delta^{2}\left(z, \boldsymbol{o}_{l, k}\right)}{\partial z \partial z^{\mathrm{T}}} \\
& =3 \frac{\left(\boldsymbol{z}^{(x)}-\boldsymbol{o}_{l, k}^{(x)}\right)\left(z-\boldsymbol{o}_{l, k}\right)\left(z-\boldsymbol{o}_{l, k}\right)^{\mathrm{T}}}{\left\|z-\boldsymbol{o}_{l, k}\right\|_{2}^{5}}-\frac{\left(\boldsymbol{z}^{(x)}-\boldsymbol{o}_{l, k}^{(x)}\right) \boldsymbol{I}_{D}}{\left\|z-\boldsymbol{o}_{l, k}\right\|_{2}^{3}} \\
& -\frac{\left(z-\boldsymbol{o}_{l, k}\right)\left[\begin{array}{ll}
1 & 0
\end{array}\right]}{\left\|z-\boldsymbol{o}_{l, k}\right\|_{2}^{3}}-\frac{\left[\begin{array}{cc}
1 & 0
\end{array}\right]^{\mathrm{T}}\left(z-\boldsymbol{o}_{l, k}\right)^{\mathrm{T}}}{\left\|z-\boldsymbol{o}_{l, k}\right\|_{2}^{3}}
\end{aligned}
$$

$$
\begin{aligned}
\delta^{.(a b)}\left(\boldsymbol{z}, \boldsymbol{o}_{l, k}\right) & =\frac{\partial \delta^{2}\left(\boldsymbol{z}, \boldsymbol{o}_{l, k}\right)}{\partial z \partial \boldsymbol{p}_{l, k}^{\mathrm{T}}} \\
& =\left[\begin{array}{ll}
\frac{\left[\begin{array}{cc}
1 & 0
\end{array}\right]^{\mathrm{T}} \cdot\left(z-\boldsymbol{o}_{l, k}\right)^{\mathrm{T}}}{\left\|z-\boldsymbol{o}_{l, k}\right\|_{2}^{3}}+\frac{\left(z^{(x)}-\boldsymbol{o}_{l, k}^{(x)}\right) \cdot \boldsymbol{I}_{D}}{\left\|z-\boldsymbol{o}_{l, k}\right\|_{2}^{3}}+\frac{\left(z-\boldsymbol{o}_{l, k}\right) \cdot\left[\begin{array}{cc}
1 & 0
\end{array}\right]}{\left\|z-\boldsymbol{o}_{l, k}\right\|_{2}^{3}} & \mathbf{0}_{D} \\
-3 \frac{\left(\boldsymbol{z}^{(x)}-\boldsymbol{o}_{l, k}^{(x)}\right)\left(z-\boldsymbol{o}_{l, k}\right)\left(z-\boldsymbol{o}_{l, k}\right)^{\mathrm{T}}}{\left\|z-\boldsymbol{o}_{l, k}\right\|_{2}^{5}} & \vdots
\end{array}\right]
\end{aligned}
$$

$\ddot{\delta}^{.(b b)}\left(\boldsymbol{z}, \boldsymbol{o}_{l, k}\right)=\frac{\partial \delta^{2}\left(z, \boldsymbol{o}_{l, k}\right)}{\partial \boldsymbol{p}_{l, k} \partial \boldsymbol{p}_{l, k}^{T}}$

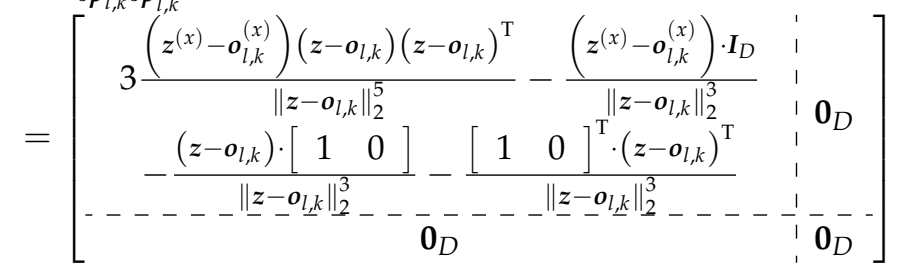

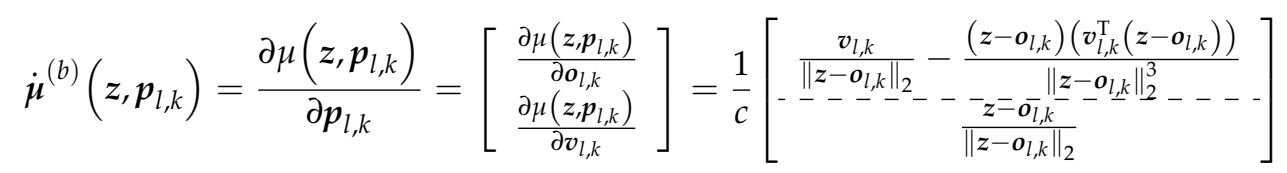

$$
\ddot{\mu}^{.(a a)}\left(z, \boldsymbol{p}_{l, k}\right)=\frac{\partial \mu^{2}\left(z, \boldsymbol{p}_{l, k}\right)}{\partial z \partial z^{\mathrm{T}}}=\frac{1}{c}\left(\begin{array}{l}
3 \frac{\left(\boldsymbol{v}_{l, k}^{\mathrm{T}}\left(z-\boldsymbol{o}_{l, k}\right)\right)}{\left\|z-\boldsymbol{o}_{l, k}\right\|_{2}^{5}}\left(z-\boldsymbol{o}_{l, k}\right)\left(z-\boldsymbol{o}_{l, k}\right)^{\mathrm{T}}-\frac{\boldsymbol{v}_{l, k}\left(z-\boldsymbol{o}_{l, k}\right)^{\mathrm{T}}}{\left\|z-\boldsymbol{o}_{l, k}\right\|_{2}^{3}} \\
-\frac{\boldsymbol{v}_{l, k}^{\mathrm{T}}\left(\boldsymbol{z}-\boldsymbol{o}_{l, k}\right)}{\left\|z-\boldsymbol{o}_{l, k}\right\|_{2}^{3}} \mathbf{I}_{D}-\frac{\left(z-\boldsymbol{o}_{l, k}\right) \boldsymbol{v}_{l, k}^{\mathrm{T}}}{\left\|z-\boldsymbol{o}_{l, k}\right\|_{2}^{3}}
\end{array}\right)
$$

$$
\begin{aligned}
& \ddot{\mu}^{.(a b)}\left(\boldsymbol{z}, \boldsymbol{p}_{l, k}\right)=\frac{\partial \mu^{2}\left(z, \boldsymbol{p}_{l, k}\right)}{\partial z \partial \boldsymbol{p}_{l, k}^{\mathrm{T}}} \\
& =\frac{1}{c}\left[\begin{array}{c:c}
\frac{\boldsymbol{v}_{l, k}\left(z-\boldsymbol{o}_{l, k}\right)^{\mathrm{T}}}{\left\|z-\boldsymbol{o}_{l, k}\right\|_{2}^{3}}+\frac{\boldsymbol{v}_{l, k}^{\mathrm{T}}\left(z-\boldsymbol{o}_{l, k}\right)}{\left\|z-\boldsymbol{o}_{l, k}\right\|_{2}^{3}} \boldsymbol{I}_{D}+\frac{\left(z-\boldsymbol{o}_{l, k}\right) \boldsymbol{v}_{l, k}^{\mathrm{T}}}{\left\|z-\boldsymbol{o}_{l, k}\right\|_{2}^{3}} & \frac{1}{\left\|z-\boldsymbol{o}_{l, k}\right\|_{2}} \boldsymbol{I}_{D}- \\
-3 \frac{\left(\boldsymbol{v}_{l, k}^{\mathrm{T}}\left(\boldsymbol{z}-\boldsymbol{o}_{l, k}\right)\right)}{\left\|\boldsymbol{z}-\boldsymbol{o}_{l, k}\right\|_{2}^{5}}\left(\boldsymbol{z}-\boldsymbol{o}_{l, k}\right)\left(\boldsymbol{z}-\boldsymbol{o}_{l, k}\right)^{\mathrm{T}} & \frac{\left(z-\boldsymbol{o}_{l, k}\right)\left(z-\boldsymbol{o}_{l, k}\right)^{\mathrm{T}}}{\left\|\boldsymbol{z}-\boldsymbol{o}_{l, k}\right\|_{2}^{3}}
\end{array}\right]
\end{aligned}
$$

$$
=\frac{1}{c}\left[\begin{array}{c:c}
\boldsymbol{\mu}^{(b b)}\left(\boldsymbol{z}, \boldsymbol{p}_{l, k}\right)=\frac{\partial \mu^{2}\left(z, \boldsymbol{p}_{l, k}\right)}{\partial \boldsymbol{p}_{l, k} \partial \boldsymbol{p}_{l, k}^{\mathrm{T}}} & \\
-\frac{\boldsymbol{v}_{l, k}^{\mathrm{T}}\left(z-\boldsymbol{o}_{l, k}\right)}{\left\|z-\boldsymbol{o}_{l, k}\right\|_{2}^{3}} \boldsymbol{I}_{D}-\frac{\left(z-\boldsymbol{o}_{l, k}\right) \boldsymbol{v}_{l, k}^{\mathrm{T}}}{\left\|z-\boldsymbol{o}_{l, k}\right\|_{2}^{3}} & \frac{\left(z-\boldsymbol{o}_{l, k}\right)\left(z-\boldsymbol{o}_{l, k}\right)^{\mathrm{T}}}{\left\|z-\boldsymbol{o}_{l, k}\right\|_{2}^{3}} \\
\hdashline \frac{\left(z-\boldsymbol{o}_{l, k}\right)\left(z-\boldsymbol{o}_{l, k}\right)^{\mathrm{T}}}{\left\|z-\boldsymbol{o}_{l, k}\right\|_{2}^{3}} & -\frac{1}{\left\|z-\boldsymbol{o}_{l, k}\right\|_{2}} \boldsymbol{I}_{D} \\
\hdashline-\boldsymbol{o}_{l, k} & 0_{D}^{\mathrm{T}}
\end{array}\right]
$$

Following the expression of $\overline{\boldsymbol{C}}\left(\boldsymbol{z}, \boldsymbol{p}_{k}\right)$ in (14), it gives 
Appendix B.1. Expression of $\dot{\overline{\boldsymbol{C}}}_{d}^{(a)}\left(\boldsymbol{z}, \boldsymbol{p}_{k}\right)$

$$
\dot{\overline{\boldsymbol{C}}}_{d}^{(a)}\left(\boldsymbol{z}, \boldsymbol{p}_{k}\right)=\frac{\partial \overline{\boldsymbol{C}}\left(\boldsymbol{z}, \boldsymbol{p}_{k}\right)}{\partial\langle\boldsymbol{z}\rangle_{d}}=\left[\frac{\partial \boldsymbol{C}^{\mathrm{H}}\left(\boldsymbol{z}, \boldsymbol{p}_{1, k}\right)}{\partial\langle\boldsymbol{z}\rangle_{d}}, \frac{\partial \boldsymbol{C}^{\mathrm{H}}\left(\boldsymbol{z}, \boldsymbol{p}_{2, k}\right)}{\partial\langle\boldsymbol{z}\rangle_{d}}, \ldots, \frac{\partial \boldsymbol{C}^{\mathrm{H}}\left(\boldsymbol{z}, \boldsymbol{p}_{L, k}\right)}{\partial\langle\boldsymbol{z}\rangle_{d}}\right]
$$

where

$$
\frac{\partial C^{\mathrm{H}}\left(\boldsymbol{z}, \boldsymbol{p}_{l, k}\right)}{\partial\langle\boldsymbol{z}\rangle_{d}}=\left[\frac{\partial \boldsymbol{a}\left(\boldsymbol{z}, \boldsymbol{o}_{l, k}\right)}{\partial\langle\boldsymbol{z}\rangle_{d}} \otimes \boldsymbol{A}\left(\boldsymbol{z}, \boldsymbol{p}_{l, k}\right)+\boldsymbol{a}\left(\boldsymbol{z}, \boldsymbol{o}_{l, k}\right) \otimes \frac{\partial A\left(\boldsymbol{z}, \boldsymbol{p}_{l, k}\right)}{\partial\langle\boldsymbol{z}\rangle_{d}}\right]^{\mathrm{H}}
$$

with

$$
\begin{gathered}
\frac{\partial \boldsymbol{a}\left(\boldsymbol{z}, \boldsymbol{o}_{l, k}\right)}{\partial\langle\boldsymbol{z}\rangle_{d}}=\dot{\boldsymbol{\psi}}_{1, d}^{(a)}\left(\boldsymbol{z}, \boldsymbol{o}_{l, k}\right) \cdot \boldsymbol{a}\left(\boldsymbol{z}, \boldsymbol{o}_{l, k}\right) \\
\dot{\boldsymbol{\psi}}_{1, d}^{(a)}\left(\boldsymbol{z}, \boldsymbol{o}_{l, k}\right)=\left\langle\dot{\boldsymbol{\delta}}^{(a)}\left(\boldsymbol{z}, \boldsymbol{o}_{l, k}\right)\right\rangle_{d} \cdot \operatorname{diag}\left\{j 2 \pi \frac{d}{\lambda} \widetilde{\boldsymbol{M}}\right\}
\end{gathered}
$$

and

$$
\begin{gathered}
\frac{\partial A\left(z, p_{l, k}\right)}{\partial\langle z\rangle_{d}}=A\left(z, p_{l, k}\right) \cdot \dot{\psi}_{2, d}^{(a)}\left(z, p_{l, k}\right) \\
\dot{\psi}_{2, d}^{(a)}\left(z, p_{l, k}\right)=\left\langle\dot{\mu}^{(a)}\left(z, p_{l, k}\right)\right\rangle_{d} \cdot \operatorname{diag}\left\{j 2 \pi f_{c} \widetilde{\boldsymbol{N}} T_{s}\right\}
\end{gathered}
$$

Appendix B.2. Expression of $\dot{\overline{\boldsymbol{C}}}_{d}^{(b)}\left(\boldsymbol{z}, \boldsymbol{p}_{k}\right)$

$$
\dot{\overline{\bar{C}}}_{d}^{(b)}\left(\boldsymbol{z}, \boldsymbol{p}_{k}\right)=\frac{\partial \overline{\boldsymbol{C}}\left(\boldsymbol{z}, \boldsymbol{p}_{k}\right)}{\partial\left\langle\boldsymbol{p}_{k}\right\rangle_{d}}=\left[\frac{\partial \boldsymbol{C}^{\mathrm{H}}\left(\boldsymbol{z}, \boldsymbol{p}_{1, k}\right)}{\partial\left\langle\boldsymbol{p}_{k}\right\rangle_{d}}, \frac{\partial \boldsymbol{C}^{\mathrm{H}}\left(\boldsymbol{z}, \boldsymbol{p}_{2, k}\right)}{\partial\left\langle\boldsymbol{p}_{k}\right\rangle_{d}}, \ldots, \frac{\partial \boldsymbol{C}^{\mathrm{H}}\left(\boldsymbol{z}, \boldsymbol{p}_{L, k}\right)}{\partial\left\langle\boldsymbol{p}_{k}\right\rangle_{d}}\right]
$$

where

$$
\frac{\partial \boldsymbol{C}^{\mathrm{H}}\left(\boldsymbol{z}, \boldsymbol{p}_{l, k}\right)}{\partial\left\langle\boldsymbol{p}_{k}\right\rangle_{d}}=\left[\frac{\partial \boldsymbol{a}\left(\boldsymbol{z}, \boldsymbol{o}_{l, k}\right)}{\partial\left\langle\boldsymbol{p}_{k}\right\rangle_{d}} \otimes \boldsymbol{A}\left(\boldsymbol{z}, \boldsymbol{p}_{l, k}\right)+\boldsymbol{a}\left(\boldsymbol{z}, \boldsymbol{o}_{l, k}\right) \otimes \frac{\partial \boldsymbol{A}\left(\boldsymbol{z}, \boldsymbol{p}_{l, k}\right)}{\partial\left\langle\boldsymbol{p}_{k}\right\rangle_{d}}\right]^{\mathrm{H}}
$$

with

$$
\begin{gathered}
\frac{\partial \boldsymbol{a}\left(\boldsymbol{z}, \boldsymbol{o}_{l, k}\right)}{\partial\left\langle\boldsymbol{p}_{k}\right\rangle_{d}}=\dot{\boldsymbol{\psi}}_{1, d}^{(b)}\left(\boldsymbol{z}, \boldsymbol{o}_{l, k}\right) \cdot \boldsymbol{a}\left(\boldsymbol{z}, \boldsymbol{o}_{l, k}\right) \\
\dot{\boldsymbol{\psi}}_{1, d}^{(b)}\left(\boldsymbol{z}, \boldsymbol{o}_{l, k}\right)=\left\langle\dot{\boldsymbol{\delta}}^{(b)}\left(\boldsymbol{z}, \boldsymbol{o}_{l, k}\right)\right\rangle_{d} \cdot \operatorname{diag}\left\{j 2 \pi \frac{d}{\lambda} \widetilde{\boldsymbol{M}}\right\}
\end{gathered}
$$

and

$$
\begin{gathered}
\frac{\partial A\left(z, p_{l, k}\right)}{\partial\left\langle p_{k}\right\rangle_{d}}=A\left(z, p_{l, k}\right) \cdot \dot{\psi}_{2, d}^{(b)}\left(z, p_{l, k}\right) \\
\dot{\psi}_{2, d}^{(b)}\left(z, p_{l, k}\right)=\left\langle\dot{\mu}^{(b)}\left(z, p_{l, k}\right)\right\rangle_{d} \cdot \operatorname{diag}\left\{j 2 \pi f_{c} \widetilde{\boldsymbol{N}} T_{s}\right\}
\end{gathered}
$$


therefore $\dot{\bar{C}}_{d}^{(b)}\left(\boldsymbol{z}, \boldsymbol{p}_{k}\right)$ can be written as

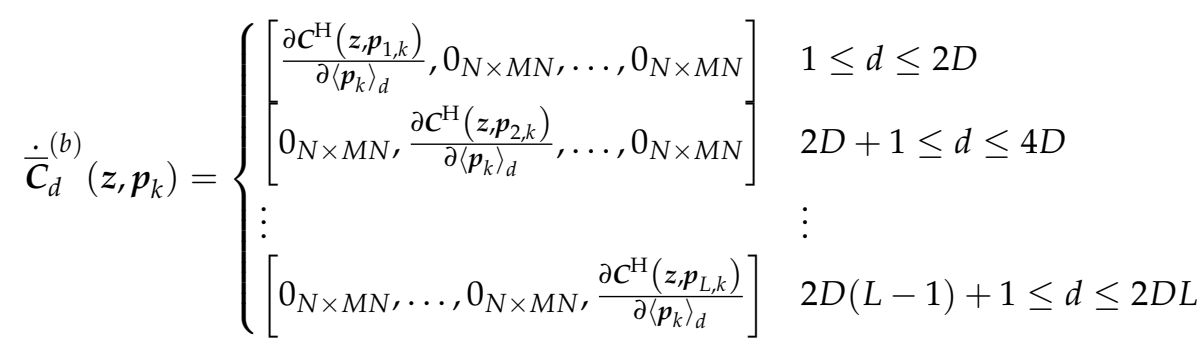

Appendix B.3. Expression of $\stackrel{\cdot(a a)}{\overline{\boldsymbol{C}}_{d_{1} d_{2}}\left(\boldsymbol{z}, \boldsymbol{p}_{k}\right)}$

$$
\overline{\boldsymbol{C}}_{d_{1} d_{2}}\left(\boldsymbol{z}, \boldsymbol{p}_{k}\right)=\frac{\partial^{2} \overline{\boldsymbol{C}}\left(\boldsymbol{z}, \boldsymbol{p}_{k}\right)}{\partial\langle\boldsymbol{z}\rangle_{d_{1}} \partial\langle\boldsymbol{z}\rangle_{d_{2}}}=\left[\frac{\partial^{2} \boldsymbol{C}^{\mathrm{H}}\left(\boldsymbol{z}, \boldsymbol{p}_{1, k}\right)}{\partial\langle\boldsymbol{z}\rangle_{d_{1}} \partial\langle\boldsymbol{z}\rangle_{d_{2}}}, \frac{\partial^{2} \boldsymbol{C}^{\mathrm{H}}\left(\boldsymbol{z}, \boldsymbol{p}_{2, k}\right)}{\partial\langle\boldsymbol{z}\rangle_{d_{1}} \partial\langle\boldsymbol{z}\rangle_{d_{2}}}, \ldots, \frac{\partial^{2} \boldsymbol{C}^{\mathrm{H}}\left(\boldsymbol{z}, \boldsymbol{p}_{L, k}\right)}{\partial\langle\boldsymbol{z}\rangle_{d_{1}} \partial\langle\boldsymbol{z}\rangle_{d_{2}}}\right]
$$

where

$$
\frac{\partial^{2} \boldsymbol{C}^{\mathrm{H}}\left(\boldsymbol{z}, \boldsymbol{p}_{l, k}\right)}{\partial\langle\boldsymbol{z}\rangle_{d_{1}} \partial\langle\boldsymbol{z}\rangle_{d_{2}}}=\left[\begin{array}{c}
\frac{\partial^{2} \boldsymbol{a}\left(\boldsymbol{z}, \boldsymbol{o}_{l, k}\right)}{\partial\langle\boldsymbol{z}\rangle_{d_{1}} \partial\langle\boldsymbol{z}\rangle_{d_{2}}} \otimes A\left(\boldsymbol{z}, \boldsymbol{p}_{l, k}\right)+\frac{\partial \boldsymbol{a}\left(\boldsymbol{z}, \boldsymbol{o}_{l, k}\right)}{\partial\langle\boldsymbol{z}\rangle_{d_{1}}} \otimes \frac{\partial A\left(z, \boldsymbol{p}_{l, k}\right)}{\partial\langle\boldsymbol{z}\rangle_{d_{2}}} \\
+\frac{\partial \boldsymbol{a}\left(\boldsymbol{z}, \boldsymbol{o}_{l, k}\right)}{\partial\langle\boldsymbol{z}\rangle_{d_{2}}} \otimes \frac{\partial A\left(z, p_{l, k}\right)}{\partial\langle\boldsymbol{z}\rangle_{d_{1}}}+\boldsymbol{a}\left(\boldsymbol{z}, \boldsymbol{o}_{l, k}\right) \otimes \frac{\partial^{2} A\left(z, p_{l, k}\right)}{\partial\langle\boldsymbol{z}\rangle_{d_{1}} \partial\langle\boldsymbol{z}\rangle_{d_{2}}}
\end{array}\right]^{\mathrm{H}}
$$

with

$$
\begin{aligned}
\frac{\partial^{2} a\left(z, \boldsymbol{o}_{l, k}\right)}{\partial\langle\boldsymbol{z}\rangle_{d_{1}} \partial\langle\boldsymbol{z}\rangle_{d_{2}}} & =\dot{\boldsymbol{\psi}}_{1, d_{1}}^{(a)}\left(\boldsymbol{z}, \boldsymbol{o}_{l, k}\right) \cdot \frac{\partial a\left(z, \boldsymbol{o}_{l, k}\right)}{\partial\langle\boldsymbol{z}\rangle_{d_{2}}}+\boldsymbol{a}\left(\boldsymbol{z}, \boldsymbol{o}_{l, k}\right) \cdot \frac{\partial \dot{\boldsymbol{\psi}}_{1, d_{1}}^{(a)}\left(z, \boldsymbol{o}_{l, k}\right)}{\partial\langle\boldsymbol{z}\rangle_{d_{2}}} \\
& =\dot{\boldsymbol{\psi}}_{1, d_{1}}^{(a)}\left(\boldsymbol{z}, \boldsymbol{o}_{l, k}\right) \dot{\boldsymbol{\psi}}_{1, d_{2}}^{(a)}\left(\boldsymbol{z}, \boldsymbol{o}_{l, k}\right) \boldsymbol{a}\left(\boldsymbol{z}, \boldsymbol{o}_{l, k}\right)+\boldsymbol{a}\left(\boldsymbol{z}, \boldsymbol{o}_{l, k}\right) \boldsymbol{\psi}_{1, d_{1} d_{2}}\left(\boldsymbol{z}, \boldsymbol{o}_{l, k}\right) \\
& . \cdot(a a) \\
& \dot{\psi}_{1, d_{1} d_{2}}\left(\boldsymbol{z}, \boldsymbol{o}_{l, k}\right)=\left\langle\dot{\boldsymbol{\delta}}^{\cdot(a a)}\left(\boldsymbol{z}, \boldsymbol{o}_{l, k}\right)\right\rangle_{d_{1} d_{2}} \cdot \operatorname{diag}\left\{j 2 \pi \frac{d}{\lambda} \tilde{\boldsymbol{M}}\right\}
\end{aligned}
$$

and

$$
\begin{aligned}
\frac{\partial^{2} A\left(z, p_{l, k}\right)}{\partial\langle z\rangle_{d_{1}} \partial\langle z\rangle_{d_{2}}}= & A\left(z, p_{l, k}\right) \cdot \frac{\partial \dot{\psi}_{2, d_{1}}^{(a)}\left(z, p_{l, k}\right)}{\partial\langle z\rangle_{d_{2}}}+\dot{\psi}_{2, d_{1}}^{(a)}\left(z, p_{l, k}\right) \cdot \frac{\partial A\left(z, p_{l, k}\right)}{\partial\langle z\rangle_{d_{2}}} \\
= & A\left(z, p_{l, k}\right) \dot{\psi}_{2, d_{1} d_{2}}\left(z, \boldsymbol{p}_{l, k}\right)+\dot{\psi}_{2, d_{1}}^{(a)}\left(z, \boldsymbol{p}_{l, k}\right) \boldsymbol{A}\left(\boldsymbol{z}, \boldsymbol{p}_{l, k}\right) \dot{\psi}_{2, d_{2}}^{(a)}\left(\boldsymbol{z}, \boldsymbol{p}_{l, k}\right) \\
& . .(a a) \\
& \boldsymbol{\psi}_{2, d_{1} d_{2}}\left(\boldsymbol{z}, \boldsymbol{p}_{l, k}\right)=\left\langle\boldsymbol{\mu}^{(a a)}\left(\boldsymbol{z}, \boldsymbol{p}_{l, k}\right)\right\rangle_{d_{1} d_{2}} \cdot \operatorname{diag}\left\{j 2 \pi f_{c} \tilde{\boldsymbol{N}} T_{s}\right\}
\end{aligned}
$$

.. (ab)

Appendix B.4. Expression of $\overline{\boldsymbol{C}}_{d_{1} d_{2}}\left(\boldsymbol{z}, \boldsymbol{p}_{k}\right)$

$$
\begin{aligned}
\overline{\boldsymbol{C}}_{d_{1} d_{2}}\left(\boldsymbol{z}, \boldsymbol{p}_{k}\right) & =\frac{\partial^{2} \overline{\boldsymbol{C}}\left(\boldsymbol{z}, \boldsymbol{p}_{k}\right)}{\partial\langle\boldsymbol{z}\rangle_{d_{1}} \partial\left\langle\boldsymbol{p}_{k}\right\rangle_{d_{2}}} \\
& =\left[\frac{\partial^{2} C^{\mathrm{H}}\left(\boldsymbol{z}, \boldsymbol{p}_{1, k}\right)}{\partial\langle\boldsymbol{z}\rangle_{d_{1}} \partial\left\langle\boldsymbol{p}_{k}\right\rangle_{d_{2}}}, \frac{\partial^{2} C^{\mathrm{H}}\left(\boldsymbol{z}, \boldsymbol{p}_{2, k}\right)}{\partial\langle\boldsymbol{z}\rangle_{d_{1}} \partial\left\langle\boldsymbol{p}_{k}\right\rangle_{d_{2}}}, \ldots, \frac{\partial^{2} C^{\mathrm{H}}\left(\boldsymbol{z}, \boldsymbol{p}_{L, k}\right)}{\partial\langle\boldsymbol{z}\rangle_{d_{1}} \partial\left\langle\boldsymbol{p}_{k}\right\rangle_{d_{2}}}\right]
\end{aligned}
$$

where

$$
\frac{\partial^{2} \boldsymbol{C}^{\mathrm{H}}\left(\boldsymbol{z}, \boldsymbol{p}_{l, k}\right)}{\partial\langle\boldsymbol{z}\rangle_{d_{1}} \partial\left\langle\boldsymbol{p}_{k}\right\rangle_{d_{2}}}=\left[\begin{array}{c}
\frac{\partial^{2} \boldsymbol{a}\left(\boldsymbol{z}, \boldsymbol{o}_{l, k}\right)}{\partial\langle\boldsymbol{z}\rangle_{d_{1}} \partial\left\langle\boldsymbol{p}_{k}\right\rangle_{d_{2}}} \otimes A\left(\boldsymbol{z}, \boldsymbol{p}_{l, k}\right)+\frac{\partial \boldsymbol{a}\left(\boldsymbol{z}, \boldsymbol{o}_{l, k}\right)}{\partial\langle\boldsymbol{z}\rangle_{d_{1}}} \otimes \frac{\partial A\left(z, \boldsymbol{p}_{l, k}\right)}{\partial\left\langle\boldsymbol{p}_{k}\right\rangle_{d_{2}}} \\
+\frac{\partial \boldsymbol{a}\left(\boldsymbol{z}, \boldsymbol{o}_{l, k}\right)}{\partial\left\langle\boldsymbol{p}_{k}\right\rangle_{d_{2}}} \otimes \frac{\partial A\left(z, \boldsymbol{p}_{l, k}\right)}{\partial\langle\boldsymbol{z}\rangle_{d_{1}}}+\boldsymbol{a}\left(\boldsymbol{z}, \boldsymbol{o}_{l, k}\right) \otimes \frac{\partial^{2} A\left(z, \boldsymbol{p}_{l, k}\right)}{\partial\langle\boldsymbol{z}\rangle_{d_{1}} \partial\left\langle\boldsymbol{p}_{k}\right\rangle_{d_{2}}}
\end{array}\right]^{\mathrm{H}}
$$


with

$$
\begin{aligned}
& \frac{\partial^{2} a\left(z, o_{l, k}\right)}{\partial\langle z\rangle_{d_{1}} \partial\left\langle p_{k}\right\rangle_{d_{2}}}=\dot{\boldsymbol{\psi}}_{1, d_{1}}^{(a)}\left(z, \boldsymbol{o}_{l, k}\right) \cdot \frac{\partial a\left(z, \boldsymbol{o}_{l, k}\right)}{\partial\left\langle p_{k}\right\rangle_{d_{2}}}+\boldsymbol{a}\left(\boldsymbol{z}, \boldsymbol{o}_{l, k}\right) \cdot \frac{\partial \dot{\psi}_{1, d_{1}}^{(a)}\left(z, \boldsymbol{o}_{l, k}\right)}{\partial\left\langle p_{k}\right\rangle_{d_{2}}} \\
& =\dot{\boldsymbol{\psi}}_{1, d_{1}}^{(a)}\left(\boldsymbol{z}, \boldsymbol{o}_{l, k}\right) \dot{\boldsymbol{\psi}}_{1, d_{2}}^{(b)}\left(\boldsymbol{z}, \boldsymbol{o}_{l, k}\right) \boldsymbol{a}\left(\boldsymbol{z}, \boldsymbol{o}_{l, k}\right)+\boldsymbol{a}\left(\boldsymbol{z}, \boldsymbol{o}_{l, k}\right) \ddot{\boldsymbol{\psi}}_{1, d_{1} d_{2}}\left(\boldsymbol{z}, \boldsymbol{o}_{l, k}\right) \\
& \dot{\psi}_{1, d_{1} d_{2}}\left(\boldsymbol{z}, \boldsymbol{o}_{l, k}\right)=\left\langle\dot{. .(a b)}^{\boldsymbol{\delta}}\left(\boldsymbol{z}, \boldsymbol{o}_{l, k}\right)\right\rangle_{d_{1} d_{2}} \cdot \operatorname{diag}\left\{j 2 \pi \frac{d}{\lambda} \widetilde{\boldsymbol{M}}\right\}
\end{aligned}
$$

and

$$
\begin{aligned}
& \frac{\partial^{2} A\left(z, p_{l, k}\right)}{\partial\langle z\rangle_{d_{1}} \partial\left\langle p_{k}\right\rangle_{d_{2}}}=A\left(z, p_{l, k}\right) \cdot \dot{\psi}_{2, d_{1} d_{2}}^{-(a b)}\left(z, p_{l, k}\right)+\dot{\psi}_{2, d_{1}}^{(a)}\left(z, p_{l, k}\right) \cdot A\left(z, p_{l, k}\right) \cdot \dot{\psi}_{2, d_{2}}^{(b)}\left(z, p_{l, k}\right) \\
& \stackrel{.(a b)}{\boldsymbol{\psi}_{2, d_{1} d_{2}}}\left(\boldsymbol{z}, \boldsymbol{o}_{l, k}\right)=\left\langle\ddot{\mu}^{\cdot(a b)}\left(\boldsymbol{z}, \boldsymbol{p}_{l, k}\right)\right\rangle_{d_{1} d_{2}} \cdot \operatorname{diag}\left\{j 2 \pi f_{c} \widetilde{\boldsymbol{N}} T_{S}\right\}
\end{aligned}
$$

.. (ab)

Therefore, $\overline{\boldsymbol{C}}_{d_{1} d_{2}}\left(\boldsymbol{z}, \boldsymbol{p}_{k}\right)$ can be written as

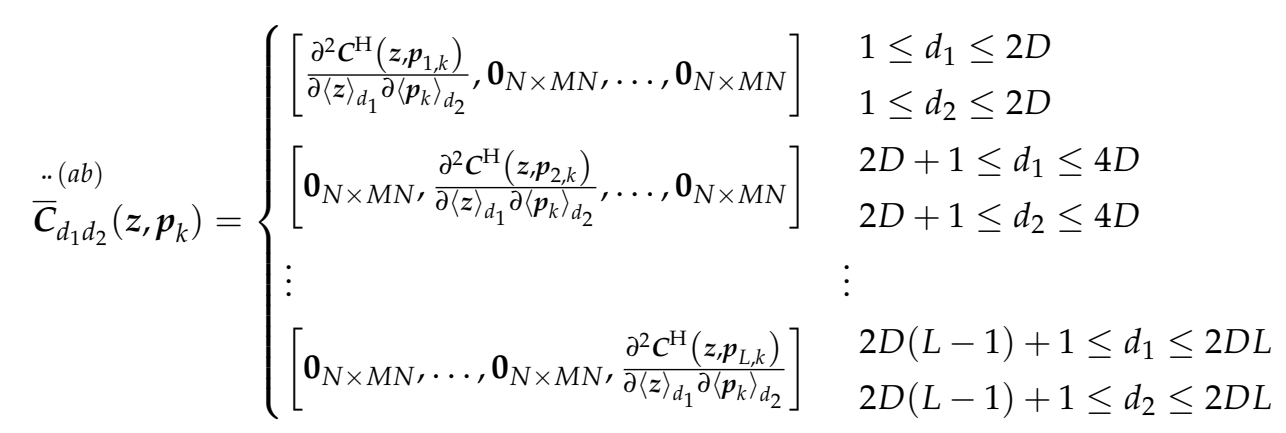

..$(b b)$

Appendix B.5. Expression of $\overline{\boldsymbol{C}}_{d_{1} d_{2}}\left(\boldsymbol{z}, \boldsymbol{p}_{k}\right)$

$$
\begin{aligned}
& \bar{C}_{d_{1} d_{2}}\left(\boldsymbol{z}, \boldsymbol{p}_{k}\right)=\frac{\partial^{2} \bar{C}\left(z, p_{k}\right)}{\partial\left\langle p_{k}\right\rangle_{d_{1}} \partial\left\langle p_{k}\right\rangle_{d_{2}}} \\
& =\left[\frac{\partial^{2} C^{\mathrm{H}}\left(z, p_{1, k}\right)}{\partial\left\langle p_{k}\right\rangle_{d_{1}} \partial\left\langle p_{k}\right\rangle_{d_{2}}}, \frac{\partial^{2} C^{\mathrm{H}}\left(z, p_{2, k}\right)}{\partial\left\langle p_{k}\right\rangle_{d_{1}} \partial\left\langle p_{k}\right\rangle_{d_{2}}}, \ldots, \frac{\partial^{2} C^{\mathrm{H}}\left(z, p_{L, k}\right)}{\partial\left\langle p_{k}\right\rangle_{d_{1}} \partial\left\langle p_{k}\right\rangle_{d_{2}}}\right]
\end{aligned}
$$

where

$$
\frac{\partial^{2} C^{\mathrm{H}}\left(\boldsymbol{z}, \boldsymbol{p}_{l, k}\right)}{\partial\left\langle\boldsymbol{p}_{k}\right\rangle_{d_{1}} \partial\left\langle\boldsymbol{p}_{k}\right\rangle_{d_{2}}}=\left[\begin{array}{c}
\frac{\partial^{2} a\left(z, \boldsymbol{o}_{l, k}\right)}{\partial\left\langle\boldsymbol{p}_{k}\right\rangle_{d_{1}} \partial\left\langle p_{k}\right\rangle_{d_{2}}} \otimes A\left(z, \boldsymbol{p}_{l, k}\right)+\frac{\partial a\left(z, \boldsymbol{o}_{l, k}\right)}{\partial\left\langle p_{k}\right\rangle_{d_{1}}} \otimes \frac{\partial A\left(z, p_{l, k}\right)}{\partial\left\langle p_{k}\right\rangle_{d_{2}}} \\
+\frac{\partial a\left(z, \boldsymbol{o}_{l, k}\right)}{\partial\left\langle\boldsymbol{p}_{k}\right\rangle_{d_{2}}} \otimes \frac{\partial A\left(z, p_{l, k}\right)}{\partial\left\langle p_{k}\right\rangle_{d_{1}}}+\boldsymbol{a}\left(\boldsymbol{z}, \boldsymbol{o}_{l, k}\right) \otimes \frac{\partial^{2} A\left(z, p_{l, k}\right)}{\partial\left\langle\boldsymbol{p}_{k}\right\rangle_{d_{1}} \partial\left\langle\boldsymbol{p}_{k}\right\rangle_{d_{2}}}
\end{array}\right]^{\mathrm{H}}
$$

with

$$
\begin{gathered}
\frac{\partial^{2} \boldsymbol{a}\left(\boldsymbol{z}, \boldsymbol{o}_{l, k}\right)}{\partial\left\langle\boldsymbol{p}_{k}\right\rangle_{d_{1}} \partial\left\langle\boldsymbol{p}_{k}\right\rangle_{d_{2}}}=\dot{\boldsymbol{\psi}}_{1, d_{1}}^{(b)}\left(\boldsymbol{z}, \boldsymbol{o}_{l, k}\right) \dot{\boldsymbol{\psi}}_{1, d_{2}}^{(b)}\left(\boldsymbol{z}, \boldsymbol{o}_{l, k}\right) \boldsymbol{a}\left(\boldsymbol{z}, \boldsymbol{o}_{l, k}\right)+\boldsymbol{a}\left(\boldsymbol{z}, \boldsymbol{o}_{l, k}\right) \boldsymbol{\psi}_{1, d_{1} d_{2}}\left(\boldsymbol{z}, \boldsymbol{o}_{l, k}\right) \\
. \cdot(b b) \\
\boldsymbol{\psi}_{1, d_{1} d_{2}}\left(\boldsymbol{z}, \boldsymbol{o}_{l, k}\right)=\left\langle\dot{\delta}^{\cdot(b b)}\left(\boldsymbol{z}, \boldsymbol{o}_{l, k}\right)\right\rangle_{d_{1} d_{2}} \cdot \operatorname{diag}\left\{j 2 \pi \frac{d}{\lambda} \widetilde{\boldsymbol{M}}\right\}
\end{gathered}
$$

and

$$
\frac{\partial^{2} A\left(z, p_{l, k}\right)}{\partial\left\langle p_{k}\right\rangle_{d_{1}} \partial\left\langle p_{k}\right\rangle_{d_{2}}}=A\left(z, p_{l, k}\right) \dot{\psi}_{2, d_{1} d_{2}}^{(b b)}\left(z, p_{l, k}\right)+\dot{\psi}_{2, d_{1}}^{(b)}\left(z, p_{l, k}\right) A\left(z, p_{l, k}\right) \dot{\psi}_{2, d_{2}}^{(b)}\left(z, p_{l, k}\right)
$$




$$
\ddot{\psi}_{2, d_{1} d_{2}}\left(\boldsymbol{z}, \boldsymbol{p}_{l, k}\right)=\left\langle\dot{\mu}^{(b b)}\left(\boldsymbol{z}, \boldsymbol{p}_{l, k}\right)\right\rangle_{d_{1} d_{2}} \cdot \operatorname{diag}\left\{j 2 \pi f_{c} \widetilde{\boldsymbol{N}} T_{s}\right\}
$$

.. (bb)

Therefore, $\bar{C}_{d_{1} d_{2}}\left(\boldsymbol{z}, \boldsymbol{p}_{k}\right)$ can be written as

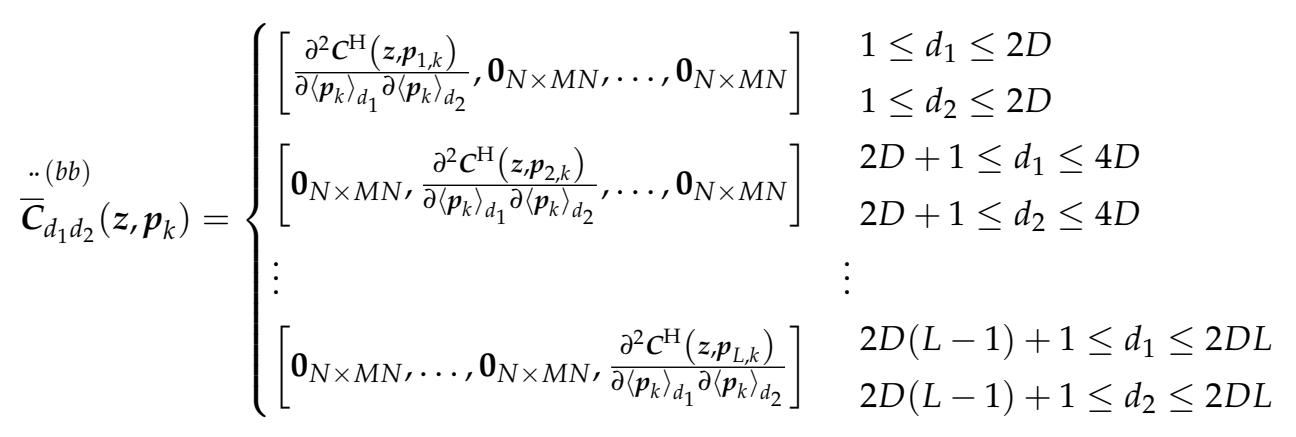

This completes the derivation.

\section{Appendix C. Derivation of (31) to (34)}

Associated with the second formulation in (21), it follows for any vector $q_{1} \in \mathbb{C}^{L \times 1}$ and $\boldsymbol{q}_{2} \in \mathbb{C}^{N \times 1}$ that

$$
\begin{aligned}
& \widetilde{\boldsymbol{D}}_{k}^{(1)} \boldsymbol{q}_{1}=\sum_{d=1}^{D}\langle\widetilde{\boldsymbol{z}}\rangle_{d} \dot{\overline{\boldsymbol{C}}}_{d}^{(a)}\left(\boldsymbol{z}, \boldsymbol{p}_{k}\right) \boldsymbol{Y}_{k} \boldsymbol{q}_{1}+\sum_{d=1}^{2 D L}\left\langle\widetilde{\boldsymbol{p}}_{k}\right\rangle_{d} \dot{\overline{\boldsymbol{C}}}_{d}^{(b)}\left(\boldsymbol{z}, \boldsymbol{p}_{k}\right) \boldsymbol{Y}_{k} \boldsymbol{q}_{1}+\overline{\boldsymbol{C}}\left(\boldsymbol{z}, \boldsymbol{p}_{k}\right) \boldsymbol{N}_{k} \boldsymbol{q}_{1} \\
& =\frac{\partial\left(\overline{\boldsymbol{C}}\left(\boldsymbol{z}, \boldsymbol{p}_{k}\right) \boldsymbol{Y}_{k} \boldsymbol{q}_{1}\right)}{\partial \boldsymbol{z}^{\mathrm{T}}} \widetilde{\boldsymbol{z}}+\frac{\partial\left(\overline{\boldsymbol{C}}\left(\boldsymbol{z}, \boldsymbol{p}_{k}\right) \boldsymbol{Y}_{k} \boldsymbol{q}_{1}\right)}{\partial \boldsymbol{p}_{k}^{\mathrm{T}}} \widetilde{\boldsymbol{p}}_{k}+\overline{\boldsymbol{C}}\left(\boldsymbol{z}, \boldsymbol{p}_{k}\right)\left(\operatorname{diag}\left\{\boldsymbol{q}_{1}\right\} \otimes \boldsymbol{I}_{N}\right) \boldsymbol{\Pi}_{1} \widetilde{\boldsymbol{n}}_{k} \\
& =\boldsymbol{F}_{1 k}^{(a)}\left(\boldsymbol{q}_{1}\right) \widetilde{\boldsymbol{z}}+\boldsymbol{F}_{2 k}^{(a)}\left(\boldsymbol{q}_{1}\right) \widetilde{\boldsymbol{p}}_{k}+\boldsymbol{F}_{3 k}^{(a)}\left(\boldsymbol{q}_{1}\right) \widetilde{\boldsymbol{n}}_{k} \\
& \widetilde{\boldsymbol{D}}_{k}^{(1) \mathrm{H}} \boldsymbol{q}_{2}=\sum_{d=1}^{D}\langle\widetilde{\boldsymbol{z}}\rangle_{d} \boldsymbol{Y}_{k}^{\mathrm{H}} \dot{\overline{\boldsymbol{C}}}_{d}^{(a) \mathrm{H}}\left(\boldsymbol{z}, \boldsymbol{p}_{k}\right) \boldsymbol{q}_{2}+\sum_{d=1}^{2 D L}\left\langle\widetilde{\mathbf{p}}_{k}\right\rangle_{d} \boldsymbol{Y}_{k}^{\mathrm{H}} \dot{\overline{\boldsymbol{C}}}_{d}^{(b) \mathrm{H}}\left(\boldsymbol{z}, \boldsymbol{p}_{k}\right) \boldsymbol{q}_{2}+\boldsymbol{N}_{k}^{\mathrm{H}} \overline{\boldsymbol{C}}\left(\boldsymbol{z}, \boldsymbol{p}_{k}\right) \boldsymbol{q}_{2} \\
& =\frac{\partial\left(\boldsymbol{Y}_{k}^{\mathrm{H}} \overline{\boldsymbol{C}}^{\mathrm{H}}\left(\boldsymbol{z}, \boldsymbol{p}_{k}\right) \boldsymbol{q}_{2}\right)}{\partial \boldsymbol{z}^{\mathrm{T}}} \widetilde{\boldsymbol{z}}+\frac{\partial\left(\boldsymbol{\gamma}_{k}^{\mathrm{H}} \overline{\boldsymbol{C}}^{\mathrm{H}}\left(\boldsymbol{z}, \boldsymbol{p}_{k}\right) \boldsymbol{q}_{2}\right)}{\partial \boldsymbol{p}_{k}^{\mathrm{T}}} \widetilde{\boldsymbol{p}}_{k}+\left(\boldsymbol{I}_{L} \otimes \boldsymbol{q}_{2}^{\mathrm{T}}\right) \overline{\overline{\boldsymbol{C}}}\left(\boldsymbol{z}, \boldsymbol{p}_{k}\right) \boldsymbol{\Pi}_{2} \widetilde{\boldsymbol{n}}_{k} \\
& =\boldsymbol{F}_{1 k}^{(b)}\left(\boldsymbol{q}_{2}\right) \widetilde{\boldsymbol{z}}+\boldsymbol{F}_{2 k}^{(b)}\left(\boldsymbol{q}_{2}\right) \widetilde{\boldsymbol{p}}_{k}+\boldsymbol{F}_{3 k}^{(b)}\left(\boldsymbol{q}_{2}\right) \widetilde{\boldsymbol{n}}_{k}
\end{aligned}
$$

Consequently, the formulation of $\widetilde{J}_{\text {cost }}^{(1)}$ can be shown as

$$
\begin{aligned}
\widetilde{J}_{\text {cost }}^{(1)}= & \sum_{k=1}^{K} \boldsymbol{\alpha}_{k, L}^{(0) \mathrm{H}} \boldsymbol{D}_{k}^{(0) \mathrm{H}}\left(\boldsymbol{F}_{1 k}^{(a)}\left(\boldsymbol{\alpha}_{k, L}^{(0)}\right) \widetilde{z}+\boldsymbol{F}_{2 k}^{(a)}\left(\boldsymbol{\alpha}_{k, L}^{(0)}\right) \widetilde{p}_{k}+\boldsymbol{F}_{3 k}^{(a)}\left(\boldsymbol{\alpha}_{k, L}^{(0)}\right) \widetilde{n}_{k}\right)+ \\
& \sum_{k=1}^{K} \boldsymbol{\alpha}_{k, L}^{(0) \mathrm{H}}\left(\boldsymbol{F}_{1 k}^{(b)}\left(\boldsymbol{D}_{k}^{(0)} \boldsymbol{\alpha}_{k, L}^{(0)}\right) \widetilde{z}+\boldsymbol{F}_{2 k}^{(b)}\left(\boldsymbol{D}_{k}^{(0)} \boldsymbol{\alpha}_{k, L}^{(0)}\right) \widetilde{p}_{k}+\boldsymbol{F}_{3 k}^{(b)}\left(\boldsymbol{D}_{k}^{(0)} \boldsymbol{\alpha}_{k, L}^{(0)}\right) \widetilde{n}_{k}\right)
\end{aligned}
$$

This completes the derivation. 


\section{Appendix D. Derivation of (35)}

Associated with (A46), it follows for any vector $\boldsymbol{q}_{1}$ and $\boldsymbol{q}_{2}$ that

$$
\begin{aligned}
\boldsymbol{q}_{1}^{\mathrm{H}} \widetilde{\boldsymbol{D}}_{k}^{(1) \mathrm{H}} \boldsymbol{\Phi} \widetilde{\boldsymbol{D}}_{k}^{(1)} \boldsymbol{q}_{2}= & \widetilde{\boldsymbol{z}}^{\mathrm{T}} \boldsymbol{F}_{1 k}^{(a) \mathrm{H}}\left(\boldsymbol{q}_{1}\right) \boldsymbol{\Phi} \boldsymbol{F}_{1 k}^{(a)}\left(\boldsymbol{q}_{2}\right) \widetilde{\boldsymbol{z}}+\widetilde{\boldsymbol{p}}_{k}^{\mathrm{T}} \boldsymbol{F}_{2 k}^{(a) \mathrm{H}}\left(\boldsymbol{q}_{1}\right) \boldsymbol{\Phi} \boldsymbol{F}_{2 k}^{(a)}\left(\boldsymbol{q}_{2}\right) \widetilde{\boldsymbol{p}}_{k}+ \\
& \widetilde{\boldsymbol{z}}^{\mathrm{T}}\left(\boldsymbol{F}_{1 k}^{(a) \mathrm{H}}\left(\boldsymbol{q}_{1}\right) \boldsymbol{\Phi} \boldsymbol{F}_{2 k}^{(a)}\left(\boldsymbol{q}_{2}\right)+\boldsymbol{F}_{1 k}^{(a) \mathrm{T}}\left(\boldsymbol{q}_{2}\right) \boldsymbol{\Phi}^{\mathrm{T}} \boldsymbol{F}_{2 k}^{(a) *}\left(\boldsymbol{q}_{1}\right)\right) \widetilde{\boldsymbol{p}}_{k}+ \\
& \widetilde{\boldsymbol{z}}^{\mathrm{T}}\left(\boldsymbol{F}_{1 k}^{(a) \mathrm{H}}\left(\boldsymbol{q}_{1}\right) \boldsymbol{\Phi} \boldsymbol{F}_{3 k}^{(a)}\left(\boldsymbol{q}_{2}\right)+\boldsymbol{F}_{1 k}^{(a) \mathrm{T}}\left(\boldsymbol{q}_{2}\right) \boldsymbol{\Phi}^{\mathrm{T}} \boldsymbol{F}_{3 k}^{(a) *}\left(\boldsymbol{q}_{1}\right) \boldsymbol{\Pi}_{3}\right) \widetilde{\boldsymbol{n}}_{k}+ \\
& \widetilde{\boldsymbol{p}}_{k}^{\mathrm{T}}\left(\boldsymbol{F}_{2 k}^{(a) \mathrm{H}}\left(\boldsymbol{q}_{1}\right) \boldsymbol{\Phi} \boldsymbol{F}_{3 k}^{(a)}\left(\boldsymbol{q}_{2}\right)+\boldsymbol{F}_{2 k}^{(a) \mathrm{T}}\left(\boldsymbol{q}_{2}\right) \boldsymbol{\Phi}^{\mathrm{T}} \boldsymbol{F}_{3 k}^{(a) *}\left(\boldsymbol{q}_{1}\right) \boldsymbol{\Pi}_{3}\right) \widetilde{\boldsymbol{n}}_{k}+ \\
& \widetilde{\boldsymbol{n}}_{k}^{\mathrm{T}} \boldsymbol{F}_{3 k}^{(a) \mathrm{H}}\left(\boldsymbol{q}_{1}\right) \boldsymbol{\Phi} \boldsymbol{F}_{3 k}^{(a)}\left(\boldsymbol{q}_{2}\right) \widetilde{\boldsymbol{n}}_{k} \\
= & \widetilde{\boldsymbol{z}}^{\mathrm{T}} \boldsymbol{\Sigma}_{1 k}^{(a)}\left(\boldsymbol{q}_{1}, \boldsymbol{\Phi}, \boldsymbol{q}_{2}\right) \widetilde{\boldsymbol{z}}+\widetilde{\boldsymbol{p}}_{k}^{\mathrm{T}} \boldsymbol{\Sigma}_{2 k}^{(a)}\left(\boldsymbol{q}_{1}, \boldsymbol{\Phi}, \boldsymbol{q}_{2}\right) \widetilde{\boldsymbol{p}}_{k}+ \\
& \widetilde{\boldsymbol{z}}^{\mathrm{T}} \boldsymbol{\Sigma}_{3 k}^{(a)}\left(\boldsymbol{q}_{1}, \boldsymbol{\Phi}, \boldsymbol{q}_{2}\right) \widetilde{\boldsymbol{p}}_{k}+\widetilde{\boldsymbol{z}}^{\mathrm{T}} \boldsymbol{\Sigma}_{4 k}^{(a)}\left(\boldsymbol{q}_{1}, \boldsymbol{\Phi}, \boldsymbol{q}_{2}\right) \widetilde{\boldsymbol{n}}_{k}+ \\
& \widetilde{\boldsymbol{p}}_{k}^{\mathrm{T}} \boldsymbol{\Sigma}_{5 k}^{(a)}\left(\boldsymbol{q}_{1}, \boldsymbol{\Phi}, \boldsymbol{q}_{2}\right) \widetilde{\boldsymbol{n}}_{k}+\widetilde{\boldsymbol{n}}_{k}^{\mathrm{T}} \boldsymbol{\Sigma}_{6 k}^{(a)}\left(\boldsymbol{q}_{1}, \boldsymbol{\Phi}, \boldsymbol{q}_{2}\right) \widetilde{\boldsymbol{n}}_{k}
\end{aligned}
$$

Meanwhile, the similar result respect to (A47) is drawn as

$$
\begin{aligned}
\boldsymbol{q}_{1}^{\mathrm{H}} \widetilde{\boldsymbol{D}}_{k}^{(1)} \boldsymbol{\Phi} \widetilde{\boldsymbol{D}}_{k}^{(1) \mathrm{H}} \boldsymbol{q}_{2}= & \widetilde{\boldsymbol{z}}^{\mathrm{T}} \boldsymbol{F}_{1 k}^{(b) \mathrm{H}}\left(\boldsymbol{q}_{1}\right) \boldsymbol{\Phi} \boldsymbol{F}_{1 k}^{(b)}\left(\boldsymbol{q}_{2}\right) \widetilde{\boldsymbol{z}}+\widetilde{\boldsymbol{p}}_{k}^{\mathrm{T}} \boldsymbol{F}_{2 k}^{(b) \mathrm{H}}\left(\boldsymbol{q}_{1}\right) \boldsymbol{\Phi} \boldsymbol{F}_{2 k}^{(b)}\left(\boldsymbol{q}_{2}\right) \widetilde{\boldsymbol{p}}_{k}+ \\
& \widetilde{\boldsymbol{z}}^{\mathrm{T}}\left(\boldsymbol{F}_{1 k}^{(b) \mathrm{H}}\left(\boldsymbol{q}_{1}\right) \boldsymbol{\Phi} \boldsymbol{F}_{2 k}^{(b)}\left(\boldsymbol{q}_{2}\right)+\boldsymbol{F}_{1 k}^{(b) \mathrm{T}}\left(\boldsymbol{q}_{2}\right) \boldsymbol{\Phi}^{\mathrm{T}} \boldsymbol{F}_{2 k}^{(b) *}\left(\boldsymbol{q}_{1}\right)\right) \widetilde{\boldsymbol{p}}_{k}+ \\
& \widetilde{\boldsymbol{z}}^{\mathrm{T}}\left(\boldsymbol{F}_{1 k}^{(b) \mathrm{H}}\left(\boldsymbol{q}_{1}\right) \boldsymbol{\Phi} \boldsymbol{F}_{3 k}^{(b)}\left(\boldsymbol{q}_{2}\right)+\boldsymbol{F}_{1 k}^{(b) \mathrm{T}}\left(\boldsymbol{q}_{2}\right) \boldsymbol{\Phi}^{\mathrm{T}} \boldsymbol{F}_{3 k}^{(b) *}\left(\boldsymbol{q}_{1}\right) \boldsymbol{\Pi}_{3}\right) \widetilde{\boldsymbol{n}}_{k}+ \\
& \widetilde{\boldsymbol{p}}_{k}^{\mathrm{T}}\left(\boldsymbol{F}_{2 k}^{(b) \mathrm{H}}\left(\boldsymbol{q}_{1}\right) \boldsymbol{\Phi} \boldsymbol{F}_{3 k}^{(b)}\left(\boldsymbol{q}_{2}\right)+\boldsymbol{F}_{2 k}^{(b) \mathrm{T}}\left(\boldsymbol{q}_{2}\right) \boldsymbol{\Phi}^{\mathrm{T}} \boldsymbol{F}_{3 k}^{(b) *}\left(\boldsymbol{q}_{1}\right) \boldsymbol{\Pi}_{3}\right) \widetilde{\boldsymbol{n}}_{k}+ \\
& \widetilde{\boldsymbol{n}}_{k}^{\mathrm{T}} \boldsymbol{F}_{3 k}^{(b) \mathrm{H}}\left(\boldsymbol{q}_{1}\right) \boldsymbol{\Phi} \boldsymbol{F}_{3 k}^{(b)}\left(\boldsymbol{q}_{2}\right) \widetilde{\boldsymbol{n}}_{k} \\
= & \widetilde{\boldsymbol{z}}^{\mathrm{T}} \boldsymbol{\Sigma}_{1 k}^{(b)}\left(\boldsymbol{q}_{1}, \boldsymbol{\Phi}, \boldsymbol{q}_{2}\right) \widetilde{\boldsymbol{z}}+\widetilde{\boldsymbol{p}}_{k}^{\mathrm{T}} \boldsymbol{\Sigma}_{2 k}^{(b)}\left(\boldsymbol{q}_{1}, \boldsymbol{\Phi}, \boldsymbol{q}_{2}\right) \widetilde{\boldsymbol{p}}_{k}+ \\
& \widetilde{\boldsymbol{z}}^{\mathrm{T}} \boldsymbol{\Sigma}_{3 k}^{(b)}\left(\boldsymbol{q}_{1}, \boldsymbol{\Phi}, \boldsymbol{q}_{2}\right) \widetilde{\boldsymbol{p}}_{k}+\widetilde{\boldsymbol{z}}^{\mathrm{T}} \boldsymbol{\Sigma}_{4 k}^{(b)}\left(\boldsymbol{q}_{1}, \boldsymbol{\Phi}, \boldsymbol{q}_{2}\right) \widetilde{\boldsymbol{n}}_{k}+ \\
& \widetilde{\boldsymbol{p}}_{k}^{\mathrm{T}} \boldsymbol{\Sigma}_{5 k}^{(b)}\left(\boldsymbol{q}_{1}, \boldsymbol{\Phi}, \boldsymbol{q}_{2}\right) \widetilde{\boldsymbol{n}}_{k}+\widetilde{\boldsymbol{n}}_{k}^{\mathrm{T}} \boldsymbol{\Sigma}_{6 k}^{(b)}\left(\boldsymbol{q}_{1}, \boldsymbol{\Phi}, \boldsymbol{q}_{2}\right) \widetilde{\boldsymbol{n}}_{k}
\end{aligned}
$$

Additionally, we can obtain the following formulations

$$
\begin{aligned}
& \boldsymbol{q}_{1}^{\mathrm{H}} \widetilde{\boldsymbol{D}}_{k}^{(1)} \boldsymbol{\Phi} \widetilde{\boldsymbol{D}}_{k}^{(1)} \boldsymbol{q}_{2}=\widetilde{\boldsymbol{z}}^{\mathrm{T}} \boldsymbol{F}_{1 k}^{(b) \mathrm{H}}\left(\boldsymbol{q}_{1}\right) \boldsymbol{\Phi} \boldsymbol{F}_{1 k}^{(a)}\left(\boldsymbol{q}_{2}\right) \widetilde{\boldsymbol{z}}+\widetilde{\boldsymbol{p}}_{k}^{\mathrm{T}} \boldsymbol{F}_{2 k}^{(b) \mathrm{H}}\left(\boldsymbol{q}_{1}\right) \boldsymbol{\Phi} \boldsymbol{F}_{2 k}^{(a)}\left(\boldsymbol{q}_{2}\right) \widetilde{\boldsymbol{p}}_{k}+ \\
& \widetilde{\boldsymbol{z}}^{\mathrm{T}}\left(\boldsymbol{F}_{1 k}^{(b) \mathrm{H}}\left(\boldsymbol{q}_{1}\right) \boldsymbol{\Phi} \boldsymbol{F}_{2 k}^{(a)}\left(\boldsymbol{q}_{2}\right)+\boldsymbol{F}_{1 k}^{(a) \mathrm{T}}\left(\boldsymbol{q}_{2}\right) \boldsymbol{\Phi}^{\mathrm{T}} \boldsymbol{F}_{2 k}^{(b) *}\left(\boldsymbol{q}_{1}\right)\right) \widetilde{\boldsymbol{p}}_{k}+ \\
& \widetilde{\boldsymbol{z}}^{\mathrm{T}}\left(\boldsymbol{F}_{1 k}^{(b) \mathrm{H}}\left(\boldsymbol{q}_{1}\right) \boldsymbol{\Phi} \boldsymbol{F}_{3 k}^{(a)}\left(\boldsymbol{q}_{2}\right)+\boldsymbol{F}_{1 k}^{(a) \mathrm{T}}\left(\boldsymbol{q}_{2}\right) \boldsymbol{\Phi}^{\mathrm{T}} \boldsymbol{F}_{3 k}^{(b) *}\left(\boldsymbol{q}_{1}\right) \boldsymbol{\Pi}_{3}\right) \widetilde{\boldsymbol{n}}_{k}+ \\
& \widetilde{\boldsymbol{p}}_{k}^{\mathrm{T}}\left(\boldsymbol{F}_{2 k}^{(b) \mathrm{H}}\left(\boldsymbol{q}_{1}\right) \boldsymbol{\Phi} \boldsymbol{F}_{3 k}^{(b)}\left(\boldsymbol{q}_{2}\right)+\boldsymbol{F}_{2 k}^{(a) \mathrm{T}}\left(\boldsymbol{q}_{2}\right) \boldsymbol{\Phi}^{\mathrm{T}} \boldsymbol{F}_{3 k}^{(b) *}\left(\boldsymbol{q}_{1}\right) \boldsymbol{\Pi}_{3}\right) \widetilde{\boldsymbol{n}}_{k}+ \\
& \widetilde{\boldsymbol{n}}_{k}^{\mathrm{T}} \boldsymbol{F}_{3 k}^{(b) \mathrm{H}}\left(\boldsymbol{q}_{1}\right) \boldsymbol{\Phi} \boldsymbol{F}_{3 k}^{(a)}\left(\boldsymbol{q}_{2}\right) \widetilde{\boldsymbol{n}}_{k} \\
& =\widetilde{\boldsymbol{z}}^{\mathrm{T}} \boldsymbol{\Sigma}_{1 k}^{(c)}\left(\boldsymbol{q}_{1}, \boldsymbol{\Phi}, \boldsymbol{q}_{2}\right) \widetilde{\boldsymbol{z}}+\widetilde{\boldsymbol{p}}_{k}^{\mathrm{T}} \boldsymbol{\Sigma}_{2 k}^{(c)}\left(\boldsymbol{q}_{1}, \boldsymbol{\Phi}, \boldsymbol{q}_{2}\right) \widetilde{\boldsymbol{p}}_{k}+ \\
& \widetilde{\boldsymbol{z}}^{\mathrm{T}} \boldsymbol{\Sigma}_{3 k}^{(c)}\left(\boldsymbol{q}_{1}, \boldsymbol{\Phi}, \boldsymbol{q}_{2}\right) \widetilde{\boldsymbol{p}}_{k}+\widetilde{\boldsymbol{z}}^{\mathrm{T}} \boldsymbol{\Sigma}_{4 k}^{(c)}\left(\boldsymbol{q}_{1}, \boldsymbol{\Phi}, \boldsymbol{q}_{2}\right) \widetilde{\boldsymbol{n}}_{k}+ \\
& \widetilde{\boldsymbol{p}}_{k}^{\mathrm{T}} \boldsymbol{\Sigma}_{5 k}^{(c)}\left(\boldsymbol{q}_{1}, \boldsymbol{\Phi}, \boldsymbol{q}_{2}\right) \widetilde{\boldsymbol{n}}_{k}+\widetilde{\boldsymbol{n}}_{k}^{\mathrm{T}} \boldsymbol{\Sigma}_{6 k}^{(c)}\left(\boldsymbol{q}_{1}, \boldsymbol{\Phi}, \boldsymbol{q}_{2}\right) \widetilde{\boldsymbol{n}}_{k} \\
& \boldsymbol{q}_{1}^{\mathrm{H}} \widetilde{\boldsymbol{D}}_{k}^{(1) \mathrm{H}} \boldsymbol{\Phi} \widetilde{\boldsymbol{D}}_{k}^{(1) \mathrm{H}} \boldsymbol{q}_{2}=\left(\boldsymbol{q}_{2}^{\mathrm{H}} \widetilde{\boldsymbol{D}}_{k}^{(1)} \boldsymbol{\Phi}^{\mathrm{H}} \widetilde{\boldsymbol{D}}_{k}^{(1)} \boldsymbol{q}_{1}\right)^{\mathrm{H}} \\
& =\widetilde{\boldsymbol{z}}^{\mathrm{T}} \boldsymbol{\Sigma}_{1 k}^{(c) *}\left(\boldsymbol{q}_{2}, \boldsymbol{\Phi}^{\mathrm{H}}, \boldsymbol{q}_{1}\right) \widetilde{\boldsymbol{z}}+\widetilde{\boldsymbol{p}}_{k}^{\mathrm{T}} \boldsymbol{\Sigma}_{2 k}^{(c) *}\left(\boldsymbol{q}_{2}, \boldsymbol{\Phi}^{\mathrm{H}}, \boldsymbol{q}_{1}\right) \widetilde{\boldsymbol{p}}_{k}+ \\
& \widetilde{\boldsymbol{z}}^{\mathrm{T}} \boldsymbol{\Sigma}_{3 k}^{(c) *}\left(\boldsymbol{q}_{2}, \boldsymbol{\Phi}^{\mathrm{H}}, \boldsymbol{q}_{1}\right) \widetilde{\boldsymbol{p}}_{k}+\widetilde{\boldsymbol{z}}^{\mathrm{T}} \boldsymbol{\Sigma}_{4 k}^{(c) *}\left(\boldsymbol{q}_{2}, \boldsymbol{\Phi}^{\mathrm{H}}, \boldsymbol{q}_{1}\right) \boldsymbol{\Pi}_{3} \widetilde{\boldsymbol{n}}_{k}+ \\
& \widetilde{\boldsymbol{p}}_{k}^{\mathrm{T}} \boldsymbol{\Sigma}_{5 k}^{(c) *}\left(\boldsymbol{q}_{2}, \boldsymbol{\Phi}^{\mathrm{H}}, \boldsymbol{q}_{1}\right) \boldsymbol{\Pi}_{3} \widetilde{\boldsymbol{n}}_{k}+\widetilde{\boldsymbol{n}}_{k}^{\mathrm{T}} \boldsymbol{\Sigma}_{6 k}^{(c) \mathrm{H}}\left(\boldsymbol{q}_{2}, \boldsymbol{\Phi}^{\mathrm{H}}, \boldsymbol{q}_{1}\right) \widetilde{\boldsymbol{n}}_{k}
\end{aligned}
$$


Finally, associated with the third formulation in (21), we have

$$
\begin{aligned}
\boldsymbol{q}_{1}^{\mathrm{H}} \widetilde{\boldsymbol{D}}_{k}^{(2)} \boldsymbol{q}_{2}= & \frac{1}{2} \sum_{d_{1}=1}^{D} \sum_{d_{2}=1}^{D}\langle\widetilde{\boldsymbol{z}}\rangle_{d_{1}}\langle\widetilde{\boldsymbol{z}}\rangle_{d_{2}} \boldsymbol{q}_{1}^{\mathrm{H}} \ddot{\overline{\boldsymbol{C}}}_{d_{1} d_{2}}^{(a a)}\left(\boldsymbol{z}, \boldsymbol{p}_{k}\right) \boldsymbol{y}_{k} \boldsymbol{q}_{2}+ \\
& \frac{1}{2} \sum_{d_{1}=1}^{2 D L} \sum_{d_{2}=1}^{2 D L}\left\langle\widetilde{\boldsymbol{p}}_{k}\right\rangle_{d_{1}}\left\langle\widetilde{\boldsymbol{p}}_{k}\right\rangle_{d_{2}} \boldsymbol{q}_{1}^{\mathrm{H}} \ddot{\overline{\boldsymbol{C}}}_{d_{1} d_{2}}(b b) \\
& \sum^{D} \sum^{2 D L}\left\langle\widetilde{\boldsymbol{z}_{k}}\right) \boldsymbol{\boldsymbol { q }}_{k} \boldsymbol{q}_{d_{1}}\left\langle\widetilde{\boldsymbol{p}}_{k}\right\rangle_{d_{2}} \boldsymbol{q}_{1}^{\mathrm{H}} \ddot{\overline{\boldsymbol{C}}}_{d_{1} d_{2}}^{(a b)}\left(\boldsymbol{z}, \boldsymbol{p}_{k}\right) \boldsymbol{Y}_{k} \boldsymbol{q}_{2}+ \\
& d_{1}=1 d_{2}=1 \\
& \sum_{d=1}^{D}\langle\widetilde{\boldsymbol{z}}\rangle_{d} \boldsymbol{q}_{1}^{\mathrm{H}} \dot{\overline{\boldsymbol{C}}}_{d}^{(a)}\left(\boldsymbol{z}, \boldsymbol{p}_{k}\right) \boldsymbol{N}_{k} \boldsymbol{q}_{2}+\sum_{d=1}^{2 D L}\left\langle\widetilde{\boldsymbol{p}}_{k}\right\rangle_{d} \boldsymbol{q}_{1}^{\mathrm{H}} \dot{\overline{\boldsymbol{C}}}_{d}^{(b)}\left(\boldsymbol{z}, \boldsymbol{p}_{k}\right) \boldsymbol{N}_{k} \boldsymbol{q}_{2} \\
= & \widetilde{\boldsymbol{z}}^{\mathrm{T}} \boldsymbol{\Sigma}_{1 k}^{(d)}\left(\boldsymbol{q}_{1}, \boldsymbol{q}_{2}\right) \widetilde{\boldsymbol{z}}+\widetilde{\boldsymbol{p}}_{k}^{\mathrm{T}} \boldsymbol{\Sigma}_{2 k}^{(d)}\left(\boldsymbol{q}_{1}, \boldsymbol{q}_{2}\right) \widetilde{\boldsymbol{p}}_{k}+\widetilde{\boldsymbol{z}}^{\mathrm{T}} \boldsymbol{\Sigma}_{3 k}^{(d)}\left(\boldsymbol{q}_{1}, \boldsymbol{q}_{2}\right) \widetilde{\boldsymbol{p}}_{k}+ \\
& \widetilde{\boldsymbol{z}}^{\mathrm{T}} \boldsymbol{\Sigma}_{4 k}^{(d)}\left(\boldsymbol{q}_{1}, \boldsymbol{q}_{2}\right) \widetilde{\boldsymbol{n}}_{k}+\widetilde{\boldsymbol{p}}_{k}^{\mathrm{T}} \boldsymbol{\Sigma}_{5 k}^{(d)}\left(\boldsymbol{q}_{1}, \boldsymbol{q}_{2}\right) \widetilde{\boldsymbol{n}}_{k} \\
\boldsymbol{q}_{1}^{\mathrm{H}} \widetilde{\boldsymbol{D}}_{k}^{(2) \mathrm{H}} \boldsymbol{q}_{2}= & \left(\boldsymbol{q}_{2}^{\mathrm{H}} \widetilde{\boldsymbol{D}}_{k}^{(2)} \boldsymbol{q}_{1}\right) \\
= & \widetilde{\boldsymbol{z}}^{\mathrm{T}} \boldsymbol{\Sigma}_{1 k}^{(d) *}\left(\boldsymbol{q}_{2}, \boldsymbol{q}_{1}\right) \widetilde{\boldsymbol{z}}+\widetilde{\boldsymbol{p}}_{k}^{\mathrm{T}} \boldsymbol{\Sigma}_{2 k}^{(d) *}\left(\boldsymbol{q}_{2}, \boldsymbol{q}_{1}\right) \widetilde{\boldsymbol{p}}_{k}+\widetilde{\boldsymbol{z}}^{\mathrm{T}} \boldsymbol{\Sigma}_{3 k}^{(d) *}\left(\boldsymbol{q}_{2}, \boldsymbol{q}_{1}\right) \widetilde{\boldsymbol{p}}_{k}+ \\
& \widetilde{\boldsymbol{z}}^{\mathrm{T}} \boldsymbol{\Sigma}_{4 k}^{(d) *}\left(\boldsymbol{q}_{2}, \boldsymbol{q}_{1}\right) \boldsymbol{\Pi}_{3} \widetilde{\boldsymbol{n}}_{k}+\widetilde{\boldsymbol{p}}_{k}^{\mathrm{T}} \boldsymbol{\Sigma}_{5 k}^{(d) *}\left(\boldsymbol{q}_{2}, \boldsymbol{q}_{1}\right) \boldsymbol{\Pi}_{3} \widetilde{\boldsymbol{n}}_{k}
\end{aligned}
$$

By using the above results, the expression of $\widetilde{J}_{\text {cost }}^{(2)}$ is written as

$$
\begin{aligned}
\widetilde{J}_{\text {cost }}^{(2)}= & \sum_{k=1}^{K} \widetilde{z}^{\mathrm{T}} \boldsymbol{\xi}_{1 k} \widetilde{z}+\sum_{k=1}^{K} \widetilde{p}_{k}^{\mathrm{T}} \boldsymbol{\xi}_{2 k} \widetilde{p}_{k}+\sum_{k=1}^{K} \widetilde{z}^{\mathrm{T}} \boldsymbol{\xi}_{3 k} \widetilde{p}_{k}+ \\
& \sum_{k=1}^{K} \widetilde{z}^{\mathrm{T}} \boldsymbol{\xi}_{4 k} \widetilde{n}_{k}+\sum_{k=1}^{K} \widetilde{p}_{k}^{\mathrm{T}} \boldsymbol{\xi}_{5 k} \widetilde{n}_{k}+\sum_{k=1}^{K} \widetilde{n}_{k}^{\mathrm{H}} \boldsymbol{\xi}_{6 k} \widetilde{n}_{k}
\end{aligned}
$$

This ends the derivation in this part.

\section{Appendix E. Derivation of CRB}

Appendix E.1. The Partial of $\boldsymbol{d}_{l, k}(\boldsymbol{\eta})$ Respect to $\boldsymbol{z}$

Define $\boldsymbol{G}_{l, k}=\boldsymbol{b}_{l, k} \dot{\boldsymbol{C}}\left(z, \boldsymbol{p}_{l, k}\right)\left(\boldsymbol{I}_{2} \otimes \boldsymbol{B}_{k} \boldsymbol{s}_{k}\right)$ with $\dot{\boldsymbol{C}}\left(z, \boldsymbol{p}_{l, k}\right)=\frac{\partial C\left(z, p_{l, k}\right)}{\partial z^{\mathrm{T}}}=\frac{\partial a\left(z, p_{l, k}\right)}{\partial z^{\mathrm{T}}} \otimes$ $A\left(z, p_{l, k}\right)+a\left(z, o_{l, k}\right) \otimes \frac{\partial A\left(z, p_{l, k}\right)}{\partial z^{l}}$. The derivation with respect to $z$ can be expressed by

$$
\frac{\partial d_{l, k}(\boldsymbol{\eta})}{\partial z^{\mathrm{T}}}=G_{l, k}
$$

where

$$
\begin{aligned}
& \frac{\partial \boldsymbol{a}\left(\boldsymbol{z}, \boldsymbol{p}_{l, k}\right)}{\partial \boldsymbol{z}^{\mathrm{T}}}=\operatorname{diag}\left\{\boldsymbol{a}\left(\boldsymbol{z}, \boldsymbol{o}_{l, k}\right)\right\} \cdot\left[0, j 2 \pi \frac{d}{\lambda}, \ldots, j 2 \pi \frac{d}{\lambda}(M-1)\right]^{\mathrm{T}} \cdot \dot{\boldsymbol{\delta}}^{(a) \mathrm{T}}\left(\boldsymbol{z}, \boldsymbol{o}_{l, k}\right) \\
& \frac{\partial A\left(z, \boldsymbol{p}_{l, k}\right)}{\partial \boldsymbol{z}^{\mathrm{T}}}= A\left(\boldsymbol{z}, \boldsymbol{p}_{l, k}\right) \cdot \\
& \operatorname{diag}\left\{0, j 2 \pi f_{c} T_{s} \dot{\boldsymbol{\mu}}^{(a) \mathrm{T}}\left(\boldsymbol{z}, \boldsymbol{p}_{l, k}\right), \ldots, j 2 \pi f_{c}(N-1) T_{s} \dot{\boldsymbol{\mu}}^{(a) \mathrm{T}}\left(\boldsymbol{z}, \boldsymbol{p}_{l, k}\right)\right\}
\end{aligned}
$$

Appendix E.2. The Partial of $\boldsymbol{d}_{l, k}(\boldsymbol{\eta})$ Respect to $\boldsymbol{b}$

Define $\boldsymbol{H}_{l, k}=\boldsymbol{C}\left(\boldsymbol{z}, \boldsymbol{p}_{l, k}\right) \boldsymbol{B}_{k} \boldsymbol{s}_{k} \delta_{l, m} \delta_{k, n}$. Note that $\boldsymbol{b}$ is a complex vector, so we obtain

$$
\begin{aligned}
\frac{\partial \boldsymbol{d}_{l, k}(\boldsymbol{\eta})}{\partial \boldsymbol{b}_{m, n}^{(R \mathrm{~T}}} & =\boldsymbol{H}_{l, k} \\
\frac{\partial \boldsymbol{d}_{l, k}(\boldsymbol{\eta})}{\partial \boldsymbol{b}_{m, n}^{(I) \mathrm{T}}} & =j \boldsymbol{H}_{l, k}
\end{aligned}
$$


Appendix E.3. The Partial of $\boldsymbol{d}_{l, k}(\boldsymbol{\eta})$ Respect to $\boldsymbol{s}$

Define $\boldsymbol{K}_{l, k}=b_{l, k} \boldsymbol{C}\left(\boldsymbol{z}, \boldsymbol{p}_{l, k}\right) \boldsymbol{B}_{k} \delta_{k, n}$. Note that $\boldsymbol{s}$ is also a complex vector, so we obtain

$$
\begin{aligned}
\frac{\partial d_{l, k}(\boldsymbol{\eta})}{\partial s_{n}^{(R) \mathrm{T}}} & =\boldsymbol{K}_{l, k} \\
\frac{\partial \boldsymbol{d}_{l, k}(\boldsymbol{\eta})}{\partial \boldsymbol{s}_{n}^{(I) \mathrm{T}}} & =j \boldsymbol{K}_{l, k}
\end{aligned}
$$

Appendix E.4. The Partial of $\boldsymbol{d}_{l, k}(\boldsymbol{\eta})$ Respect to $\Delta \boldsymbol{f}$

Define $\boldsymbol{M}_{l, k}=\boldsymbol{b}_{l, k} \boldsymbol{C}\left(\boldsymbol{z}, \boldsymbol{p}_{l, k}\right) \dot{\boldsymbol{B}}_{k}\left(\boldsymbol{I}_{K} \otimes \boldsymbol{s}_{k}\right)$ with $\dot{\boldsymbol{B}}_{k}=\boldsymbol{B}_{k} \cdot \operatorname{diag}\left\{j 2 \pi \widetilde{N} T_{s}\right\} \delta_{k, n}$. The derivation with respect to $\Delta f$ can be expressed by

$$
\frac{\partial d_{l, k}(\eta)}{\partial \Delta f^{T}}=M_{l, k}
$$

By substituting (A56), (A59), (A60), (A61) into (53), the sub-blocks of $J_{\eta \eta}$ is formulated as

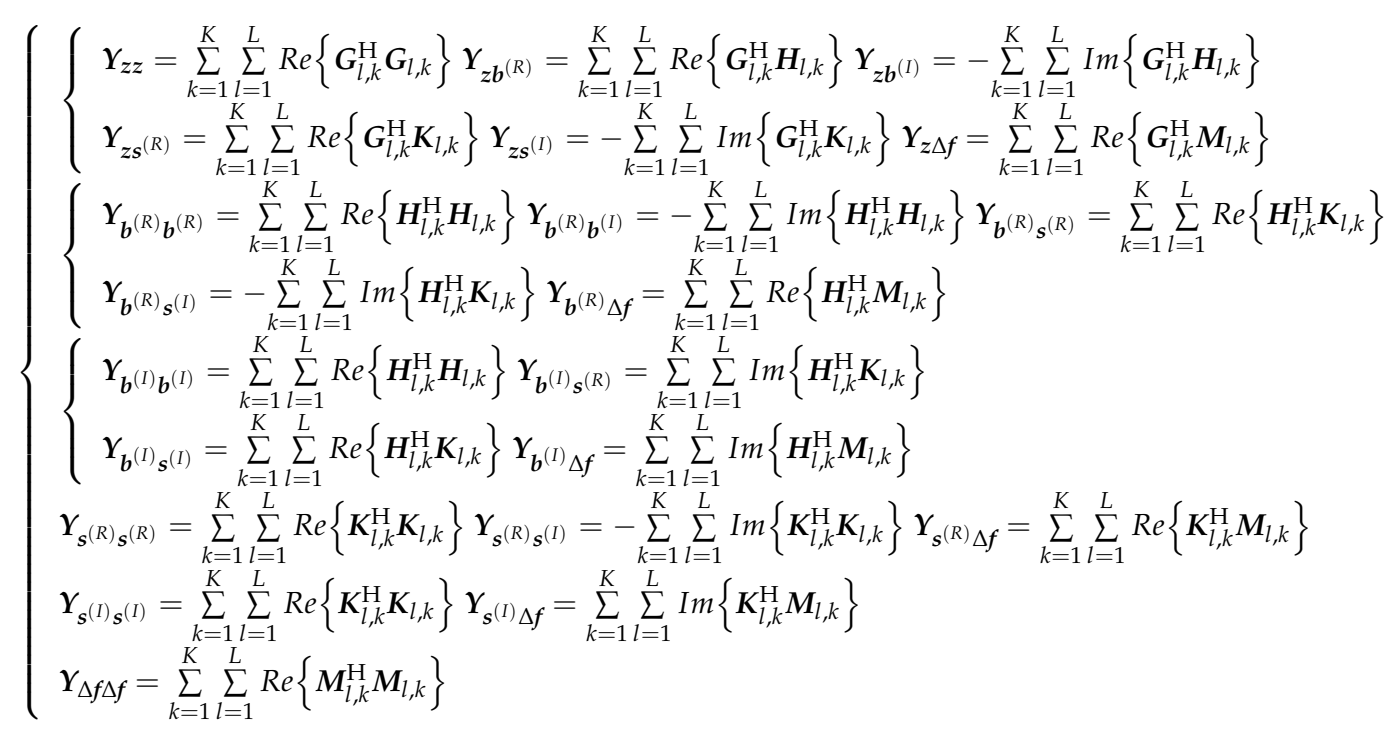

\section{References}

1. Zhang, Y.; Xu, X.; Sheikh, Y.A.; Ye, Z. A rank-reduction based 2-D DOA estimation algorithm for three parallel uniform linear arrays. Signal Process. 2016, 120, 305-310. [CrossRef]

2. Oh, D.; Kim, S.; Yoon, S.H.; Chong, J.W. Two-Dimensional ESPRIT-Like Shift-Invariant TOA Estimation Algorithm Using Multi-Band Chirp Signals Robust to Carrier Frequency Offset. IEEE Trans. Wirel. Common. 2013, 12, 3130-3139. [CrossRef]

3. Cao, H.; Chan, Y.T.; So, H.C. Maximum likelihood TDOA estimation from compressed sensing samples without reconstruction. IEEE Signal Process. Lett. 2017, 24, 564-568. [CrossRef]

4. Tahat, A.; Kaddoum, G.; Yousefi, S.; Valaee, S.; Gagnon, F. A look at the recent wireless positioning techniques with a focus on algorithms for moving receivers. IEEE Access 2016, 4, 6652-6680. [CrossRef]

5. Gajewski, P.; Ziolkowski, C.; Kelner, J.M. Using SDF method for simultaneous location of multiple radio transmitters. In Proceedings of the IEEE 19th International Conference on Microwave Radar and Wireless Communications (MIKON), Warsaw, Poland, 21-23 May 2012; Volume 2, pp. 634-637.

6. Kelner, J.M.; Ziolkowski, C.; Nowosielski, L.; Wnuk, M. Localization of emission source in urban environment based on the Doppler effect. In Proceedings of the 2016 IEEE 83rd Vehicular Technology Conference (VTC Spring), Nanjing, China, 15-18 May 2016; pp. 1-5.

7. Li, J.; Guo, F.; Yang, L.; Jiang, W.; Pang, H. On the use of calibration sensors in source localization using TDOA and FDOA measurements. Digit. Signal Process. 2014, 27, 33-43. [CrossRef] 
8. Luo, X.; Jiu, B.; Chen, S.; Ge, Q. ML estimation of transition probabilities for an unknown maneuvering emitter tracking. Signal Process. 2015, 109, 248-260. [CrossRef]

9. Viberg, M.; Ottersten, B. Sensor array processing based on subspace fitting. IEEE Trans. Signal Process. 1991, 39, 1110-1121. [CrossRef]

10. Amar, A.; Weiss, A.J. Localization of narrowband radio emitters based on Doppler frequency shifts. IEEE Trans. Signal Process. 2008, 56, 5500-5508. [CrossRef]

11. Tier, T.; Weiss, A.J. High resolution localization of narrowband radio emitters based on doppler frequency shifts. Signal Process. 2017, 141, 288-298. [CrossRef]

12. Weiss, A.J. Direct geolocation of wideband emitters based on delay and Doppler. IEEE Trans. Signal Process. 2011, 59, 2513-2521. [CrossRef]

13. Qin, T.; Li, L.; Ba, B.; Wang, D. A fast ML-based single-step localization using EM algorithm based on time delay and Doppler shift for a far-field scenario. Sensors 2018, 18, 4139. [CrossRef] [PubMed]

14. Weiss, A.J. Direct position determination of narrowband radio frequency transmitters. IEEE Signal Process. Lett. 2004, 11, 513-516. [CrossRef]

15. Du, J.; Wang, D.; Yu, W.; Yu, H.; Du, J.; Wang, D.; Yu, W.; Yu, H. Direct position determination of unknown signals in the presence of multipath propagation. Sensors 2018, 18, 892.

16. Wang, D.; Zhang, G.; Shen, C.; Zhang, J. A direct position determination algorithm for constant modulus signals with single moving observer. Acta Aeronaut. Astronaut. Sin. 2016, 37, 1622-1633.

17. Lu, Z.; Wang, J.; Ba, B.; Wang, D. A novel direct position determination algorithm for orthogonal frequency division multiplexing signals based on the time and angle of arrival. IEEE Access. 2017, 5, 25312-25321. [CrossRef]

18. Reuven, A.M.; Weiss, A.J. Direct position determination of cyclostationary signals. Signal Process. 2009, 89, 2448-2464. [CrossRef]

19. Cherchar, A.; Thameri, M.; Belouchrani, A. Performance improvement of direction finding algorithms in non-homogeneous environment through data fusion. Digit. Signal Process. 2015, 41, 41-47. [CrossRef]

20. Vincent, F.; Besson, O.; Chaumette, E. Approximate maximum likelihood estimation of two closely spaced sources. Signal Process. 2014, 97, 83-90. [CrossRef]

21. Hu, D.; Huang, Z.; Zhang, S.; Lu, J. Joint TDOA, FDOA and differential Doppler rate estimation: Method and its performance analysis. Chin. J Aeronaut. 2018, 31, 137-147. [CrossRef]

22. Hari, K.V.S.; Gummadavelli, U. Effect of spatial smoothing on the performance of subspace methods in the presence of array model errors. Automatica 1994, 30, 11-26. [CrossRef]

23. Amar, A.; Weiss, A.J. Direct position determination in the presence of model errors-known waveforms. Digit. Signal Process. 2006, 16, 52-83. [CrossRef]

24. Amar, A.; Weiss, A.J. Analysis of direct position determination approach in the presence of model errors. In Proceedings of the IEEE Convention on Electrical and Electronics Engineers, Telaviv, Israel, 6-7 September 2004; pp. 408-411.

25. Wang, D.; Yin, J.; Liu, R.; Yu, H.; Wang, Y. Performance analysis and improvement of direct position determination based on Doppler frequency shifts in presence of model errors: Case of known waveforms. Multidimens. Syst. Sign Process. 2018, 1-42. [CrossRef]

26. Wang, D.; Yu, H.; Wu, Z.; Wang, C. Performance Analysis of the Direct Position Determination Method in the Presence of Array Model Errors. Sensors 2017, 17, 1550. [CrossRef] [PubMed]

27. Tirer, T.; Weiss, A.J. Performance Analysis of a High-Resolution Direct Position Determination Method. IEEE Trans. Signal Process. 2016, 65, 544-554. [CrossRef]

28. Li, J.; Yang, L.; Guo, F.; Jiang, W. Coherent summation of multiple short-time signals for direct positioning of a wideband source based on delay and Doppler. Digit. Signal Process. 2016, 48, 58-70. [CrossRef]

29. Wang, S.; Wu, M.; Jia, Z. Matrix Inequality; Science Press: Beijing, China, 2006.

(C) 2019 by the authors. Licensee MDPI, Basel, Switzerland. This article is an open access article distributed under the terms and conditions of the Creative Commons Attribution (CC BY) license (http://creativecommons.org/licenses/by/4.0/). 